Portland State University

PDXScholar

1989

\title{
The Longterm Psychosocial Impacts of Caregiving on the Caregivers of Persons with Stroke
}

Alice Updike Scannell

Portland State University

Follow this and additional works at: https://pdxscholar.library.pdx.edu/open_access_etds

Part of the Social Policy Commons, and the Urban Studies Commons Let us know how access to this document benefits you.

\section{Recommended Citation}

Scannell, Alice Updike, "The Longterm Psychosocial Impacts of Caregiving on the Caregivers of Persons with Stroke" (1989). Dissertations and Theses. Paper 1312.

https://doi.org/10.15760/etd.1311

This Dissertation is brought to you for free and open access. It has been accepted for inclusion in Dissertations and Theses by an authorized administrator of PDXScholar. Please contact us if we can make this document more accessible: pdxscholar@pdx.edu. 
THE LONGTERM PSYCHOSOCIAL IMPACTS OF CAREGIVING ON THE CAREGIVERS OF PERSONS WITH STROKE

\title{
by
}

\begin{abstract}
ALICE UPDIKE SCANNELL
DOCTOR OF PHILOSOPHY

in

URBAN STUDIES
\end{abstract}

Portland State University

(C) 1989 
TO THE OFFICE OF GRADUATE STUDIES:

The members of the Committee approve the dissertation of Alice Updike Scannell presented December 7, 1988.

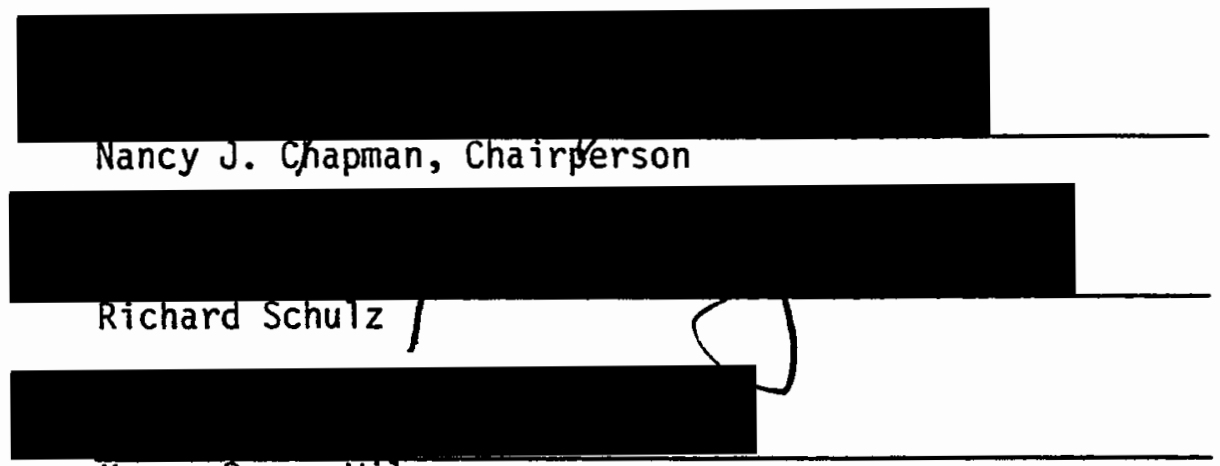

Keren Brown Wilson

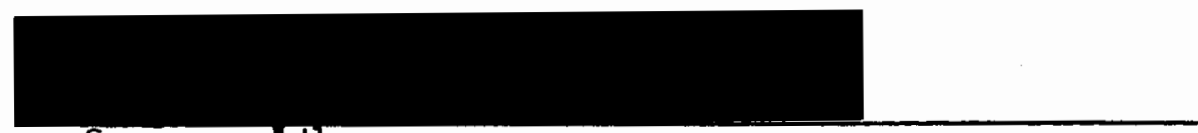

Seymour Adler

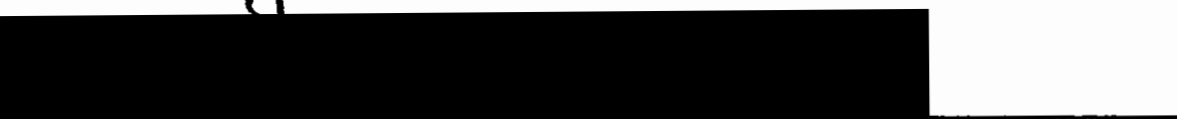

Cathleen L. Smith

APPROVED :

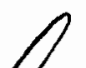

Nohad A. Toutan, yean, Schook of Urban and Public Affairs

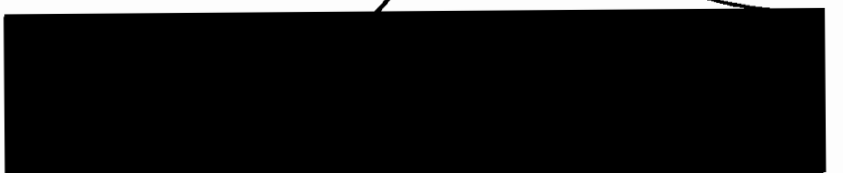

Bernard Ross, Vice Provost for Graduate Studies 


\section{ACKNOWLEDGEMENTS}

The final product of any research project is often possible only because of the assistance and support of many people at different steps throughout the process. I especially want to thank the members of my committee: Dr. Nancy J. Chapman, Chairperson, Dr. Richard Schulz, Dr. Seymour Adler, Dr. Keren Brown Wilson, and Dr. Cathleen L. Smith for their helpful suggestions and timely reading of the drafts.

In addition, the following people were among those who were very important to this project: Dr. Judy Rau, who designed the original stroke study; Charlene Rhyne, project manager of the original stroke study; Christine Curnow, research assistant at the University of Pittsburgh who assisted with data collection and data entry; Wes Brenner and Dave Sato of Portland State University's Computing Services; and Dr. David Morgan, of the Institute on Aging and School of Urban and Public Affairs at Portland State University, who assisted in the working out of many analytical issues and generally helped me to keep my eye on the light at the end of the tunnel instead of the dark within it.

I acknowledge also the grants from the AARP Andrus Foundation and the National Institute on Aging (AGO 6243) which provided funding for the original study.

Finally, I am grateful to my sons Stephen and Andrew and to my husband John for their support and good cheer throughout the project. 


\section{DEDICATION}

This dissertation is dedicated to my mother, Mary Alice Riddick Updike, who exemplifies the classic model of the family caregiver. In the mid-1940's my mother gave up a teaching position so that she could be at home when my paternal grandmother, who was paralyzed with Parkinson's disease, and her nurse came to live with us, expanding our household to nine persons. Several years after my paternal grandmother's death at age 87, my mother's own mother came to live with us for several years until her death from a stroke at age 86 . Now, at age 91, even after experiencing two strokes herself within the past decade, my mother continues to provide both emotional and financial support to her children and grandchildren.

My mother has been very interested in the process of my work in gerontology and in the writing of this dissertation. Her support and interest have been invaluable to me, and with this dedication I honor her. 
TABLE OF CONTENTS

PAGE

ACKNOWLEDGEMENTS $\ldots \ldots \ldots \ldots \ldots \ldots \ldots \ldots \ldots \ldots \ldots \ldots \ldots \ldots \ldots$ i $i$

DEDICATION ................................. iv

LIST OF TABLES $\ldots \ldots \ldots \ldots \ldots \ldots \ldots \ldots \ldots \ldots \ldots \ldots \ldots \ldots \ldots \ldots \ldots$

LIST OF FIGURES $\ldots \ldots \ldots \ldots \ldots \ldots \ldots \ldots \ldots \ldots \ldots \ldots \ldots \ldots \ldots$ ix

CHAPTER

I INTRODUCTION AND LITERATURE REVIEW ............... 1

II DESIGN, METHODOLOGY, AND RESULTS FROM

THE ORIGINAL STUDY .......................... 24

II I CONCEPTUAL FRAMEWORK FOR THE T4 STUDY ............ 33

IV METHODOLOGY $\ldots \ldots \ldots \ldots \ldots \ldots \ldots \ldots \ldots \ldots \ldots \ldots \ldots \ldots \ldots \ldots \ldots \ldots$

v DESCRIPTIVE ANALYSIS $\ldots \ldots \ldots \ldots \ldots \ldots \ldots \ldots \ldots \ldots \ldots \ldots \ldots . \ldots \ldots$

Demographic Characteristics .................. 62

Characteristics of the Caregiver's

Social Support System ..................... 72

Caregiver Stress and Burden ..................... 79

Responses to Stress: Coping and Adjustment ........ 81

Psychosocial Outcome Measures ................. 82

VI FINDINGS $\ldots \ldots \ldots \ldots \ldots \ldots \ldots \ldots \ldots \ldots \ldots \ldots \ldots \ldots \ldots \ldots \ldots$

Characteristics of the Caregiver ............ 87

Results of Multiple Regression Analyses ......... 111

Hypotheses Supported and Not Supported ......... 118 
VII CHANGES IN SELECTED VARIABLES BETWEEN T1,

T2, T3, AND T4 ........................... 123

Changes in Depressive Symptomatology, Positive and Negative Well-being, and

Perceived Burden Over Time ...................... 123

Changes in Selected Items of Perceived

Burden Over Time ........................... 130

Changes in the Mode of Transportation

for the Person with Stroke .................... 133

VIII DISCUSSION AND IMPLICATIONS $\ldots \ldots \ldots \ldots \ldots \ldots \ldots \ldots \ldots \ldots \ldots$

Areas for Future Research .................. 157

Implications of the Study ................. 160

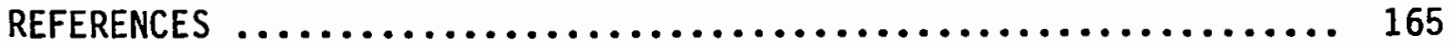

APPENDIX

A Recruitment Letter ......................... 176

B Informed Consent .......................... 178

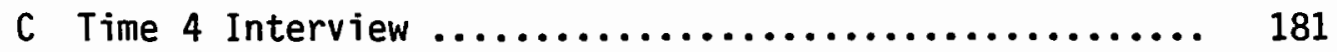




\section{LIST OF TABLES}

TABLE

PAGE

I Reasons for Attrition between Time 1 and Time $4 \ldots \ldots . .44$

II Means, Standard Deviations and T-test Results for Subjects Participating in Time 1 versus Participants in Both $\mathrm{T} 1$ and $\mathrm{T} 4$.................. 59

III General Demographic Data for All Caregivers ........... 63

IV Percentage of Current Caregivers Performing Specific Tasks for Person with Stroke ............. 70

$V$ Changes Since Stroke in Personality/Behavior of Care-recipient at T4....................... 78

VI Pairwise Correlations of Variables in the Model ....... 90

VII T4 Correlations of Individual Personality/Behavior Changes in the Stroke Patient with Caregiver Scores for CES-D, Burden and Adjustment ............. 101

VIII T4 Correlations of Individual Items on the Caregiver Burden Scale with Caregiver Scores for CES-D and Adjustment ......................... 105

IX T4 Correlations of Caregiver Adjustment with Stroke Variables, Perceived Stress, and Outcomes ...... 110

$X \quad$ Hierarchical Regression for CES-D at T4 ........... 115

XI Hierarchical Regression for NEGWB at T4 ............ 117

XII Hierarchical Regression for POSWB at T4 ............. 119

XIII Hypotheses Supported and Not Supported by the Study ................................. 120

XIV Means of CES-D, NEGWB, POSWB, and Burden Scores for T4 Sample of Current Caregivers Over Time ........... 124 
XV Percent of T4 Sample With Symptoms Indicating Clinical Depression Levels on the CES-D Measure Over Time ...... 125

XVI Percent of T4 Sample Indicating Negative Changes in Personality/Behavior Characteristics Since the Stroke in the Person With Stroke Over Time .......... 128

XVII Percent of T4 Sample Indicating Experience of Burden as 'Often' or 'Almost Always' on Selected Items of the Caregiver Burden Score Over Time

XVIII Percent of T4 Sample Indicating Stroke Patients' Ability to Provide Own Transportation

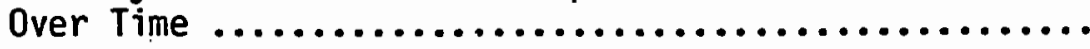




\section{LIST OF FIGURES}

$\begin{array}{ll}\text { FIGURE PAGE } & \text { PE }\end{array}$

1. The Mode1 $\ldots \ldots \ldots \ldots \ldots \ldots \ldots \ldots \ldots \ldots \ldots \ldots \ldots \ldots \ldots . \ldots \ldots$

2. Measurement of Variables at Each Time Period ........... 47

3. Depression by level of strain with satisfaction with quality of social contact ................... 99

4. Depression by level of perceived burden with satisfaction with quality of social contact ........... 99 
AN ABSTRACT OF THE DISSERTATION OF Alice Updike Scannell for the Doctor of Philosophy in Urban Studies presented December 7, 1988.

Title: The Longterm Psychosocial Impacts of Caregiving on the Caregivers of Persons with Stroke.

APPROVED BY MEMBERS OF THE DISSERTATION COMMITTEE:

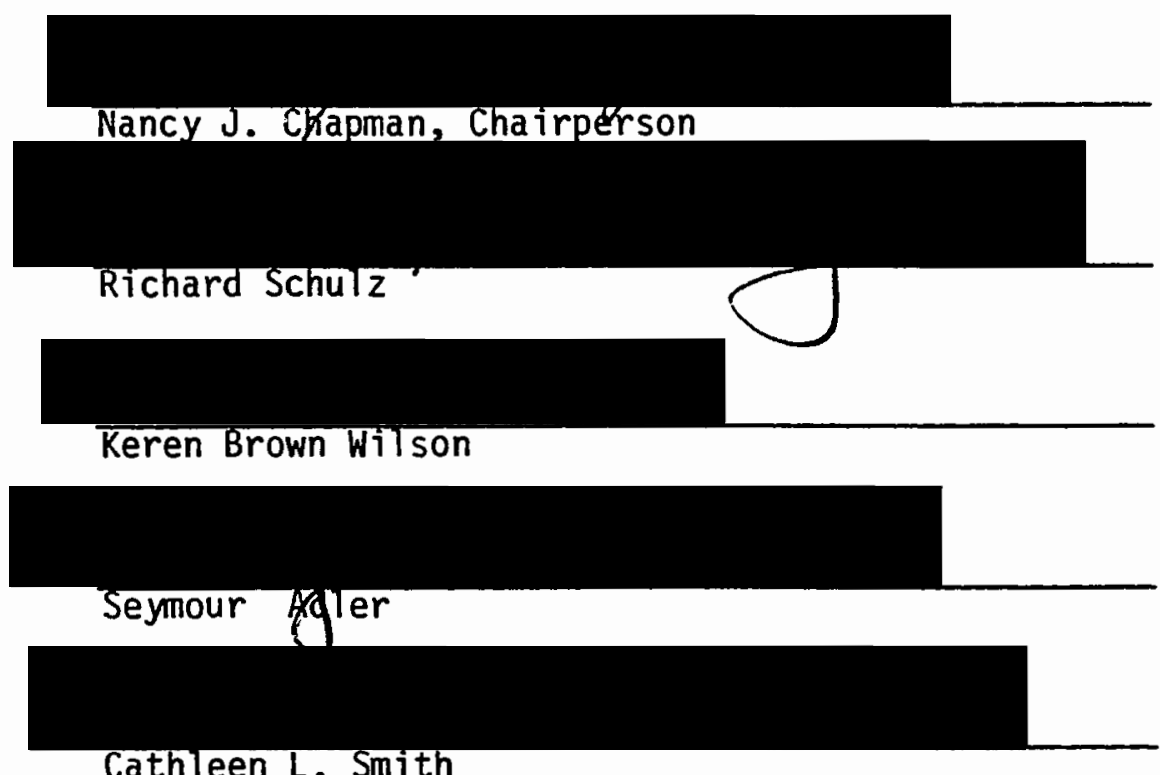

Families are vital resources for the provision of long-term care services for the frail elderly and other functionally impaired persons whose capacity for self-care is restricted. Although the attention of much recent research has focused on family caregivers, most studies are either cross-sectional, have small samples, or include a variety of caregiving situations.

The study reported in this dissertation is a Time 4 (T4) followup interview of the caregivers of persons who experienced a first 
stroke between 2 to 4 years (mean=36 months) prior to the T4 interview. The first wave of data collection (T1) occurred within two months after the stroke. The second and third waves occurred six and twelve months, respectively, after the first interview. The caregivers were identified by the person with stroke as being the person closest to $\mathrm{him} / \mathrm{her}$ who would be responsible for care after the stroke. Ninety-three respondents participated at T4.

Data were gathered at all four interviews using reliable and valid measures for depressive symptomatology (CES-D; Radloff, 1977), psychological well-being (IPWB; Berkman, 1971), and caregiver burden (Zarit, 1980). The contribution of social support to caregiver wellbeing was also investigated. Additional areas of investigation at T4 included coping strategies (F-Copes; McCubbin, Larsen, and 01son, 1981), caregiver adjustment, and the respondents' perception of themselves as "caregivers".

The mean scores of depressive symptomatology, perceived burden, negative well-being, and positive well-being did not change significantly over the four points in time. However, the percentage of the sample having CES-D levels of 16 and above (indicating potential diagnosis of clinical depression) decreased by ten percent between $\mathrm{T} 1$ and $\mathrm{T} 4$. About ten percent of the respondents who were at risk for clinical depression at T4 reported high levels of depressive symptoms at all four interviews.

Sixty-two percent of the respondents thought of themselves as "caregivers" and thirty-eight percent did not. Those who thought of themselves as caregivers were significantly more likely to report high 
levels of depressive symptoms, to experience high levels of strain and caregiver burden, and to be caring for persons who were more severely impaired by the stroke than those who did not.

The experience of burden and the outcomes of depressive symptomatology were significantly associated with both the functional capacity of the person with stroke and with an index of stroke severity comprised of communication impairments and negative personality/behavior changes since the stroke. However, positive well-being and negative well-being were significantly associated only with the stroke severity index and not with the measure of functional capacity. This finding suggests that the residual personality/behavior changes which often result from stroke have longterm negative effects on the caregiver and may be very important in measuring overall stroke severity and the patient's recovery from stroke.

There were no significant gender differences in depressive symptoms, positive well-being, negative well-being, or caregiver burden, nor were there significant differences in outcomes between caregivers who were living with the person with stroke and those who were not. However, non-spousal caregivers reported significantly higher levels levels of depressive symptomatology than spousal caregivers $(p<.01)$.

The results of multiple regression analysis showed that caregiver characteristics contributed more to the variance in depressive symptoms and psychological well-being than did characteristics of the stroke. The significant predictor of 
depressive symptomatology was the caregiver's subjective health status $(p<.01)$. Significant predictors of negative well-being were perceived burden $(p<.01)$ and low satisfaction with social support $(p<.05)$. The significant predictor of positive well-being was the use of coping strategies $(p<.05)$.

The findings from this study have implications for stroke management programs, caregiver intervention planning, and health care pol icy. 


\title{
CHAPTER I
}

\section{INTRODUCTION AND LITERATURE REVIEW}

\author{
INTRODUCTION
}

Caregivers of functionally impaired persons of all ages have been the subject of recent research. Public policy has increasingly focused on ways to minimize the use of nursing homes and other longterm care facilities in favor of less expensive alternatives within the community. Informal caregivers--family, friends, neighbors, and community volunteers--are vital resources in the system of providing community-based care for adults whose capacity for self-care is restricted. In order to support family caregivers in providing longterm care for the impaired elderly, several states have enacted tax provisions for family members who provide care in their own homes for elderly relatives. Other states are engaged in studies to determine the feasibility of providing direct payments to families who provide care for elderly relatives (Arling and McAuley, 1983).

Jones and Vetter (1984) report that the World Health Organization Congress on Ageing strongly reinforced the concept that the elderly should be supported to live in the community, and furthermore to live in the community with their families. Such a policy has implications not only for the elderly or for community- 
based social and medical services, but also for the families who provide support for frail and disabled elderly.

Studies of the impact of caregiving on the caregiver are important for the development of both policy and practice in long-term care. Caregiving over a period of time may result in mental and physical health problems for the caregiver due to fatigue, stress, physical strain, and confinement (Cantor, 1983; Fitting, Rabins, Lucas and Eastham, 1986; Fengler and Goodrich, 1979). In addition, institutional placement of disabled or frail elderly has been shown to be more associated with the breakdown of the health and/or emotional resources of family caregivers than with the level of disability of the care-recipient (Zarit, Todd, \& Zarit, 1986; Morycz, 1985).

Within the past few years hospitals and social agencies have developed caregiver programs designed to provide information, support, and respite care for persons who are providing help for functionaliy and neurologically impaired individuals. These programs have been based on the assumption that caregiving is a role which, without intervention, ultimately drains the caregiver. "Caregivers need care too" has become a popular slogan seen even on bumper stickers. Current attention is being given to the effects of caring for elderly relatives on the work performance of employed caregivers (Enright and Friss, 1987).

Some studies have indicated that caregivers of functionally impaired adults are actually "hidden patients" whose own needs for support and training should be considered at the outset of planning for the patient's care (Fengler and Goodrich, 1979). However, very few 
longitudinal studies are available which provide information about factors associated with adaptation to caregiving over time. Longitudinal studies are needed which consistently measure caregiver and care-recipient characteristics over a period of several years. Data from such longitudinal studies would contribute valuable information for use in long-term care policy development as well as for the planning of caregiver interventions and support programs.

The study presented in this dissertation is a longitudinal caregiver study. It was designed to add a fourth wave of data collection to a longitudinal study which had examined the role of social networks and social support in the lives of stroke patients and their primary support person (caregiver) during the year following a first stroke (Schulz and Rau, 1984). The study reported in this dissertation is focused on data gathered from the caregivers only and not the persons with stroke. The design and data collection procedures of the original longitudinal study, along with major findings from the three waves of data collection contained in the original study, are presented in Chapter II. In addition to gathering data on psychosocial outcomes with instruments used in the first three interviews, the study reported in this dissertation included additional measures of strain, coping strategies, and perceived adjustment to the stroke.

The impetus for selecting the topic of the long-term psychosocial impacts of caregiving on the primary support persons of stroke victims came from my experience as a research assistant on the original project. I had interviewed many respondents right after the 
stroke, some at the 6 month point after the first interview, and others at the one-year follow-up interview. Many of the primary support persons seemed to be under high stress and they expressed feelings of isolation and despair at the one-year interview. This seemed to occur especially for the subjects who were caring for persons who continued to show residual stroke-related changes in personality/behavior characteristics. I had expected that the support persons would have adapted well to the stroke during the first year, and was intrigued by evidence that this was not necessarily the case. Therefore, I wanted to design a study which would investigate some of the specific issues in, experiences of, and responses to caregiving, and look at the psychosocial impacts of the stroke experience on the caregiver beyond the second year after the stroke.

In order to study the relationship of caregiving and psychological well-being, and to develop a theoretical basis for the model presented in this dissertation, a literature review was undertaken to identify the important variables which could be tested as predictive of positive or negative outcomes, and those which could be predicted as mediating between the stresses of caregiving and the outcomes of depressive symptomatology and psychological well-being.

The following literature review is divided into five sections. Patterns of family caregiving are discussed first, with some attention given to cross-national comparisons in family caregiving. Then a review of the literature on the specific characteristics of providing care for a person who has experienced a stroke is presented. The third section of the review examines the literature relating to 
caregiver burden and stress; the fourth section discusses the buffering effect hypothesis as it relates to factors which mediate between stress and psychological well-being outcomes. The final section presents some important policy issues raised by family caregiving.

The theoretical model which is based on the literature will be presented in Chapter III, and tested in the design and analys is which are described in the remaining chapters.

\section{FAMILY CAREGIVING}

Although articles continue to appear in the popular press which perpetuate the myth that Americans have abandoned their elderly members by placing ("warehousing") them in institutions (Szulc, 1988) empirical evidence indicates that families are actually caring for functionally disabled members at least as much or even more than ever before (Shanas, 1979; Stone, 1987; Stephens and Christianson, 1986). The involvement of families in providing support and care for impaired elderly has been clearly documented in the literature (Shanas, 1979a, 1979b; Brody, 1985; Stephens and Christianson, 1986). However, that care does not generally come without emotional costs to the caregiver. Descriptive studies generally find high levels of strain among family caregivers (Cantor, 1980; Brody, 1981; Brody et al., 1986; Johnson, 1983), and the erosion of family emotional resources has been found to be a critical variable in the decision to institutionalize an impaired older person (Morycz, 1985; Zarit et al., 1986). 
Caregivers provide care and support to elderly or disabled members in several different settings. In order to design caregiver programs to meet specific needs, it is important to know if there are significant differences for long-term caregiver outcomes between the settings in which care is most often provided. Many caregivers live with the person who needs the assistance. This type of caregiving is often defined as 'intrahousehold' caregiving, and has been found in some studies to have a greater negative effect on the caregiver than other types (Soldo and Myllyluoma, 1983; Stephens and Christianson, 1986). 'Interhousehold' caregiving is the term given to situations in which the care-recipient lives in a separate household from the caregiver. A common type of interhousehold caregiving is that provided by an adult child who lives in her/his own home to a parent or parents who live in their own dwelling. Sometimes other relatives or even friends will be caregivers for an elderly person who lives alone. The third common setting for caregiving is that in which the care-recipient lives in a long-term care facility of some type such as an adult foster home, a congregate or assisted living setting, or a nursing home. Caregivers are often as much involved in a caregiving role after placement as before, and though they may be providing fewer instrumental tasks for the care-recipient there appears to be little relief from the experience of burden and emotional responsibility (Pratt, Wright, \& Schma11, 1987; Zarit et al., 1986).

The largest burden of caregiving generally falls to one individual, especially if that person lives with the care-recipient (Soldo and Myllyluoma, 1983; Stephens and Christianson, 1986). This 
finding is supported by the work of Johnson and Catalano (1983), who found in a longitudinal study of family supports to the impaired elderly that support is extended most often by one family member at any given time. Jones and Vetter (1984) found in their study of 256 family caregivers in wales that caring for a disabled elder does not involve a large network of informal or formal assistants, particularly if the caregiver lives with the care-recipient.

Empirical evidence is inconclusive about the role which family relationship plays in the psychosocial outcomes of caregiving. Studies which have compared the effects of caregiving on spouses versus adult children (Benedict, Lovett and Gallagher, 1986; VanWinkle, 1987) have found few significant differences in the effects of caregiving on levels of burden, morale, or depressive symptomatology. However, Benedict et al. (1987) report that adult children were significantly more likely than spouses to report a higher number of troublesome behaviors from the care-recipient, and a higher level of distress due to the care-recipient's troublesome behaviors. Van Winkle (1987) studied depressive symptomatology (using the Center for Epidemiological Studies Depression scale--CES-D) in a nonrandom sample of 31 spouses and 28 adult children of patients with dementia. Although the differences in the mean scores of the CES-D between spouses and adult children were not significant at the $p<.05$ level, a higher percentage of both spouses and adult children who live with the care-recipient and spouses who had placed the carerecipient in a nursing home had higher prevalence rates of depressive symptomatology than is found in the general population. 
Jones and Vetter (1984) found that thirty-six percent of their sample of 256 family caregivers scored as pathological (13\%) or borderline pathological (23\%) on a validated anxiety scale. In a comparison of spouses and daughters, the daughters were significantly higher in anxiety levels. However, spouses and daughters showed similar morbidity in measures of depression. Daughters also reported significantly more stress than spouses, with $17 \%$ of the daughters finding that looking after the dependent parent caused 'a lot of stress' and another $14 \%$ indicating that they found their caregiving role to be 'unbearable' (Jones and Vetter, 1984).

Johnson and Catalano (1983) found that spouses were more likely than adult children to provide more comprehensive care over time with a minimum of stress or ambivalence. Adult children, on the other hand, were more likely to turn to formal supports or seek the assistance of other family members.

Cicirelli (1983) reports that adult children often mention feeling stressed even when they are providing little or no tangible help to an aging parent. In his study of the role of adult children in helping elderly parents (Cicirelli, 1981) it was found that personal strain and negative feelings were more strongly related to perceived parental dependency than to actual amount of help provided to parents. He postulates the operation of a construct of "filial anxiety" in these situations, in that adult children experience anxiety when contemplating or anticipating the possibility of providing help to parents and worry about their own ability to deal with the impending burdens. They may feel a need to monitor their 
parents' well-being more carefully, creating increased worry, anxiety and stress. Filial anxiety was found to be related to perceptions of the parent as declining in mobility, cognitive functioning, and general social adjustment but not to parental illness or amount of help given (Cicirelli, 1983).

Studies have also examined gender differences in the impacts of the caregiving role (Zarit et al., 1986; Horowitz, 1985). Zarit et a1. (1986) examined gender differences in spouses of dementia patients, and found that husbands experienced significantly less burden than wives at the first interview, but that the burden experienced by wives had significantly decreased by the follow-up interview two years later. No significant gender differences in burden were found between husbands and wives at the two year followup. The authors postulate that the differences in burden scores may have been due to the husbands' ability to adopt an instrumental approach to daily problems at the beginning, but that the wives needed more time to develop emotional distance from the problems in order to adopt more instrumental coping strategies. In both cases, however, Zarit et al. (1986) note that the caregiver's ability to tolerate problem behaviors increased as the patient's disease progressed.

A cross-sectional study by Horowitz (1985) examined gender differences in a sample of 131 adult children who had been identified as the primary caregiving relative of an elderly parent. In addition to examining differences in types and extent of assistance provided, the investigation looked at differences in perceptions of the social and emotional consequences of caregiving. Findings indicated that sons 
have less stressfut caregiving experiences than daughters even though they are as likely to have a parent living with them as are daughters and are as likely to provide emotional support, financial aid and linkage services as are the daughters. Although all the parents in Horowitz' (1985) study were utilizing some type of formal services, daughters were still more likely to be providing "hands-on" services to the parent than were sons.

The availability and utilization of social support (especially the informal support system of family, friends, and neighbors) has long been thought to be associated with positive outcomes for caregivers. Johnson and Catalano (1983) compared the difference between caregivers whose morale improved versus those whose morale remained stable or declined (all in conditions of decreased functioning of the care-recipient) and found that sixty-four percent of the caregivers whose morale had improved over a period of eight months were using some type of formal supports. Kessler and McLeod (1985) suggest that the perceived availability of support is more important for caregiver well-being than actual use of support. Ward et a1. (1987) report finding that having a reciprocal confiding relationship in one's social network was important for positive caregiver outcomes. George (1987) reports that the relationships between caregiver well-being and perceptions of the adequacy of social support are stronger than (and independent of) the relationships between objective levels of social support and carevgiver well-being. 
Studies on specific experiences with life crises such as widowhood (e.g. Vachon et al. 1982a, 1982b) or unemployment (Gore, 1978) have found social support to be an important predictor of emotional adjustment. However, as noted by Kessler et al. (1985), there is little consensus on how to define successful adjustment. Adjustment may be thought to reflect mastery of the crisis situation, but on the other hand emotional distress may be the impetus for developing effective coping efforts.

Gibson (1984), in an extensive review of family caregiving from a cross-national perspective, states that research on family helping patterns in western nations, in the more traditional societies, and in Eastern European countries are very similar. In all countries many chronically $i 11$ and functionally impaired elderly are being cared for by family and friends. Evidence is strong that the elderly prefer to be cared for by family if they cannot do the tasks themselves. The major types of assistance provided by informal providers in most developed nations are personal care, homemaker services, and transportation (Gibson, 1984). Evidence is lacking about family caregiver's preference to be the major provider of assistance for the elderly, although some studies show that there is a feeling of obligation expressed by both spouses and adult children (Borgatta and Montgomery, 1987).

Two major issues in long-term care policy are eligibility for services and funding of those services. Although a few countries have included provision for caregiver respite and other community based services in systems of universal access (Gibson, 1984), many developed 
countries are struggling with the costs of community-based long-term care services (Gibson, 1984).

THE EXPERIENCE OF CAREGIVERS OF PERSONS WITH STROKE

Although stroke is the third leading cause of death in this country (following heart disease and cancer; National Center for Health Statistics, 1986), it is of primary interest in this study because it is also a leading cause of chronic functional disability (Dyken, 1987). Men have a greater risk of experiencing stroke than women (Dyken, 1987). More than $66 \%$ of persons with stroke from a national sample of community-residing stroke patients had residual chronic disabilities (Baum and Robins, 1981; Baum, 1982). Most persons with stroke-related disabilities will receive care at home from a spouse or other relatives (Ahlsio, Britton, Murray, Theorell, 1984; DeJong and Branch, 1982; Holbrook, 1982).

Persons who have experienced a stroke often have long-term residual effects of severe communication difficulties and/or paralysis and loss of sensation on one side of the body. Stroke is generally accompanied by cognitive, behavioral, and/or personality changes (Binder, 1983; Lezak, 1978a; Stein, Hier, and Caplan, 1985). Anxiety, frustration, and depression are common emotional problems that remain with persons who have had a stroke long after discharge from care (Binder, 1983). Other common problems are overdependence on others, impatience, irritability, impulsivity, poor social perception, perplexity, distractibility, and fatigue (Binder, 1983; Lezak, 1978b; Stein et al., 1985). Even mildly impaired stroke patients.will 
experience long-term perplexity, distractibility, and fatigue (Lezak, 1978b).

Labi, Phillips, and Gresham (1980) analyzed social reintegration in a sample of long-term survivors of stroke, and found that even within the group of subjects who could walk independently and had not experienced any falls about half decreased their socialization outside the home after the stroke. Neither age, physical impairment, nor specific neurological defects were significantly associated with the decrease in socialization. Rau (1986) suggests that a possible explanation for these results could be what Hyman (1971) has identified as the "stigma of stroke", defined as the belief "that others think less of them, avoid them, or feel uneasy with them because of their illness or disability" (Hyman, 1971, p. 132).

The subtle long-term residual effects mentioned above, as well as the more visible impairments of paralysis or aphasia (loss of speech) could be expected to have a strong negative impact on the well-being of the caregivers of persons who have experienced a stroke.

Studies which have concentrated on the impact of stroke on caregivers and families of the person with stroke generally have found that psychosocial adjustments to the stroke are difficult for caregivers to make (Belcher, Clowers, and Cabanayan, 1978; Cohen, 1978; Holbrook, 1982; Coughlan and Humphrey, 1982). Caregivers often report personality changes in the person with stroke which include irritability, loss of self-control, decreased tolerance for frustration, impatience, self-centeredness and decreased initiative 
(Cohen, 1978; Coughlan and Humphrey, 1982; Brocklehurst, Morris, Andrew, Richards, and Laycock, 1981). In a study of 284 caregivers of brain-impaired adults Enright and Friss (1987) report that caregivers of relatives who were either stroke victims or had a brain tumor reported higher levels of stress than caregivers of relatives suffering from dementia.

Not only can caring for a person who has experienced stroke have a negative emotional impact on the caregiver, it often has negative impacts on the physical health of the caregiver as well. Brocklehurst et al. (1981) report that the health of 97 primary caregivers of new stroke patients deteriorated a great deal in the first year after the stroke. Reported deterioration of caregiver health tripled in the time between the first interview (approximately one month after the stroke) and the one year post-stroke interview.

Studies with samples of stroke patient caregivers have found few significant relationships between the level of physical impairment or functional disability of the stroke patient and negative impacts on the caregivers (Kinsella and Duffy, 1979; Silliman, Fletcher, Earp, and Wagner, 1986). However, although silliman et al. (1986) found no significant relationship between the stroke patients' disability and declines in the caregivers' physical health, they did find a significant association between the functional capacity of the stroke patient and detrimental effects in the caregivers' emotional and social health.

The site of lesion, i.e. the area in the brain which contains the tissue damaged by the stroke, is another subject of investigation 
in studies concerned with the effects of stroke on both the patient and the caregiver. Left hemisphere strokes affect the speech and language functions of the brain and may cause moderate to severe aphasia (loss of speech and verbal functions). Left hemisphere strokes may also result in moderate to severe paralys is of the right side of the body. Right hemisphere strokes affect the left side of the body, and may also affect the ability to recognize or verbalize emotion (Whitney, 1987). Strokes may also occur elsewhere, such as in the brain stem, on the brain's surface or in the spinal column. These strokes are extremely serious and often result in death or massive disability (Caplan, 1987).

Although the evidence is not clear about the relationship of site of lesion to long-term impacts on the caregiver of the person with stroke, some investigators have found that caregivers are significantly more likely to report negative impacts on their lives if the person with stroke had experienced a right hemisphere stroke rather than other types of stroke (Coughlan and Humphrey, 1982). These effects may be due to the loss of emotional affect which often occurs with a right hemisphere stroke and which may prevent the stroke patient from responding to the caregiver in a reciprocally nurturing or supportive manner.

Some studies have examined the role of interaction between the person with stroke and the caregiver. Henley (1985) found that stroke patients were significantly more likely to have positive rehabilitation outcomes if they had a partner and Whitney (1987) suggests that the partner functions as a buffer between the negative 
behavior/attitudes of the stroke patient and the rehabilitation process. A partner also provides motivation for increased activity. The negative behavior/attitude factor is important in the lives of both the stroke patient and the caregiver, for it has been found to be one of the predictors of poor prognosis for stroke recovery regardless of whether the observed behavior/attitude was present before the stroke or is a consequence of the stroke (Whitney, 1987).

Silverstone and Horowitz (1987) compare the issues of caregiving for stroke patients versus caregiving for the chronically impaired or frail elderly, and indicate that there are several important differences. One of these is the sudden onset of stroke versus the gradual onset of other types of physical disabilities. (such as arthritis or heart disease) or mental impairments (such as Alzheimer's disease). In gradual onset diseases the gradual increase in the frait elder's need for support may allow families to adapt slowly to the caregiving role. In contrast, the effects of stroke are usually presented suddenly and dramatically to the family, and a severe family crisis generally occurs when a relatively healthy and previously selfsufficient family member becomes incapacitated in a very brief period of time.

In addition, Silverstone and Horowitz (1987) point out that the prospective payment plan enacted by Medicare policy often leads, to discharge from the hospital before the end of the acute phase of the stroke, and may require that the family caregivers be able to assist with providing skilled care as well as rehabilitative care to the stroke patient. 
Two other differences between caring for a stroke patient and other types of chronically ill or frail elderly lie in 1) the potential for recovery from stroke and 2) the communication and emotional problems that are peculiar to stroke. Most other types of chronic illness or frailty can at best be expected to stabilize and are more likely to have a prognosis of gradual decline in functioning. Stroke patients can recover from the effects of the stroke. Knowing that there is a possibility of recovery but not seeing much recovery over time can lead to pessimism by both the stroke patient and the caregivers about the value of rehabilitation. On the other hand, it can also lead to romantic notions about the potential for recovery and to the expectation that rehabilitation should lead to complete recovery (Silverstone and Horowitz, 1987). Caregivers may blame the stroke patient for "not trying harder" to do things for him/herself. The stroke patient's communication difficulties and emotional problems can cause confusion and frustration for family members and have been found to be a prime source of stress for caregivers. In addition to not knowing how to communicate their own emotional support to the person with stroke, caregivers are deprived of the emotional support previously received from the stroke patient (Silverstone and Horowitz, 1987).

CAREGIVER STRESS, BURDEN, DEPRESSIVE SYMPTOMATOLOGY, AND PSYCHOLOGICAL WELL-BEING

As we have seen, the literature on family caregiving points to consistent findings of stress, strain, and burden among family 
caregivers (Cantor, 1980; Brody, 1981; Brody et al., 1986; Johnson, 1983). Caregiving stresses have been found to be significantly associated with high depressive symptomatology (Van Winkle, 1987) and clinical depression (Jones and Vetter, 1984). Coppel, Burton, Becker, and Fiore (1985) report that $87 \%$ of their sample of 68 caregivers for Alzheimer's victims were either currently depressed or had met criteria for depression at some point during the caregiving process. Gallagher, Wrabetz, Lovett, Del Maestro, \& Rose (in press) found that between 40 and 50 percent of their sample of 115 caregivers of frail elders were currently clinically depressed. Untreated depression does not remit solely with the passage of time (Thompson, Gallagher, and Breckenridge, 1987; Lehman, Wortman, \& Adams, 1987). Identification of caregivers who are at risk for depression is therefore an important function of research on the long-term psychosocial impacts of caregiving.

\section{THE BUFFERING HYPOTHESIS: COPING AND SOCIAL SUPPORT AS MEDIATORS OF STRESS}

The buffering hypothesis (Thoits, 1982) suggests that certain factors may mediate between the effects of a stressful life event and negative psychosocial outcomes. Among the important factors thought to act as mediators under conditions of high stress are coping strategies and social support. Folkman and Lazarus (1988) understand the relationship between emotion and coping in stressful encounters as being bi-directional, with each application of a coping strategy directly affecting the emotional response and vice versa. When a 
person appraises a situation as stressful or non-stressful both the appraisal and its attendant emotions influence the coping processes. The selected coping strategy will in turn change the personenvironment relationship. Then the altered condition is reappraised, leading to a change in the emotion. Within that dynamic context coping is seen as a mediator of the emotion response.

The relationship between coping and responsiveness to 1 ife stress has been studied in a variety of ways and with varied results. Kessler, Price, \& Wortman (1985), in a review of the coping literature, conclude that little progress has yet been made in developing objective, reliable, and valid ways of capturing the coping process.

The studies which have examined the role of coping strategies in facilitating adjustment to specific life crises such as loss of a child (Videka-Sherman 1982), rape (Burgess and Holmstrom 1979), or divorce (Berman and Turk 1981) indicate that coping processes may play at least a modest role in ameliorating the impact of life stressors (Kessler et al., 1985).

Among the coping strategies employed by victims of life crises, turning to professionals for help is rare. Veroff et al. (1981) found that victims of life crises are much more likely to turn to informal support systems, such as family, friends, and neighbors than. to professionals. Coping and social support intersect conceptually, and Thoits (1983) suggests that social support may actually be conceptualized as "coping assistance". 
In a study which compared the effects of coping on the emotional responses of young adults (mean age $=40.0$ ) and older adults (mean age $=68.6$ ) Folkman and Lazarus (1988) found some notable differences between the age groups in the effects of coping on emotion. One of these effects was that positive reappraisal (a cognitive process of seeing some positive aspects of negative events) was associated with a decrease in distress and an increase in positive feeling in the younger group and with a worsened emotion state of more worry or fear in the older group. Seeking social support was associated with increased positive emotions in the older group, but showed no association with positive emotions in the younger group. These differences have potential implications for the development of caregiver education and intervention strategies.

Kessler and McLeod (1985) conclude from a review of the literature on the stress-buffering effects of social support that evidence indicates that two types of social support were strongest in diminishing the impact of life stress on mental distress. These were identified as 1) emotional support and 2) the perception of access to broad-based support that could be mobilized if needed as opposed to actual amounts of support received.

\section{FAMILY CAREGIVING: POLICY IMPLICATIONS}

Studies which have attempted to determine policy-relevant information about the effects of caregiving on the long-term health and well-being of family caregivers include those of Morycz (1985), 
Poulshock and Deimling (1984), Zarit et al. (1986), George and Gwyther (1986), Soldo and Myllyluoma (1985) and Pratt, Wright and Schmall (1987)

Understanding the effects of caregiving on an individual's mental and physical health provides information for social service planning and delivery (Lowy, 1983; Hooyman and Gonyea, 1985) and for the planning and implementation of preventive programs oriented toward maintaining caregivers' health and well-being. Pratt et al. (1987) found that caregiver burden was related to several coping strategies. Acquiring support from the extended family was associated with lower levels of burden for caregivers of community-dwelling Alzheimer's patients. Because studies show that caregivers often have difficulty identifying the needs that others might be able to meet, and in identifying and utilizing other family members as sources of support (Springer and Brubaker, 1984), Pratt et al. (1987) suggest that professionals cannot assume that caregivers can effectively identify and utilize family resources without developing skills in this area. Pratt et a1. (1987) feel that education, support groups, and service programs should provide opportunities for building skills in this area, and should reach beyond the primary caregiver to educate other family members about the needs of both caregivers and patients.

Support for family caregivers has become part of social and healthcare policy in several countries. Support services which are emphasized in the Scandinavian countries and the United Kingdom are day care, congregate housing, rehabilitation, respite, and preventive or health promotion services (USGPO, 1984). Although those services 
are also available in the United States, more liberal eligibility in the United Kingdom and Scandinavia makes them much more accessible in those countries (USGPO, 1984).

\section{SUMMARY}

There is ample documentation to indicate that being a family caregiver for impaired or elderly persons is often detrimental to the health and well-being of the caregiver. Analysis of numerous crosssectional studies indicate that the levels of burden, stress and other negative outcomes of caregiving such as depression do not necessarily diminish or disappear over time. In addition, studies have generally shown that there are characteristics of the caregiver him/herself and of the caregiving situation which may be more directly related to negative caregiver outcomes than the functional impairments of the care-recipient.

As indicated in the literature review, many caregiver studies are cross-sectional and include a heterogeneous group of caregivers in their samples. These limitations prevent an analysis of the impacts of caregiving over time and they also do not allow for an understanding of disease-specific or situation-specific associations with negative caregiver outcomes.

The study presented in this dissertation has a longitudinal design and the sample-selection criteria included only persons who were caregivers for community-dwelling persons experiencing a first stroke. Stroke patients who had other life-threatening or progressive illnesses prior to the stroke were not included in this study. The 
homogeneity of this sample as caregivers of a specific disease measured from onset, coupled with the longitudinal design, will contribute to an understanding of the specific issues and impacts of caring for persons disabled by stroke.

The following chapter describes the original study on which the fourth follow-up interview was based, and presents the major findings which have been reported from the original three waves of data collection. 


\section{CHAPTER II}

DESIGN, METHODOLOGY, AND RESULTS OF DATA FROM THE ORIGINAL STUDY

The study presented in this dissertation is a fourth wave of data collection with the primary support persons (caregivers) of stroke patients who participated in a panel survey entitled "A Longitudinal Study of the Psychosocial Impact of Stroke on Patients and Support Persons" (Schulz and Rau, 1984). The original study included three waves of data collection.

The following sections describe the sampling and data collection procedures for the original study, and the published results from analysis of caregiver data from $\mathrm{T} 1, \mathrm{~T} 2$, and $\mathrm{T} 3$. These sections are presented in order to provide the foundation for developing the conceptual framework, research questions, and hypotheses for the T4 study which is the focus of this dissertation.

\section{DESIGN OF THE ORIGINAL STUDY}

The study was designed as a longitudinal survey of persons who had recently-experienced a first stroke and their primary support persons. There were three waves of data collection: the first (T1) occurred from 3 to 10 weeks (Mean $=6.7$ weeks) after the stroke, the second (T2) occurred 6 months after the first interview, and the third (T3) occurred 6 months after T2. 
Structured in-person interviews of $11 / 2$ to 2 hours each were used to gather data from the primary support person and from the stroke patient if he/she could communicate well enough. The survey instrument was designed to include the collection of data about demographic and health variables, social support, stroke-related factors and psychological well-being. Most of the interviews were conducted in subjects' homes by several trained interviewers including the author.

\section{SAMPLING PROCEDURE}

One hundred sixty-two dyads of stroke patients and their caregivers (who were referred to as primary support persons throughout the original study) participated in the first wave of data collection. Referrals of potential subjects were made by staff at nine different hospitals in the Portland, Oregon and Pittsburgh, Pennsylvania areas. The primary support persons were identified by the stroke patients as persons who either had been or would be primarily responsible for their care after the stroke. Criteria for the inclusion of the primary support person in the study were:

1) the subject was identified by the stroke patient as being his or her primary support person,

2) the subject was available for in-person interviews and was willing to participate in the study,

3) the stroke patient was also willing to participate in the study. 
Since variables related to the stroke patient are included in the model of caregiver outcomes in this dissertation, it is also important to note the criteria for inclusion of the stroke patients in the study. These were:

1) medical evidence of a first completed stroke,

2) no evidence of co-existing terminal illness or other rapidly progressive medical condition,

3) occurrence of the stroke within three to ten weeks of the initial contact,

4) community dwelling status prior to onset of the stroke,

5) availability of a primary support person who was also willing to participate in the study,

6) willingness of the stroke patient to participate in the study if cognitively and verbaliy able to do so.

The sampling protocol for the first three waves of the study was to include both stroke patient and primary support person at each wave of data collection. A modified form of the interview was completed by the primary support person on behalf of the stroke patient if he/she was not cognitively or verbally able to participate. If the stroke patient died during the time of the study, the primary support person was interviewed (if willing) only at the next wave of data collection. Therefore, in cases where the stroke patient died between the first and second waves of data collection, caregiver data would have been collected only at $\mathrm{T} 2$ and not again at $\mathrm{T} 3$. 
Representativeness of the sample and generalizability of findings

The sample generated for $T 1$ is a non-probability sample. As mentioned above, careful attempts were made to define eligibility requirements so that findings over time could be attributed as much as possible to the effects of a documented first stroke, without being confounded by the presence of pre-existing life-threatening medical conditions or lack of clear definition of the nature of the stroke itself.

The findings from this study will have to be interpreted with caution because of the non-random selection of the sample. However, the strict adherence to eligibility requirements for participants enables us to be as confident as possible that the findings are reliably based on the experience of stroke and can therefore contribute to theory-building, future research, and policy development.

of the 162 stroke-patient/primary-support-person dyads who participated in the first wave of data collection, 140 participated in the second wave of interviews. One hundred twenty eight dyads completed all three interviews of the original study.

\section{CHARACTERISTICS OF THE ORIGINAL STUDY SAMPLE}

The following data are reported by Schulz, Tompkins, and Rau (1988) in their longitudinal analysis of primary support person data from T1 and T2, and by Tompkins, Schulz, and Rau (1988) in the analysis of data across the $T 1, T 2$, and $T 3$ waves of data collection. 
The primary support persons were mainly white $(86 \%)$ and female $(78 \%)$, ranging in age from 16 to 89 years (Mean $=56$ years). Eighty percent were either married or living as married; though not necessarily to the stroke patient, for an average of 27 years. Fiftyone percent were spouses of the stroke patients, $24 \%$ were children, and the remainder were primarily other relatives. Modal income for the support persons was between $\$ 30,000$ and $\$ 39,000$. Thirty-seven percent had graduated from high school and an additional 18\% had completed some college. Twenty-nine percent were working full time, $14 \%$ part-time, and $25 \%$ were retired at the time of the stroke.

The stroke patients themselves mere mostly white (86\%), male $(58 \%)$, and older than the primary support persons (Mean $=67$ years of age). The stroke patients' modal income at the time of the stroke was considerably lower than that of the primary support persons (between $\$ 5,000$ and $\$ 9,999)$. One-half of the stroke patients were retired at the time of the stroke, but almost one quarter (24\%) had been working full-time prior to the stroke.

Hospital discharge records received after the first interview documented that $52 \%$ of the stroke lesions had occurred in the left hemisphere, $36 \%$ in the right hemisphere, and $10 \%$ in the brainstem. Average functional disability for carrying out activities of daily living ( $A D L s$ ), as quantified by the Barthel Index (Mahoney and Barthel, 1965) was reported as being relatively mild at the first interview. On the Barthel Index the maximum score of 100 indicates functional independence. As measured at $T 1$, the average of the Barthel scores was 82 . Thirty-two percent of the stroke-patient 
sample was not cognitively or verbally able to respond to the questions and proxy interviews were conducted with the primary support person for this segment of the stroke-patient sample.

PREDICTORS OF DEPRESSION AT T1 AND T2

Schulz, Tompkins, and Rau (1988) report that being married to the stroke patient, concern about being able to care for the stroke patient in the future, and the objective severity of the stroke as measured by the Barthel Index were significant predictors of depression at T1. Neither demographic factors nor dimensions of social support significantly predicted depression at $\mathrm{T} 1$.

At T2 (seven to nine months post-stroke) the demographic variables of income, age, and health status were all significantly related to depression in the primary support person. Those subjects who were older, had better health and had higher incomes were less depressed than either younger subjects or those with lower incomes or poorer health. After controlling for those factors Schulz et al. (1988) report that the support person's direct rating of increases in the stroke patient's negative personality/behavior characteristics since the stroke was the primary variable associated with depression. Being married to the patient and concerns for future care of the stroke patient were not significant in predicting support person depression at $\mathrm{T2}$. 


\section{PREDICTORS OF CAREGIVER BURDEN AT T2}

A Caregiver Burden Scale, developed by Zarit (1980) and modified for use with caregivers of stroke patients by Rau (1986) was added at T2 and used in all subsequent interviews. Age was the only control variable associated with perceived burden at 12 , with older subjects feeling less burdened. Among the stroke-related variables, perceived severity of the stroke and concern for future care of the stroke patient (neither of which were significantly associated with T2 depression) were significant predictors of burden for the primary support person. In addition, caregivers who expressed less satisfaction with the amount of social contact they had with other people, and reported a greater decrease in the stroke patient's positive personality characteristics felt more burdened.

\section{CHANGE IN DEPRESSION SCORES BETWEEN T1 AND T2}

Schulz et al. (1988) report that although the mean scores on the depression scale did not change from $\mathrm{T} 1$ to $\mathrm{T} 2$, the scores of certain individuals changed considerably. Regression analysis of changes in depression with changes in predictor variables indicated that higher incomes were associated with decreasing depression (and vice versa) and that declines in reciprocal confiding relationships and. in satisfaction with amount of social contacts were significant in predicting increased depression. Similarly, increases in reciprocal confiding relationships and satisfaction with the amount of social contact were associated with decreased depression. 
PREDICTORS OF DEPRESSION AT T3

Tompkins, Schulz, and Rau (1988) report findings from a discriminant function analysis of $\mathrm{T} 3$ data. This analytical procedure was used in order to derive a means of classifying the support persons into optimal and less than optimal psychosocial outcome groups as defined by the depression scores. Based on the findings from T2 the significant demographic variables, stroke-related variables, social support variables, and T1 levels of depression and psychological wellbeing were entered into the analysis. The primary predictor of depression at $T 3$ was depression level at $T 1$. In addition, subjects most at risk for depression were more likely to be married to the patient than not, to name fewer people in their social networks, to have lower positive well-being scores at $\mathrm{Tl}$, and to be caring for younger stroke partners who had been more functionally impaired immediately after the stroke. Tompkins et al. (1988) suggest that al though the functional disability of the stroke patient was less at $T 3$ than at T1, the extent of personality and behavior change at $T 3$ was related to $\mathrm{T} 1$ initial functional disability for all subjects. The authors suggest that measurement of residual personality/behavior characteristics may be an important measurement of stroke severity over time. Lack of adequate recovery from this dimension of stroke has significant effects on the long-term adjustment and psychological well-being of the primary support person. 
SUMMARY

From analysis of the data gathered at specific intervals during the year following a documented first stroke, it is clear that primary support persons (caregivers) of the stroke patients are at risk for negative psychological effects which may impair their own long-term well-being. It was just this phenomenon that the author sensed while conducting T3 interviews with about a dozen of the caregiver subjects, and which led to the interest in doing a T4 wave of data collection. The following chapter describes the research questions of particular interest at T4, defines hypotheses to be tested which are derived both from the literature and the analyses of the original three waves of data, and presents the conceptual framework on which the analysis for this dissertation is based. 


\section{CHAPTER III}

\section{CONCEPTUAL FRAMEWORK FOR THE T4 STUDY}

\section{INTRODUCTION}

Although the occurrence of a stroke is a crisis event for both the person with stroke and the primary support person, the long-term adjustment for the caregiver may involve adjustment to a new role as "caregiver" as well as adjustment to the crisis event itself. As we have seen from the literature, the residual effects of stroke are physical, neurological, psychological, and social. Unless the stroke is very mild, most of the effects will be long-lasting, though not necessarily noticeable to anyone except those family members and friends who are in close, regular contact with the person who has had the stroke. The stroke creates a chronic health condition for the person who has had it; it also creates a new living condition for the caregiver of that person. The occurrence of a debilitating stroke creates a new situation which requires continued response and adjustment for both the patient and the caregiver. Since medical attention and physical rehabilitation for stroke patients is generally limited to a six month period (Kaufman and Becker, 1986), most longterm adjustment to stroke is accomplished by the stroke patients and 
the caregivers themselves with little professional guidance or support.

The purpose of the T4 study reported in this dissertation is to examine the psychosocial effects of providing care and support for a person with stroke beyond the first year after the stroke. Because the Tl data were gathered over a period of almost two years, the T4 data collection encompasses the period from two to almost four years post-stroke. In addition to providing the opportunity to assess the development of coping strategies and the experience of adjustment, the T4 study provides a unique opportunity to investigate long-term outcomes in relation to the initial levels of depressive symptomatology and psychological well-being.

The following sections present the model on which the analysis is based, the research questions of interest at $T 4$, and the hypotheses to be tested in this study.

THE MODEL FOR THE T4 STUDY

The model being tested in this study (Figure 1) is based on one developed by House $(1974,1981)$, George (1980), and Schulz, Tompkins, Wood and Decker (1987). Originally developed by House (1974) as a paradigm for stress research, it was adapted by George (1980) to show the process of transition to the roles of late life. Schulz et al. (1987) developed it as a support person coping model.

The objective occurrence of a loved one's stroke is the stressor in this model. Certain specific variables related to the stroke are 


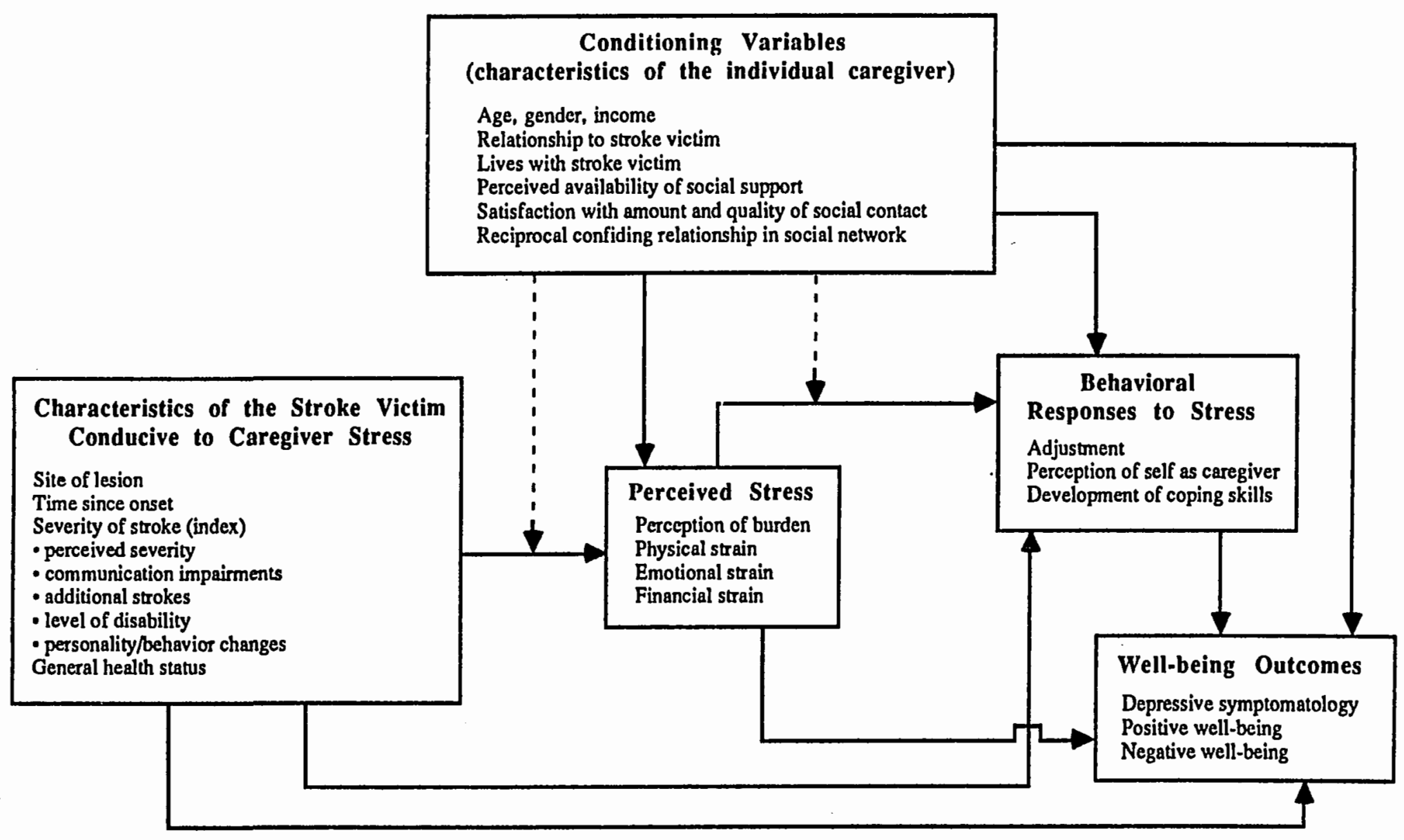

Figure 1. Support Person Coping Model after House (1974), George (1980), and Schulz et al. (1987). 
expected to have direct effects on stress, strain, and burden as experienced by the caregiver, as well as direct effects on the outcomes of depressive symptomatology, positive well-being, and negative well-being. These stroke-related variables include site of lesion (i.e. right cerebral hemisphere, left cerebral hemisphere, brain stem, or some other location such as the spinal cord), the amount of time since onset of the stroke, the severity of the stroke and level of disability of the person with stroke, the occurrence of additional strokes since $T 1$, and personality/behavior changes in the person with stroke. In addition to stroke-related variables, the general health status of the person with stroke will also be related to caregiver stress and well-being.

Certain characteristics of the caregiver are directly related to the caregiver's experience of stress and burden as well as to the outcome variables of depressive symptomatology, positive well-being and negative well-being. These characteristics include gender, age, income, relationship to the person with stroke (e.g. spouse, adult child, or other relative or friend), and the condition of living with the person with stroke versus living apart from him/her.

There is evidence that aspects of the caregiver's social support system will also have a direct effect on the experience of stress and burden and the levels of depressive symptomatology and positive or negative well-being. These include the perceived availability of support, as opposed to actual use of support (Kessler and McLeod, 1985; Ward, Sherman and LaGory, 1984); satisfaction with the amount of social contact and with the quality of social contact (Ward et al., 
1984; Williams, 1985; Schulz, Tompkins, Wood, and Decker, 1987), and the presence of at least one reciprocal confiding relationship in the caregiver's social network (Cohen and Wills, 1985). In addition to having direct effects on stress, burden, and psychological outcomes, these characteristics of the caregiver will also affect the coping processes used and the adjustment made to the stroke.

The social support variables which are included in characteristics of the caregiver are seen as potential mediators between the conditions of high stress and the outcomes of negative well-being. As such they serve a "buffering" role, and cause decline in negative outcomes in conditions of high stress. Other variables in this model which are seen to have buffering effects as well as main effects are the use of coping strategies and general adjustment to the stroke.

\section{RESEARCH QUESTIONS ADDRESSED BY THIS STUDY}

The fifteen research questions asked in this study address six specific areas of interest. The first area involves a comparison of the subjects who are still in a caregiving role with those for whom the person with stroke has died. What differences exist in the scores of the psychosocial outcome of depressive symptomatology, positive well-being and negative well-being between these two groups? Are respondents who are no longer in the caregiving role because of the death of the person with stroke better off than those who are still caregivers? 
The second area of interest relates to aspects of the stroke which affect the caregiver. What types of support and help do the subjects provide for the person with stroke? How much of this was specifically generated by the stroke? Are there specific long-term personality/behavior changes in the person with stroke which lead to high levels of perceived burden or result in high depressive symptoms over time? Is site of lesion a significant factor in caregiver outcomes? What aspects of the stroke are related to the caregiver's long-term adjustment to the stroke?

The third area of interest is in the correlates of long-term outcomes of depressive symptomatology, positive well-being and negative well-being. What variables at $T 4$ are significantly related to these psychosocial outcomes? What variables could be identified as predictors of depressive symptomatology at T4?

The fourth area of interest is in knowing if the primary support persons see themselves as "caregivers". There is much discussion in the field about whether or not persons who provide care for a disabled spouse, relative, or friend consider themselves to be "caregivers" or whether they interpret their involvement as reciprocal giving/receiving. Especially among married couples, the care "giver" may feel that he/she is simply living out the promises of the marriage vows and not doing anything special at all. Others consider that emphasis on the role of "caregiver" places the care recipient at a psychological disadvantage, and that such language can have a negative impact on the care recipient and perhaps also on the relationship. Since these observations are interesting and as yet anecdotal, a 
question asking respondents whether or not they do perceive themselves as caregivers was included in the study. The following research questions relate to this area: What percentage of the subjects perceive themselves as caregivers? Does this perception relate to adjustment to the stroke, to the experience of burden, or to the wellbeing outcomes?

The fifth area of interest is in examining the role which adjustment plays in the experience of strain and burden, and its relationship to the long-term psychosocial outcomes. Are there differences in the long-term outcomes between those respondents who feel that they have adjusted well and those who have not? What do respondents perceive as elements which contribute to adjustment to the stroke?

Finally, we want to know the effects of passage of time on caregiver outcomes and their experience of burden. Are there significant differences in the psychosocial outcomes or perception of burden between each point of data collection? Do negative personality/behavior changes in the person with stroke or specific elements of caregiver burden persist over time? Do some specific elements of stroke-related personality/behavior change or perceived caregiver burden improve (or decline) over time? Knowledge about these changes would be very helpful for understanding some of the components of long-term negative outcomes for the caregivers and provide useful information for the planning of caregiver interventions. 
HYPOTHESES TO BE TESTED

Based on the literature presented in Chapter $I$ and on the analyses of the sample at T1, T2, and T3 which were presented in Chapter II, several hypotheses regarding the long-term outcomes of caregiving for a person with stroke will be tested in the analyses of T4 data.

I. It is predicted that caregivers who live with the person with stroke will exhibit higher depressive symptomatology, higher negative well-being, and lower positive well-being than those who live in different households.

II. It is predicted that the long-term severity of the stroke, measured by an index which includes the amount of assistance needed by the stroke patient, the negative personality/behavior changes which have occurred as a result of the stroke, and the stroke patient's ability to communicate with the caregiver, will be significantly related to well-being outcomes. Specifically, moderate to high stroke severity at T4 will result in high perceived stress, high depressive symptomatology and negative well-being, and low positive well-being.

III. It is predicted that perceived stress, which includes measures of strain and burden, will be positively related to depressive symptomatology and negative well-being, and negatively related to positive well-being.

IV. It is predicted that two sets of independent variables will have buffering effects between perceived stress and the outcome measures. These are a) social support and b) responses to stress. 
A. Under conditions of high perceived stress, caregivers who are satisfied with the amount and quality of their social contacts, who have at least one reciprocal confiding relationship in their social network, or who perceive that they have instrumental assistance from their social support system available if they need it will be less depressed and exhibit higher positive well-being than those who do not.

B. Under conditions of high stress, caregivers who have developed coping strategies to deal with the stroke will have significantly lower depressive sympiomatology and negative wellbeing, and higher positive well-being than caregivers who do not.

The methodology used to gather data at T4 and to perform the analyses in this study are presented in Chapter IV. 


\section{CHAPTER IV}

\section{METHODOLOGY}

The study presented in this dissertation is a Time 4 (T4) follow-up study conducted with the primary support persons (caregivers) of stroke patients who participated in a panel survey entitled "A Longitudinal Study of the Psychosocial Impact of Stroke on Patients and Support Persons (Schulz and Rau, 1984). A description of the original study and the major findings from its three waves of data collection are presented in Chapter II.

The following sections describe the sampling and data collection procedures, the operational definitions of the variables measured, and the procedures used for data analysis at T4.

\section{THE SAMPLE}

The sample for the study described in this dissertation was drawn from the caregivers who participated in the Schulz and Rau (1984) study described above. A full description of the sampling procedure used at $\mathrm{T} 1$ and a demographic description of the $\mathrm{T} 1$ sample of caregivers is presented in Chapter II.

Sampling procedure at T4

The interest at T4 was in looking at the long-term aspects of caregiving, and also in noting comparisons between respondents who were no longer caring for the stroke patient and those who were. In 
order to investigate this comparison, respondents for T4 were drawn from the sample of all respondents in $T 1 \quad(n=162)$ rather than just from those who had completed all three earlier interviews ( $n=128)$.

A letter was sent to all respondents who had participated at T3 plus those who had dropped from the study because of the death of the stroke patient (Appendix A). The total number of letters sent to potential respondents was 132 . The letter described the intent of the follow-up study and mentioned that the interviewer would telephone the caregiver to answer any questions and to set an appointment for a subsequent telephone interview if the caregiver was willing to participate.

At Time 1 a letter of consent, approved by the Human Subjects Research Review Committee, had been signed by each of the respondents (Appendix B). An additional letter of consent was not requested at Time 4 since the interview was conducted over the telephone and could easily be refused or terminated by the caregiver at will.

One hundred and fifteen persons were able to be located from the T1 sample. Of these, ninety-three participated in the T4 interview. This figure represents fifty-seven percent of the original sample and eighty-one percent of the respondents available at T4. Table I summarizes the reasons for non-participation at T4.

\section{DATA COLLECTION}

The first three waves of data collection were carried out between October 1983 and August 1986. The initial wave (T1) occurred from three to ten weeks after the documented first stroke (Mean $=6.7$ 
TABLE I

REASONS FOR ATTRITION BETWEEN TIME 1 AND TIME 4

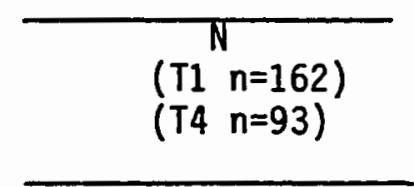

Refused further participation at T2 or T3 30

Not locatable 20

Caregiver's health too poor to participate 3 or to complete the interview

Person with stroke too $i 11$, caregiver occupied 1 with care recipient

Recent death of person with stroke 3

Not available for interview during period of
data collection

Caregiver died between T3 and T4 3

Refused because care-recipient had
died early in the study

Refused: "no time", "person with stroke is doing fine" $\underline{3}$

Total \# non-participants in T4 69 ( $43 \%$ of $\mathrm{Tl}$ sample)

weeks ). The second wave (T2) occurred six months later, and the third (T3) occurred six months after Time 2.

The fourth wave of data collection (T4) was carried out between June 1987 and January 1988, occurring between 24 and 42 months after the first wave (Mean $=36.5$ months, S.D $=7.5$ ). The T4 interview was conducted by telephone, a method used for other followup studies of caregivers (Morycz, 1985). 
The primary data collection tool for the first three waves was a structured in-person interview. This interview included several selfreport measures which were completed by the caregiver either at the time of the interview or were left with the caregiver to complete and mail to the interviewer. The primary data collection tool for the fourth wave was a structured telephone interview which was designed by the author (Appendix C). The self-report measures which had been administered in the first three waves were also used at T4. They were sent in the mail after the telephone interview was completed and were returned by mail to the interviewer.

The in-person interviews for the first three waves were carried out by four experienced interviewers, including the author. The author also conducted eighty percent of the telephone interviews for the T4 study. The remainder of the T4 interviews were conducted by a researcher who had worked on the earlier study. Pre-tests of all interviews were carried out and close contact was kept between the interviewers to assure consistency at each wave of data collection. The data reported in this dissertation are from respondents who either participated in all four waves of data collection, or who participated through the death of the person with stroke and again at T4.

\section{VARIABLES - OPERATIONAL DEFINITIONS}

The data for the variables to be measured were gathered from four general sources. Two sources were the interview questions designed specifically for the first three waves of data collection and 
the questions specifically designed for the fourth wave (Appendix C). All four interviews also utilized standardized instruments that had been frequently used and tested. Hospital discharge records provided data on the type of stroke and site of lesion.

The variables in this study were divided into five general categories: characteristics of the caregiver, characteristics of the stroke and stroke patient which would potentially affect caregiver well-being, perceived stress, behavioral responses to stress, and outcome variables of caregiver well-being. Figure 2 shows the measurement of variables at each time period.

\section{Characteristics of the caregiver}

Age. Respondents gave their age at the time of each interview. Gender. Gender of the caregiver was observed by the interviewer at the time of the first interview.

Income. Income was defined as total household income prior to the stroke. At the first interview respondents were asked to indicate their income category on a scale fron (1) less than $\$ 5,000$ to (9) more than $\$ 50,000$. Because this variable was originally measured in uneven categories (increments of $\$ 4,999$ up to $\$ 29,999$ and then increments of $\$ 9,999$ up to $>\$ 50,000$ ) it was recoded to midpoint for correlational and t-test analyses. Income at $\mathrm{Tl}$ was used for analysis with outcomes at T4 and also in a t-test to determine differences between respondents who had dropped from the study after $\mathrm{Tl}$ and those who had participated at T4. A direct measure of change in standard of living 


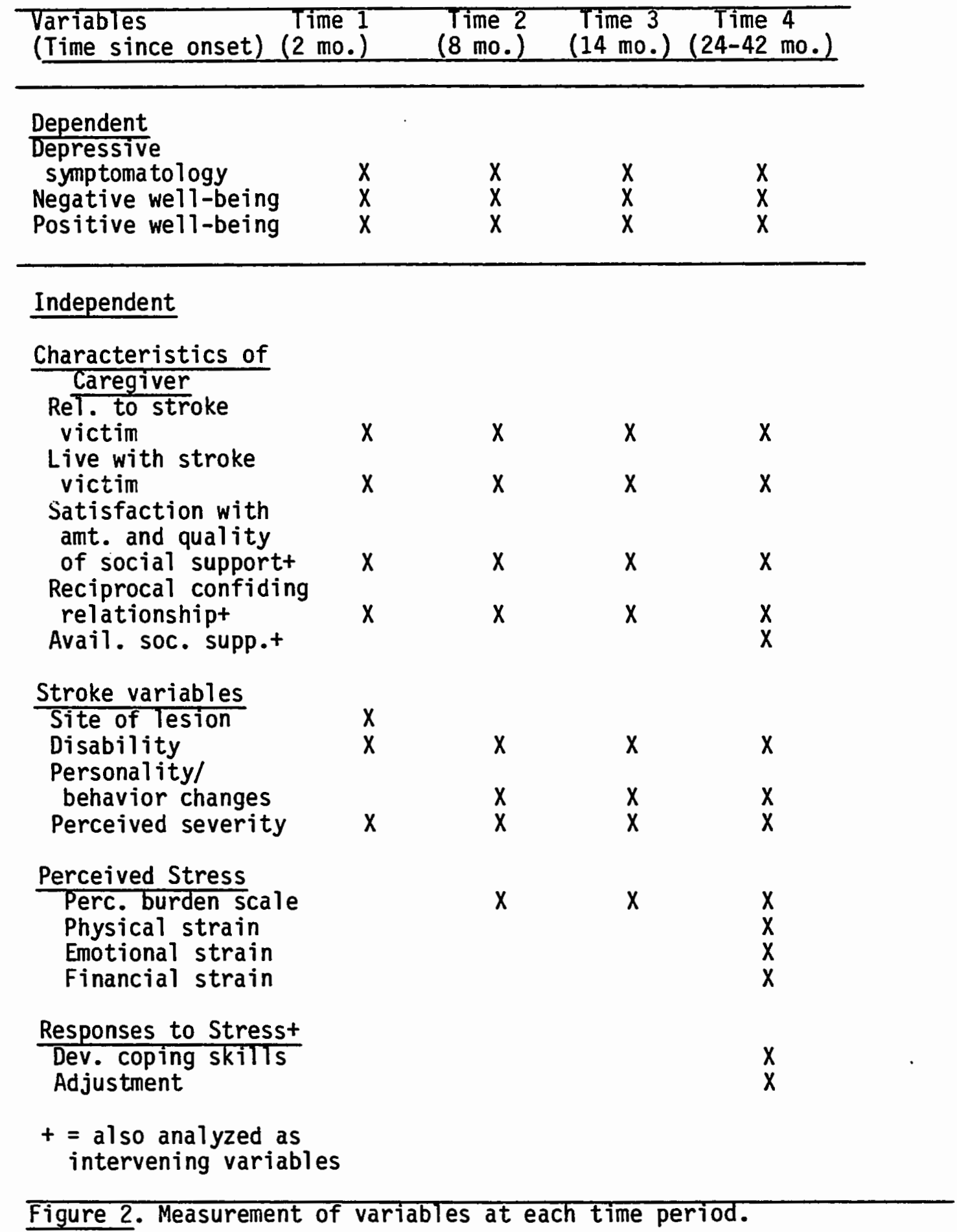


(economically) was used at T2, T3, and T4. Respondents were asked if their standard of living had changed since the previous interview; if it had changed they were asked if it were now better (1) or worse (0). Subjective health status. Respondents were asked to rate their overall health at the present time as excellent (1) very good (2) good (3) fair (4) or poor (5). In addition, they were asked if their health is now better (1) the same (2) or worse (3) since the last interview. Both variables were later recoded in the opposite direction so that 'excellent' and 'better' were at the high end of the scale and 'poor' and 'worse' were at the low end.

Objective health status. Respondents were asked to name any chronic illnesses which they have at $T 4$, and to indicate which of those (if any) had come about since the stroke.

Relationship to stroke patient. Respondents indicated their relationship to the stroke patient at the first interview. Categories of relationship were (1) spouse (2) child (3) sibling (4) parent (5) in-law (6) niece/nephew (7) grandparent (8) aunt/uncle (9) grandchild (10) neighbor (11) work colleague (12) friend (13) spousal friend (14) other. This variable was recoded in the analysis at T4 to (1) spouse, spousal friend (2) child (3) other, and also to (1) spouse and (2) non-spouse for comparative analyses with reported results from T2 and T3 analyses (Schu1z et a1., 1988; Tompkins et a1., 1988).

Lives with stroke patient. Respondents were asked where the stroke patient was living. Categories included (1) alone in own home, apartment, or mobile home (2) with caregiver (3) with other family member or companion (4) foster home or licensed care facility (5) 
hospital (6) other. After initial analyses this variable was recoded into two categories: (0) stroke patient does not live with the caregiver and (1) stroke patient lives with the caregiver.

Presence of reciprocal confiding relationship in social network. As in the earlier interviews, respondents were asked to name persons with whom they had talked about their own personal matters during the previous year. That question was followed by one in which they were asked to name persons who had talked with them about personal matters in the previous year. Names which appeared as answers to both questions were considered to be a "reciprocal confiding relationship". Correlational analyses were conducted with interval data (number of persons named as being reciprocal confidants) and also with dichotomous categories of presence (1) or absence (0) of reciprocal confidants.

Perceived availability of instrumental social support. Following the questions in which respondents named the persons with whom they had confiding relationships, respondents were asked, "Are there people you would ask to assist you with transportation, chores, cooking, or who would provide occasional time off for you if you needed such help?" Respondents then gave the first name and relationship of each of these persons. The names were summed to indicate amount of perceived availability of instrumental support.

Satisfaction with amount of social contact. Respondents were asked to indicate their satisfaction with the amount of social contact they have at the time of the interview on a five point scale ranging from (1) very dissatisfied to (5) very satisfied. 
Satisfaction with the quality of social contact. Respondents were asked to indicate their satisfaction with the quality of social contact they have at the time of the interview on a five point scale ranging from (1) very dissatisfied to (5) very satisfied.

\section{Characteristics of the Stroke}

Site of lesion. The site of lesion refers to area of the brain or central nervous system affected by the stroke. Site of lesion classification in this study indicates that the stroke has occurred either in the left cerebral hemisphere, the right cerebral hemisphere, the brain stem, or elsewhere (e.g., the spinal cord). Data for this variable were taken from the hospital discharge records received at the time of the first interview.

Time since onset. This variable was measured as number of months since the $\mathrm{T} 1$ interview, which occurred within 2 months (Mean = 6.7 weeks) of the specific onset of the stroke.

Severity of stroke. Several different measures were used to to measure severity of stroke. These were (1) the Barthel Index, a widely used index of functional independence in the activities of daily living (Mahoney and Barthel, 1965); (2) the perception of the extent of the care-recipient's physical problems; (3) the ability of the care-recipient to communicate with the caregiver and with other people; (4) the occurrence of additional strokes and (5) the extent of personality and behavior changes since the stroke.

The Barthel Index assigns values of $0,5,10$, or 15 to specific capabilities on each of ten activities of daily living (ADLs). 
Examples of ADLs would be eating, dressing, toileting, mobility, etc. A maximum score of 100 is obtained when the person is completely independent. Respondents were asked to rate the person with stroke on the Barthel Index at all four times of data collection. The internal consistency of the T4 sample on the Barthel Index was high $(a=.91)$.

The respondents' perception of the extent of the carerecipient's physical problems and need for assistance was measured on a five-point scale ranging from (1) no problem and (2) mild problem, needs little or no help to (5) very severe problem, needs help with almost everything.

The ability of the person with stroke to communicate with the caregiver, as well as his/her ability to communicate with others, were both measured on a four-point scale, ranging from (1) speaks and is usually understood to (4) no communication.

Additional strokes were measured by asking if the care-recipient had experienced more medically diagnosed strokes since the first one.

Personality and behavior changes since the stroke were also considered to be a measure of stroke severity. These were measured by a scale of 19 items (6 positive and 13 negative) generally considered to be associated with stroke (Rau, 1986). Respondents were asked if the stroke patient exhibited these characteristics more, less, or about the same as before the stroke. Scores were calculated as total number of increased negative behaviors, total number of increased positive behaviors, total number of decreased negative Dehaviors, total number of decreased positive behaviors, and total number of behaviors which remained about the same. 
An index of stroke severity to complement the Barthel Index of functional disability was formed by summing the z-scores of the the following items: perceptions of the care-recipients physical problems, ability of the care-recipient to communicate with the caregiver, and increased negative behaviors (which included both negative behaviors which had increased and positive ones which had decreased). The standardized alpha of internal consistency for this index was adequate $(a=.66)$.

General health status of the person with stroke. Respondents were asked to to indicate if the care recipient had any chronic illnesses other than the effects of the stroke, and also if the care recipient had been hospitalized in the past year (and why). Respondents were also asked to indicate if the health of the person with stroke was better (1), the same, (2) or worse (3) since the last interview. Health was subsequently recoded in the opposite direction (better $=3$, worse=1) in order to clarify the direction of relationships in correlational analyses.

Caregiver tasks related to the stroke. Respondents were asked to respond to a list of different types of assistance which people who have had strokes may need. They were asked to indicate if the person with stroke is able to do that task for him/herself, if the respondent does the task, and if family, friends, private pay assistant, or an agency helps with that task. In addition, respondents were asked to indicate the frequency with which each provider did each task. The list of tasks was: meal preparation, feeding, dressing, bathing, walking/help with mobility, transportation, shopping, household 
chores, house and yard maintenance, general supervision, visiting or keeping the person with stroke company, paying bills and doing correspondence, phone check-up, someone to stay with the person with stroke when respondent is gone, and 'other' (specify). Frequencies were: same as before stroke (0), several times a year (1) monthly (2) 2-3 times a month (3) weekly (4) 2-4 times a week (5) 5-6 times a week (6) and daily (7).

\section{Perceived Stress}

Respondents were asked to indicate how much physical, emotional, and financial strain they have experienced overall in caring for the person who had a stroke. Each of these items was measured on a five-point scale (1) not at all to (5) quite a lot.

Perceived burden was measured by Rau's (1986) adaptation of the Caregiver Burden Scale developed by Zarit et al. (1980). It is a 14item Likert-type scale which measures the perception that providing care for the person with stroke is interfering with such things as personal time, relationships with family and friends, social life, health, and other responsibilities. In addition, it taps dimensions of burden such as resentment that other family members are not as involved as they might be, feelings that the person with stroke is demanding more of the caregiver than is necessary, or feelings that the person with stroke does not appreciate what the caregiver is doing on his or her behalf. Each of the items is measured on a five-point scale of never (0) to almost always (4). Included in the scale are two items of positive experience in caregiving: feeling that one is 
contributing to the stroke patient's well-being, and feeling usefur in personal interactions with the stroke patient. These two items are coded in reverse order (never $=4$; always $=0$ ). The internal consistency for this scale at T4 was high $(a=.88)$.

\section{Responses to Stress}

Development of Coping Skills. A modified version of the F-Copes scale (McCubbin, Larsen, and 01son, 1981) was used to measure coping skills, with each item measured on a three-point interval scale. Fifteen phrases describing various coping skills were given and respondents were asked to indicate if they used these $(0)$ not at all (1) sometimes or (2) often. The 15 coping skills tapped several dimensions: (a) use of the informal support system (friends, relatives) for support and encouragement, (b) use of community resources (church, community agencies, support group) for support, or assistance, or advice, (c) use of health care professionals (physician, caregiver training courses) for advice or training, and (d) self-care (taking time to do things for self, having faith in God, using relaxation techniques or exercise). The questions were introduced in the following manner:

"People respond to new roles in many different ways. I'd like you to indicate how much the following statements describe the things you have done to adjust to the role of supporting (name of stroke patient) after the stroke. As I read the items, think about yourself and tell me how often you cope in these ways. The choices are sometimes, often, or not at all. 
" When I have faced problems or difficulties I have responded by (1) sharing my difficulties with relatives, (2) seeking support and encouragement from friends, (3) attending church services, (4) seeking information and advice from persons in other families who have faced the same or similar problems, (5) seeking advice from relatives (6) participating in church activities (7) seeking assistance from community agencies and programs designed to help families in our situation, (8) sharing concerns with close friends, (9) seeking advice from a minister, (10) making sure I have some time to do things just for myself (11) joining a caregiver support group, (12) having faith in God, (13) taking a caregiver training course, (14) seeking advice from our physician, (15) using relaxation techniques or exercise." The alpha level of reliability of the coping strategies scale for this sample was moderate $(a=.72)$.

In addition, respondents were asked, "Are there other ways in which you have coped that were not on the list?" Verbatim responses were recorded.

Perception of self as caregiver. Respondents were asked to indicate (yes/no) if they considered the care recipient as dependent on the caregiver "for care or support because of the stroke". Respondents were then asked "Do you think of yourself as a caregiver for __?". Responses were coded into three categories: (0) no (1) sometimes (2) yes. In addition to analysis in these three categories, this variable was recoded and analyzed as a dichotomous variable (no, yes). 
Adjustment to stroke. Respondents were asked how well they felt they had adjusted to the stroke. Categories were (0) not at all (1) somewhat (2) pretty well (3) very well.

Perceived adjustment of person with stroke to the stroke. Respondents were also asked how well they felt the care-recipient had adjusted to the stroke. Categories were (0) not at all (1) somewhat (2) pretty well and (3) very well.

Outcome variables

Two standardized instruments were used as outcome measures in this study: the Center for Epidemiological Studies Depression Scale (CES-D; Radloff, 1977) and the Index of Psychological Well-Being (IPWB; Berkman, 1971).

Depressive Symptomatology. The Center for Epidemiological Studies Depression Scale (CES-D; Radloff, 1977) was constructed from other validated depression scales and was specifically designed for use with community samples. It has been used in many studies and has norms for various age groups. It has been cited as particularly useful with older persons because it was designed to avoid placing too much emphasis on somatic symptoms which are frequently experienced by nondepressed older persons (Williams, 1985). Although it is not a measure used to diagnose clinical depression as defined by the Diagnostic and Statistical Manual of Mental Disorders (DSM-III, American Psychiatric Association, 1980), Price (1987) notes that in a study validating the use of the CES-D with stroke patients all of the respondents who had scores of 16 and above (the cutoff for indicating 
risk for clinical depression) on the CES-D had been diagnosed as clinically depressed. Radloff (1977) reports a coefficient of internal consistency of .85 for the CES-D. The Cronbach's alpha for internal consistency of the CES-D for this sample at T4 was high ( $a=$ $.90)$.

Index of Psychological Well-Being (IPWB). This index is an eight item self-report scale adapted by Berkman (1971) from the Bradburn Affect Balance Scale (Bradburn, 1969) to measure mental health in a general population. It consists of positive and negative feelingstate items, and is designed to indicate the relative strength of the caregiver's positive and negative feelings. Separate scores are computed for positive well-being and negative well-being, and they may also be combined on a matrix to describe a balance score of psychological well-being.

Because positive and negative well-being have been found to be independent constructs, especially when frequency rather than intensity is measured (Diener, 1984) positive and negative well-being have been analyzed separately in this study. Internal consistency of the positive well-being scale at T4 was moderately high $(a=.78)$, as was the reliability of the negative well-being scale $(a=.80)$.

\section{DATA ANALYSIS PROCEDURES}

The statistical analyses described in this study were done using SPSSX, Second Edition (1986) and SPSSPC+ (Norusis, 1986). Analyses of qualitative data were done using content analysis. 
Descriptive Analys is

General descriptive statistics including frequency, measures of central tendency, and standard deviation were computed for all variables at T4. Descriptive statistics were also computed for the respondents in this study on selected variables at $\mathrm{T} 1, \mathrm{~T} 2$, and $\mathrm{T} 3$. The variables chosen from the first three waves of data collection included demographic variables, stroke-related variables, predictor variables, and outcome variables. A list of variables and the points in time at which they were measured was presented in Figure 2.

\section{Comparison of respondents who participated at $\mathrm{T} 1$ with those who}

participated at $T 4$

Before examining relationships between variables at Time 4 and also across the other points of data collection, t-tests were conducted to determine if there are significant differences between those respondents who participated in Time 1 and subsequently dropped out of the study, and those who remained in the study. T-tests on demographic variables and outcome variables showed significant differences on income and years of education between participants and non-participants at T4. Table II illustrates the means, standard deviations, and t-test results of demographic and outcome variables between the $\mathrm{T} 1$ and $\mathrm{T} 4$ participants.

Analysis of relationships between variables at T4 and demographic variables from $\mathrm{T} 1$

Since demographic variables (gender, relationship to person with stroke, residential setting and socio-economic status) and 
medical documentation of certain stroke variables were collected only at $\mathrm{Tl}$, it was necessary to include these specific $\mathrm{Tl}$ variables in an examination of the relationship of T4 variables to the outcome measures of psychological well-being at T4.

Statistical procedures used to analyze relationships at T4 included Pearson product-moment correlation, chi-square, and analys is

\section{TABLE II}

MEANS, STANDARD DEVIATIONS AND T-TEST RESULTS FOR SUBJECTS PARTICIPATING IN TIMEI VERSUS PARTICIPANTS IN BOTH TI AND T4

\begin{tabular}{|c|c|c|c|c|c|}
\hline \multirow[b]{2}{*}{ VARIABLE } & \multicolumn{2}{|c|}{$T 1, \cot _{(N=69)}^{T 4}$} & \multicolumn{2}{|c|}{$\begin{array}{c}\text { T1 AND T4 } \\
(N=93)\end{array}$} & \multirow[b]{2}{*}{ t-value } \\
\hline & Mean & S.D. & Mean & S.D. & \\
\hline AGE & 55.3 & 17.1 & 56.5 & 13.1 & -.48 \\
\hline INCOME & $19,968.00$ & 15,369 & 27,577 & 17,567 & $-2.84 * \star$ \\
\hline YEARS OF EDUCATION & 12.5 & 2.6 & 13.2 & 2.2 & $-1.99 \star$ \\
\hline $\begin{array}{l}\text { BARTHEL INDEX OF } \\
\text { STROKE SEVERITY }\end{array}$ & 83.7 & 19.0 & 81.3 & 18.7 & .76 \\
\hline CES-D (20-item) & 13.4 & 9.1 & 12.0 & 8.7 & .95 \\
\hline POSITIVE WELL-BEING & 4.4 & 1.9 & 4.1 & 1.8 & .91 \\
\hline NEGATIVE WELL-BEING & 4.3 & 2.7 & 3.6 & 2.4 & 1.70 \\
\hline
\end{tabular}

of variance. Reliability analyses of all scales and indices were carried out using Cronbach's alpha test of internal consistency.

Multiple linear regression procedures were used to test the relative importance of predictor variables in relation to the outcome 
measures of depressive symptomatology, caregiver burden, and psychological well-being.

Analysis of Change Over Time

Change over time was analyzed in several ways. Analyses of change over each of the four points of data collection in the outcome variables (depressive symptomatology, negative well-being, and positive well-being) and in the intervening variable of perceived burden were conducted by repeated measures analyses of variance. In addition, a descriptive analysis approach was used to present comparisons of the proportion of the sample who reported high levels of depressive symptomatology, high levels of specific items of caregiver burden, and high levels of negative changes in the personality/behavior characteristics of the stroke patient at each point of measurement. Changes over time in the stroke patients' ability to transport him/herself, and in the use of stroke clubs or caregiver support groups by the caregivers were also analyzed descriptively.

\section{Presentation of Findings}

The following chapters present the results of the data analysis described above. Chapter $V$ reports the demographic characteristics of the sample and describes the nature of the caregiving role as experienced by this sample. Chapter VI discusses the relationships of variables as measured at $T 4$, including the results of multiple regression analyses, and Chapter VII presents findings related to changes over time. 


\section{CHAPTER $V$}

\section{DESCRIPTIVE ANALYSIS}

\section{INTRODUCTION}

One of the interests in this study was in gathering information from ali of primary support persons about their caregiving experiences since the stroke, and to examine potential differences between those who were no longer in the caregiving role because of the death of the person with stroke and those who were still in the role of being a primary support person to the stroke patient. The descriptive analyses in this chapter report data from all of the caregivers at T4 $(n=93)$. However, the section on caregiver tasks and the remaining analytical chapters which investigate the relationships between variables as they effect long-term well-being outcomes for the caregivers will report data only on the caregivers who were still in the role of primary support person at T4 $(n=81)$. All comparisons across time in this dissertation are for the caregivers in the T4 sample on1y.

The general characteristics of the sample which are presented in this chapter include demographic data and information about the caregiver's social support system as well as demographic data about the persons with stroke and the characteristics of the stroke which are conducive to caregiver stress. Descriptive data are also included 
for variables which measure caregiver stress, responses to stress, and the outcomes of depressive symptomatology and psychological wellbeing. In addition, the types of tasks which the caregivers are still providing for the person with stroke at T4 and the types of assistance which they receive from the formal and informal support systems are presented.

The caregivers in this study were specifically named by the stroke patients at the time of the stroke as being the primary person who would be responsible for providing care and support for them following the stroke.

\section{DEMOGRAPHIC CHARACTERISTICS OF THE CAREGIVERS}

Demographic data for the sample are summarized in Table III. The pre-stroke median household income for the sample $(\$ 22,500)$ was higher than that reported for the U.S. population as a whole $(\$ 16,830$; Bureau of Census, 1980). The educational level of this sample was also higher. Fifty percent of the sample had more than 12 years of formal education compared with the national average of $32 \%$. As reported earlier, caregivers with lower income and fewer years of education were more likely to drop out of the study by T4.

The median age of the caregivers in the T4 sample is 60 years. Ethnic representation is predominately Caucasian. The following sections discuss in detail the specific demographic characteristics of the sample. 
TABLE III

GENERAL DEMOGRAPHIC DATA FOR ALL CAREGIVERS

AGE $(\mathrm{N}=93)$

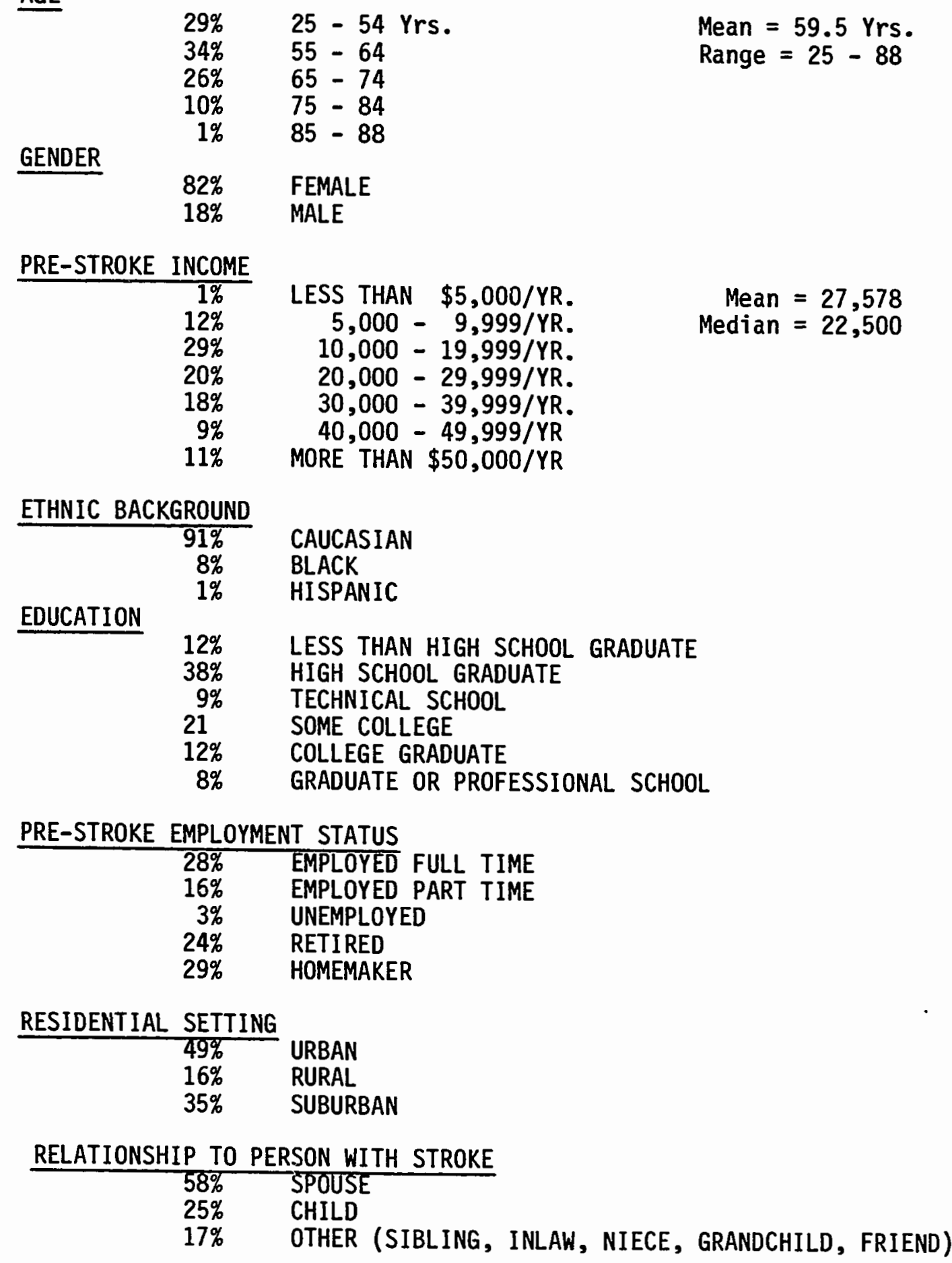


Age

The ninety-three caregivers in the sample had a mean age of 59.5 years, S.D. $=12.87$, with a range of 25 to 88 years at T4. Table III shows the age categories in detail. Those who were still caregiving at T4 $(n=81)$ were providing care and support to care-recipients with a mean age of 70.4, S. D. $=11.03$, ranging from 40 to 92 years of age. These figures are similar to large random-sample caregiver studies. In the 1980 National Long-Term Care Demonstration (known as the Channeling Demonstration; Stephens and Christianson, 1986) $40 \%$ of the nearly 2,000 caregivers were over 65 and 15\% were over 75 . Data from the Informal Caregivers Survey (a subset of the 1982 National LongTerm Care Survey, with over two million caregivers aged 14 years and older) indicate that the average age of the caregiver population is 57 years. Estimates from the Informal Caregivers Survey indicate that twenty-five percent of all caregivers are between 65 and 74 years of age, and another 10\% are 75 years old or older (Stone, 1987). The age categories in this sample are comparable to those in both of the national caregiver studies.

\section{Gender}

Eighty-two percent of the caregivers in this sample are female. This figure is somewhat higher than national estimates from the Informal Caregivers Survey (Stone, 1987), which indicate that approximately 72 percent of caregivers are female, and the National Long-Term Care Demonstration (Stephens and Christianson, 1986) in which $73 \%$ of the caregivers are female. The higher ratio of females in 
this sample may be related to the inclusion of only stroke caregivers in the study, reflecting the statistic that men are more at risk for stroke than women (Dyken, 1987) and therefore there may be a higher ratio of female spousal caregivers in a stroke study than in other types of caregiver studies.

Relationship to the person with stroke

Fifty-six percent of the caregivers were married to the persons who had experienced the stroke, and two percent were living as married. Twenty-five percent were children of the care-recipient, and $17 \%$ were other relatives or friends.

\section{Marital Status}

Eighty-one percent of the caregivers wers married, and two percent were living as married, at the beginning of the study (T1). Of the non-spousal caregivers $(n=39)$ 64\% were married, $8 \%$ were widowed and $19 \%$ were separated or divorced. At $14,11 \%$ of the sample indicated a change in marital status since the year following the stroke (T3). All of the marital changes reported at T4 occurred among non-spousal caregivers. In national data, seventy percent of all caregivers are married, and about half of both male and female nonspousal caregivers are married.

\section{Education}

The caregivers in this study tend to be more educated than those in the National Long-term Care Demonstration project (1980), with $30 \%$ having completed college compared to $15 \%$ in the national sample. Only 
one percent of the sample described in this study had stopped formal education at the junior high level, and none of the caregivers had less than junior high school education. This compares with $24 \%$ of the national sample reporting either no formal education or only elementary school education. Educational level was not reported for the Informal Caregivers Survey. Unfortunately, caregivers with fewer years of education were lost to the study by Time 4 ( $t$-value between T1 and T4 caregivers was $-1.99 \mathrm{p}<.05)$.

Income

The mean income of this sample at $T 1$ was $\$ 27,578$, S.D. $\$ 17,567$, and the median income was $\$ 22,500$. The majority of caregivers in the National Long-Term Care Survey reported adjusted family incomes in 1982 which fell in the middle income bracket, but a little less than one-third of that population had incomes in the poor or near poor category (Stone, 1987). The sample discussed in this dissertation also had incomes in the middle bracket, with about $13 \%$ indicating poor or near poor levels of income.

T-tests comparing the income level of caregivers who dropped out of the study indicated a significant difference in income between those caregivers who remained in the study for T4 and those who did not participate at T4. Those who did not participate at T4 had. an average income of $\$ 19,968$, S.D. $\$ 15,369(t=-2.84, p>.01)$.

At T4, 18\% of the caregivers indicated that their standard of living had declined since the the year following the stroke (T3), but 
$10 \%$ indicated that their standard of living had improved since that time.

Employment Status

At the beginning of the study (T1) about one-third (28\%) of the caregivers in the T4 study were employed full time, $16 \%$ were employed part-time, $24 \%$ were retired, $29 \%$ were largely homemakers, and $3 \%$ were unemployed. Sixteen percent reported a change in employment since the year following the stroke (T3) and $8 \%$ of those indicated that the employment change was related to the stroke. Although some of those caregivers had stopped working in order to provide more care to the person with stroke, at least one had gone back to work in order to replace income which was lost because of disabilities caused by the stroke.

\section{Residential setting}

At the time of the stroke, forty-nine percent of the caregivers lived in an urban setting, $35 \%$ in a suburban setting, and $16 \%$ in a rural area.

Eighty-five percent owned their own homes. Ten percent of the caregivers had moved since $T 3$, but only $2 \%$ of those indicated that the move was related to the stroke. One couple in that category had moved in order to be closer to an adult child.

Health Status

At $\mathrm{T} 118 \%$ of the caregivers in the $\mathrm{T} 4$ sample reported their health as excellent, $27 \%$ as very good, $41 \%$ as good, $10 \%$ as fair, and 
$4 \%$ as poor. At T4 $20 \%$ of the sample reported their health as excellent, $26 \%$ as very good, $32 \%$ as good, $15 \%$ as fair, and $7 \%$ as poor. In a direct measure of change in health since the last interview, $21 \%$ of the caregivers reported that their health had declined. However, fifteen percent reported improved heal th since T3.

Sixty-one percent of the caregivers reported having at least one chronic illness at T4. Two percent of the sample developed their first chronic illness after the onset of the care-recipient's stroke, and $25 \%$ developed at least one additional chronic 111 ness after the stroke. Twelve percent of the caregivers had been hospitalized in the past year.

Of the caregivers in the National Long-Term Care Demonstration, $60 \%$ reported that their own general health was excellent or good, and almost $13 \%$ stated that they were in poor health (Stephens and Christianson, 1986). One fourth of the caregivers in the National Caregivers Survey reported that they were in excellent health, and about one-third of that sample perceived its health status as fair or poor.

Given these comparisons, and the variation in measuring specific categories of health status, it appears that the caregivers discussed in this dissertation are similar in health status to those in the national samples.

\section{Living Arrangement}

At T4 56\% of the caregivers were living with the person who had experienced the stroke. Fifty-two percent of these were spouses or 
spousal friends and the other $4 \%$ were children or other relatives. This is somewhat less than reported in the National Long-Term Care Demonstration, in which $66 \%$ of the caregivers lived with the care recipient. It is considerably lower than the national estimate that almost $75 \%$ of caregivers live with the functionally disabled family member (Stone, 1987). Twelve percent of the persons with stroke were living in a long-term care facility at the time of the T4 interview.

\section{CAREGIVER TASKS, ASSISTANCE, AND RESPITE}

Table IV illustrates the types and frequencies of tasks which the caregivers were providing for the person at T4, several years after the onset of stroke. Although some of the caregivers were already doing tasks such as meal preparation, household chores, and shopping prior to the stroke, many more took on these tasks for the first time after the stroke and were still providing them at T4. Personal care tasks such as bathing, dressing, or helping the person with stroke to get around had never been done by the respondents in this study prior to the stroke. Telephone check-up on a regular basis was another task generated by the onset of stroke. Transportation for the person with stroke was being provided by over half (57\%) of the caregivers at $\mathrm{T} 4$. 
TABLE IV

PERCENTAGE OF CURRENT CAREGIVERS PERFORMING SPECIFIC TASKS FOR PERSON WITH STROKE

\begin{tabular}{|c|c|c|c|c|c|c|c|}
\hline TASK & $\begin{array}{l}\text { Percent } \\
\text { ho do task }\end{array}$ & $\begin{array}{c}\text { \% Same as } \\
\text { before stroke }\end{array}$ & Daily & $\begin{array}{l}\text { 2-6 Times } \\
\text { a week }\end{array}$ & Week ly & $\begin{array}{l}\text { Less than } \\
\text { weekly }\end{array}$ & Never \\
\hline Meal prep & 49 & 20 & 25 & 1 & 3 & 1 & 51 \\
\hline Feeding & 7 & 1 & 5 & 1 & -- & -- & 93 \\
\hline Dressing & 20 & -- & 16 & 3 & 1 & -- & 80 \\
\hline Bathing & 27 & -- & 7 & 14 & 6 & -- & 73 \\
\hline Help walk/move & 8 & -- & 6 & 1 & 1 & -- & 91 \\
\hline Transportation & 57 & 4 & 7 & 28 & 5 & 13 & 43 \\
\hline Shop & 54 & 9 & 1 & 25 & 9 & 10 & 46 \\
\hline $\begin{array}{l}\text { Household } \\
\text { Chores }\end{array}$ & 49 & 12 & 18 & 14 & 5 & -- & 51 \\
\hline $\begin{array}{l}\text { House/yard } \\
\text { ma intenance }\end{array}$ & 35 & 6 & 3 & 4 & 10 & 12 & 65 \\
\hline Supervision & 19 & 1 & 16 & 1 & 1 & -- & 81 \\
\hline Visit & 49 & 4 & 22 & 10 & 10 & 3 & 51 \\
\hline $\begin{array}{l}\text { Pay bills/ } \\
\text { correspondence }\end{array}$ & 59 & 18 & -- & 9 & 6 & 26 & 41 \\
\hline $\begin{array}{l}\text { Phone checkup } \\
\mathrm{a}=\text { persons for } \\
\text { are not inc }\end{array}$ & $\begin{array}{l}\frac{19}{\text { whom the }} \\
\text { cluded in }\end{array}$ & $\begin{array}{l}\text { oke did not ch } \\
\text { percentages } i\end{array}$ & $\begin{array}{l}9 \\
\text { the } \\
\text { ating }\end{array}$ & $\begin{array}{l}5 \\
\text { e and nur } \\
\text { equency }\end{array}$ & $\begin{array}{c}4 \\
\text { task of }\end{array}$ & $\begin{array}{l}1 \\
\text { performe }\end{array}$ & 81 \\
\hline
\end{tabular}




\section{Respite Care}

Thirty-five percent of the primary support persons who were providing care and support for the person with stroke at T4 $(n=81)$ stated that they were not free to come and go at will due to the dependency of the person with stroke. However, only twenty percent of that group indicated that they received respite care from any source in order to have time for themselves. Many of those had respite help less than once a week, and usually from another family member. Most caregivers who had respite support received it from only one source (84\%); the maximum number of sources of respite care was two. Several caregivers indicated that they simply did not go anywhere because the person with stroke would become too upset by either their absence or by having someone else in the house. Some of the respondents mentioned that the persons with stroke object strenuously to what they refer to as "being baby-sat". Respondents who found it easier to stay home than to go through the hassle of having someone else come often mentioned that the "restriction" and "confinement" were the hardest obstacles to overcome in adjusting to the stroke.

Other caregiving responsibilities

Fourteen percent of the caregivers have dependents under age 18. Another twenty-seven percent of the caregivers have non-stroke-related caregiving responsibilities for family members or friends over 80 years of age. 
CHARACTERISTICS OF THE CAREGIVER'S SOCIAL SUPPORT

Perceived availability of help if needed

Only five percent of the caregivers said that no one would be available to help with chores, cooking, transportation, or other tasks if the respondent needed such help. Ninety-five percent mentioned at least one person, with a maximum of nine people mentioned as being available to help if needed. The mean number of persons mentioned was 3 , S.D. $=2.3$, with a range of 0 to 9 . The modal number of persons perceived as available for help was 1. Ward et al. (1984) also found that only 5\% of their sample of 1,185 persons aged 60 and over indicated that they had no-one to turn to for help if it were needed.

Satisfaction with amount of social contact

Fifty-seven percent of the caregivers were very satisfied with the amount of social contact they had at $\mathrm{T} 4$ and $31 \%$ were somewhat satisfied. The remaining eleven percent were either neutral or dissatisfied with the amount of their social contact. Around eightysix percent of the sample studied by Ward et al. (1984) indicated general satisfaction with the amount of contact they had with others, a finding comparable to the results of this study.

Satisfaction with quality of social contact

Seventy percent of the caregivers were very satisfied with the quality of contact they had with others at T4. Twenty-five percent were somewhat satisfied, four percent were neutral, and only one percent was very dissatisfied with the quality of their social 
contact. In a report of satisfaction with quality of social contacts among caregivers of spinal cord injured persons, Schulz et al. (1987) report that sixty percent of those caregivers had high levels of satisfaction with the quality of their social contacts and fifteen percent indicated low levels of satisfaction. The caregivers in this study seem to be somewhat more satisfied with the quality of their social contacts than were the caregivers of spinal cord injured persons.

Reciprocal confiding relationships

Only five percent of the sample had no individual in whom they confided and $14 \%$ of the sample had no person who confided in them. Although some of the caregivers may have had some type of confiding relationship, eighteen percent did not have any reciprocal confiding relationships in their social networks at T4. The mean number of persons with whom the caregivers had confiding relationships was 2.2, S.D. $=1.8$, with a range from 0 to 9 . The modal number of reciprocal confiding relationships was two.

Help from the social support system for household and caregiving needs Questions regarding amount of help received in the past year were asked only of the caregivers who had been caregiving during that year. If the person with stroke had been deceased for more than a year, these particular questions were skipped.

Help from family members. Sixty-six percent of the caregivers reported receiving some help from family members in the care of the person with stroke during the past year. The average number of family 
members who gave assistance to the respondent during the year was 1.8 , S.D. $=2.0$. Silliman et al. (1987) reported that $73 \%$ of caregivers of stroke patients in their study received help from their families in the care of the person with stroke.

The tasks which were shared most often by other family members at T4 were transportation, visiting with the stroke patient, telephone check-up, shopping, and maintenance of the house and yard. The tasks least often shared by family members were feeding, dressing, bathing, paying bills, and general supervision. Fifteen percent of the caregivers at $\mathrm{T} 4$ received some respite time from other family members. Help from friends and neighbors. At T4 forty-five percent of the sample had received help from friends or neighbors in the past year. The average number of friends and neighbors who assisted with caregiving tasks was $1.1, S . D .=2.0$. The most likely assistance for friends and neighbors to provide was to visit with the person with stroke (23\%). Friends and neighbors also assisted with transportation $(8 \%)$, provision of respite for the respondent (8\%) and phone check-up (6\%). These are tasks which have been identified by Cantor (1983) as being most likely to be provided by friends and neighbors. No friends or neighbors helped with the personal care tasks of feeding, bathing, dressing, or mobility assistance.

Help from community or social service agencies. Only sixteen percent of the T4 sample had received assistance for caregiving tasks from community or social service agencies in the past year. Thirteen percent had assistance from one agency, and one percent each had assistance from 2,3 , and 4 agencies. 
CHARACTERISTICS OF THE PERSONS WITH STROKE

Age

The average age of the persons with stroke at T4 was 70.4, S.D. 11.03, ranging from age 40 to age 92 .

Time since onset of first stroke

The average number of months from the first interview, which took place approximately four to six weeks after the onset of the stroke, was $36.5, S . D .=7.5$.

\section{Site of lesion}

Discharge summaries were received for ninety percent of the sample of care-recipients. Forty-seven percent had experienced left cerebral hemisphere strokes, $32 \%$ had right cerebral hemisphere strokes, $9 \%$ had brain stem strokes, and $2 \%$ had other types of strokes.

\section{Additional strokes}

At $14,14 \%$ of the care-recipients were reported by the caregivers as having experienced at least one additional medically diagnosed stroke. Other caregivers commented that they thought the carerecipients had had additional "little" strokes. Although symptoms indicating that these little strokes may have occurred were also noticed by nurses, physical therapists, or doctors themselves, these episodes were not coded as additional strokes unless there had been a specific medical diagnosis indicating the occurrence of additional completed stroke. 
of those who experienced additional medically diagnosed strokes, half had one additional stroke and the other half had two or more strokes. Eleven percent of these care-recipients required hospitalization for the additional stroke(s), and $12 \%$ experienced decreased functioning from the additional stroke(s). Documented medical information was not collected about additional strokes.

\section{Severity of the stroke}

Perceived severity. Nineteen percent of the caregivers reported that the care-recipient had severe to very severe problems at T4 due to stroke, and needed a great deal of help. At the other end of the spectrum, 22\% reported that no physical problems remained from the stroke which required any assistance from another person. The majority (56\%) reported that the care-recipient still had mild to moderate physical problems caused by the stroke which required some help from another person.

Objective severity. Scores from the caregivers' responses on the Barthel Index of Stroke Severity (Mahoney and Barthel, 1965) indicate that at T4 $43 \%$ of the care-recipients have a completely independent level of physical functioning as measured by this index. Mean score on the Barthel Index, for which 100 is the highest score and indicates functional independence, was 86.7 , S.D. $=19.36$. The scores ranged from 35 to 100 .

Communication difficulties due to stroke. Twenty-one percent of the persons with stroke had serious communication problems at T4 due to the stroke. Twelve percent of the caregivers indicated that they 
had difficulty understanding the speech of the person with stroke, and an additional 9\% indicated that the person with stroke could only communicate through sign language or an alphabet board.

Communication patterns often are more easily understood by persons who are in regular contact with the person who has had a stroke than by persons who see the care-recipient less frequently. In comparison to the $12 \%$ of the persons with stroke who could be understood only with difficulty by the caregiver, twice as many (24\%) were very difficult to understand by people who were in less regular contact.

Personality and behavior changes since the stroke. The percentage of the sample of stroke patients who exhibited changes in both negative and positive personality and behavior characteristics due to the stroke is shown in Table $V$. Although somewhat more than half of the sample indicated that the stroke did not affect many of these behaviors in very noticeable ways, there are some striking changes that would conceivably have important impacts on the wellbeing of the caregiver over a long period of time. Particularly noticeable are increases in forgetfulness and poor memory (68\%), confusion and perplexity (54\%), depression and sadness (53\%) and irritability (48\%). The most common decrease in positive behaviors was the decrease in initiative and in the ability or interest in planning (52\%). 
TABLE V

CHANGES SINCE STROKE IN PERSONALITY/BEHAVIOR OF CARE-RECIPIENT AT T4

LESS MORE SAME

\section{NEGATIVE}

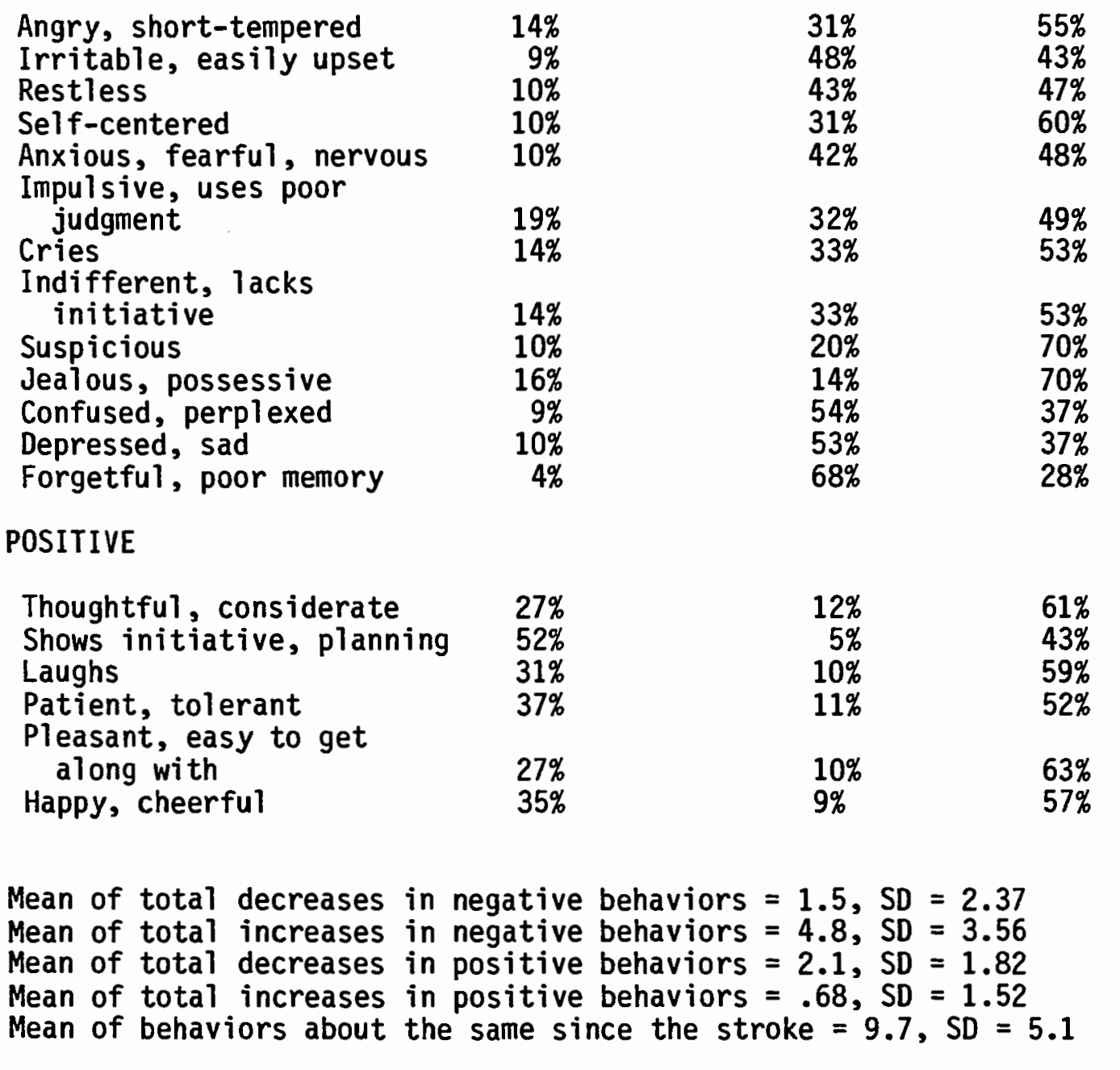

Most of the negative personality/behavior changes which caregivers report happening for the stroke patients in this sample call for some compensatory action from the primary caregiver (such as helping the person with stroke to remember appointments or medication, 
needing to explain things more often or in simpler ways, or becoming the primary person responsible for planning or initiating activities instead of sharing in this role.) Further examination of the effects of changes in these personality/behavior characteristics, and changes over time in specific characteristics which are significantly associated with the outcomes, will be presented in Chapters VI and VII.

General Health Status of Person with Stroke

Eighty-seven percent of the stroke patients for the T4 sample of caregivers were alive at T4. Of those, $78 \%$ were reported by the caregiver as having at least one additional chronic illness besides the residual effects of stroke. Over one-third (40\%) of those had two or more additional chronic illnesses, and $7 \%$ were reported to have four chronic conditions. Twenty-seven percent had been hospitalized for non-stroke related conditions in the year prior to the T4 interview.

Thirty-one percent of the caregivers at T4 reported improved health of the care-recipient since the T3 interview; $32 \%$ felt that the care-recipient's health was about the same as it had been at T3, and $37 \%$ felt that the care-recipient's health was worse since T3.

\section{CAREGIVER STRESS AND BURDEN}

\section{Stress and strain}

At T4 overall physical, emotional, and financial strain were measured on a five point Likert-type scale, ranging from 1 (little or no strain) to 5 (a great deal of strain). Emotional strain ranked the 
highest of the three categories, with almost half of the sample (47\%) reporting that they had experienced very high levels (either 4 or 5) of emotional strain since the stroke. The mean score of emotional strain was 3.4, S.D. $=1.39$. Slightly more than a quarter of the sample (27\%) reported very high levels (either 4 or 5 ) of physical strain in caring for the person with stroke. The mean score for physical strain was 2.6, S.D.= 1.49. Financial strain was quite low for most of the caregivers. Only fourteen percent of the sample reported very high levels of financial strain, and the mean score was .9 , S.D.=1.35. Many caregivers explained that the emotional and physical strain had been very high following the stroke, but had subsided considerably by the T4 interview.

\section{Caregiver Burden}

The Caregiver Burden scale used. at T2, T3, and T4 was adapted from Zarit (1980) for particular use with caregivers of persons who had experienced a stroke (Rau, 1986). It measures aspects of stress such as feeling that one is not doing as much as one can or should for the care-recipient or feeling resentful that the stroke patient makes requests that are over and above what he/she needs. It also measures resentment about other relatives not helping, stress caused by trying to meet the needs of the stroke patient and other responsibilities, and perception that one's personal life and social relationships are being negatively affected by caregiving. In addition, it measures positive aspects of caregiving such as feeling that one is contributing to the well-being of the care-recipient. There are 14 
items on the scale and the maximum possible score is 56 . The mean score at $T 4$ was 17.7, S.D. $=10.3$. Other studies have used different versions of this scale, so direct comparisons of mean scores with other groups of caregivers are not possible. The mean score of perceived burden at T4 was almost identical to the mean score of burden at $T 3 \quad(17.2, S D=10.7)$. Further discussion of changes in perceived burden over time are presented in Chapter VII.

\section{RESPONSES TO STRESS: COPING AND ADJUSTMENT}

\section{Coping strategies}

The design of the T4 study included measurement of coping strategies related to utilization of social network and community resources, to utilization of religious beliefs or resources, and to taking care of oneself through use of planning time for self or using relaxation techniques or exercise. The coping strategies scale, modified from the F-Copes Family Coping Scale (McCubbin, Larsen, and 01 son, 1982), consisted of 15 items with a maximum score of 45 . A low score indicates low levels of utilization of those coping strategies and a high score indicates frequent utilization. The mean for this sample was 11.4 , S.D. $=4.6$, indicating a fairly low use of the coping strategies included in this particular scale.

\section{Adjustment to the stroke}

Caregivers of persons with stroke who were still living at T4 $(n=81)$ were asked how well they felt they had adjusted to the stroke. Fifty-three percent of the caregivers felt they had adjusted to the 
stroke very well, and 38\% felt they had adjusted "pretty well". Nine percent felt they had either not adjusted at all or had adjusted only slightly.

Perception of self as "caregiver"

Respondents were asked if they considered themselves to be "caregivers". Fifty-one percent of the sample indicated that they definitely perceive themselves as caregivers and eleven percent said they "sometimes" do. At least one respondent said emphatically, "You bet I do!" Thirty-eight percent of the respondents did not consider themselves to be caregivers at all.

PSYCHOSOCIAL OUTCOME MEASURES

Depressive symptomatology (CES-D)

The 20-item version of the CES-D scale was used at T4 due to the availability of comparative norms in the literature. With a maximum value of 60 , the mean value for those caregivers currently providing support for the person with stroke was 11.1 , S.D.=9.3. Scores ranged from 0 to 49 , and the median value was 9.0 .

The scores from this sample were somewhat higher than mean scores of general community samples. The HANES Health and Nutrition Examination Study (DHEW, 1979) of almost 5,000 community residents reported a mean score of 8.8 for middle aged respondents and 8.4 for respondents over age 65. Williams (1985) reports a CES-D mean score of 16 for middle aged and elderly persons with chronic illness, and Radloff (1977) reports mean CES-D scores of 24.4 with depressed 
psychiatric patients. Schulz et a1. (1987) report a mean CES-D score of 9.4 for caregivers of spinal cord injured persons, and Van Winkle (1987) reports a CES-D mean score of 12.5 for caregivers of persons with irreversible dementia. The caregivers in this study therefore scored higher on the CES-D than both general community residents and caregivers of spinal cord injured persons but slightly lower than caregivers of persons with irreversible dementia and much lower than either physically $i 11$ persons or psychiatric patients.

A score of 16 is the generally accepted cutoff point indicating high risk of being diagnosed as clinically depressed (Williams, 1985). Twenty-three percent of this sample of caregivers scored 16 or higher on the CES-D. This is somewhat higher than the $17 \%$ reported by Comstock and Helsing (1976) for a general community sample; but considerably less than the $35 \%$ reported by Noh et al. (1984) for a sample of physically disabled persons or the $47 \%$ reported by Williams (1985) for a sample of chronically ill subjects.

Positive Well-Being (POSWB)

The mean score of the POSWB scale for the caregivers at T4 was 4.9, S.D=2.6. The range of scores was 0 to 9 , with a maximum possible value of 9 . The median score was 5.0 .

Negative Well-Being (NEGWB)

The mean score of the NEGWB scale was 3.9, S.D.=2.9. The range of scores was 0 to 13 , with a maximum possible value of 15 . The median score was 4 , and the modal score was also 4 . 
SUMMARY

The descriptive analysis of this sample reveals characteristics which are comparable in many aspects to other caregiver studies. Except for slightly higher levels of education and income, and a somewhat larger percentage of female respondents, this sample is similar in demographic characteristics to large community-based caregiver studies (Stephens and Christianson, 1986; Stone et al., 1987).

The tasks which the caregivers in this study do to assist the care-recipient to carry out activities of daily living are comparable to those reported by Cantor (1980), Stephens and Christianson (1986), and Stone et al. (1987). In addition, the amount of help which the caregiver perceives is available if he/she should need it, the caregiver's general satisfaction with his/her social support system, and the types of assistance which the caregivers receive from others are all comparable to findings reported in several other studies (Cantor, 1980; Ward et a1., 1984; Cohen and Wills, 1985; Stephens and Christianson, 1986; Stone et a1., 1987).

The overall experience of emotional, physical, and financial strain parallels those reported by Stephens and Christianson (1986) in their sample of almost 2,000 community-based caregivers, and supports Brody's (1985) finding that emotional strain is the type of strain most often cited by family caregivers. However, although the mean score for financial strain was low, over ten percent of the sample reported very high levels of financial strain because of the stroke even though those respondents of lower socio-economic status were lost 
to the study at T4. We would therefore hesitate to generalize the findings of this study regarding the experience of low financial strain to other caregiver populations even though it is supported by other studies (e.g. Brody, 1985; Stephens and Christianson, 1986).

The 20-item CES-D mean scores of depressive symptoms in this caregiver sample are somewhat higher than those reported by Schulz et al. (1987) for a sample of caregivers of spinal cord injured persons but somewhat lower than those reported for a sample of caregivers of persons with irreversible dementia (Van Winkle, 1987). The implications of these differences between caregivers of specific disease groups are discussed in Chapter VIII.

The comparability of the characteristics of this study with other caregiver studies provides a foundation for being able to interpret the findings from this study in relation to the population of caregivers as a whole. It is important to remember that the sample for this study was not drawn from a self-selected group of caregivers, but was rather drawn from persons named by the person with stroke as being their primary support person. Even though this sample is remarkably similar to other caregiver studies in demographic and caregiving characteristics, almost forty percent of the respondents do not think of themselves as being "caregivers" for the person with stroke. The implications of this finding are discussed in Chapter VIII.

The following chapter presents the correlational analyses of variables in the model, the results of analyses of variance, and the results from multiple regression procedures. 


\section{CHAPTER VI}

\section{FINDINGS}

\section{INTRODUCTION}

The model for this study as presented in Chapter III postulated that the outcomes of psychological well-being and depressive symptomatology would be affected by certain caregiver characteristics including social support, by characteristics of the stroke, by the experience of perceived stress, and by the caregiver's response to stress and burden. Fifteen research questions were associated with the model and five hypotheses were proposed for testing.

In this chapter the model is tested by examining these relationships as they exist at $\mathrm{T4}$. The results of the analyses are presented as they relate to each component of the model and to the hypotheses and research questions associated with each particular component. First, findings related to the caregiver and caregiving situation are presented. These findings result from analyses of demographic variables as well as social support variables. The findings which relate to the hypothesis that social support will have a buffering role between stress and psychosocial outcomes are also presented in this section. The second section of findings concerns the characteristics of the stroke which are related to the caregiving role. These include a comprehensive index of stroke severity (comprised of communication impairments, negative personality changes, 
and amount of assistance needed), time since onset of stroke, and functional capability of the person with stroke. In addition, analyses of the relationship between caregiver tasks and caregiver outcomes are presented in this section. The third set of results which are reported in this chapter relate to the experience of strain and perceived burden and their effects on the outcome variables of depressive symptomatology and psychological well-being. The fourth set of results are concerned with the caregiver's responses to stress and the hypotheses associated with the buffering effects of coping strategies in conditions of high strain and of high stroke severity. Finally, the results of multiple regression analyses to examine the predictors of long-term depressive symptoms, negative well-being, and positive well-being are presented. The findings associated with change over time in the outcome variables, and with change over time in certain specific variables identified with negative outcomes for the caregiver will be presented in Chapter VII.

\section{CHARACTERISTICS OF THE CAREGIVER}

Effect of death of person with stroke

The first area of interest among the research questions was to compare the outcomes for those caregivers for whom the stroke patient had died by T4 with those who were still in the caregiving role. Ttests and one-way analyses of variance were conducted to analyze these differences. 
The number of respondents who were no longer providing care to the person with stroke because the care-recipient had died was fairly small $(n=12)$, representing about $13 \%$ of the T4 sample. There were no significant differences in depressive symptomatology, positive wel1being, or negative well-being between those respondents who were still in the caregiving role and those who were not. However, for those who were no longer caregiving there was a significant relationship between the number of months since the death of the person with stroke and both depressive symptomatology $(\underline{r}=-.55, p<.05)$ and negative wellbeing $(\underline{r}=-.59, p<.05)$. These relationships indicate that respondents who had experienced the death of the stroke patient more recently were more depressed and had higher negative well-being than those for whom the death had occurred earlier. Because one-third of the deaths occurred within 10 months of the T4 interview, these significant correlations suggest that the finding of no difference between the two groups of respondents may be confounded by the operation of the grief process in some of the respondents.

Since all remaining analyses in this dissertation are concerned with the psychosocial impacts of being in a primary caregiving relationship to a person with stroke, data from respondents who are no longer in the caregiver role because of the death of the person with stroke $(n=12)$ are omitted from further analyses. Further findings are reported only on respondents who are currently in the role of being the primary support person for the person with stroke $(n=81)$. 
Demographic variables

Several demographic variables related to the characteristics of the caregiver were of interest in this study. These were age, income, gender, health status, and relationship to the person with stroke.

Age. As can be seen in Table VI, the age of the caregiver was significantly associated with the outcome of positive well-being $(p<.05)$. The negative direction of the correlation signifies that younger caregivers were more likely to report higher positive wellbeing than older caregivers. The caregiver's age was not associated with the outcomes of depressive symptoms or negative well-being.

Income. Income at the time of the stroke was negatively associated with T4 depressive symptoms and positively associated with positive well-being. It was not significantly associated with negative well-being. A direct measure of change in the respondent's standard of living was included at T4 by asking respondents if they had experienced a change in their standard of living (economically) since the previous interview. If they answered 'yes' to that question, they were asked if their standard of living was now better or worse. Twenty-eight percent of the T4 sample indicated that they had experienced a change in their standard of living. Of these, twothirds said that their standard of living had declined, and one-third felt that it had improved. The direct measure of change in standard of living was significantly correlated with the CES-D scores $(r=-.43$, $\mathrm{p}<.05)$. Those caregivers who reported an improvement in their economic well-being reported fewer depressive symptoms than those 


\section{TABLE VI}

PAIRHISE COIRRELATIONS OF VARIABI.ES IN THE MODEL.

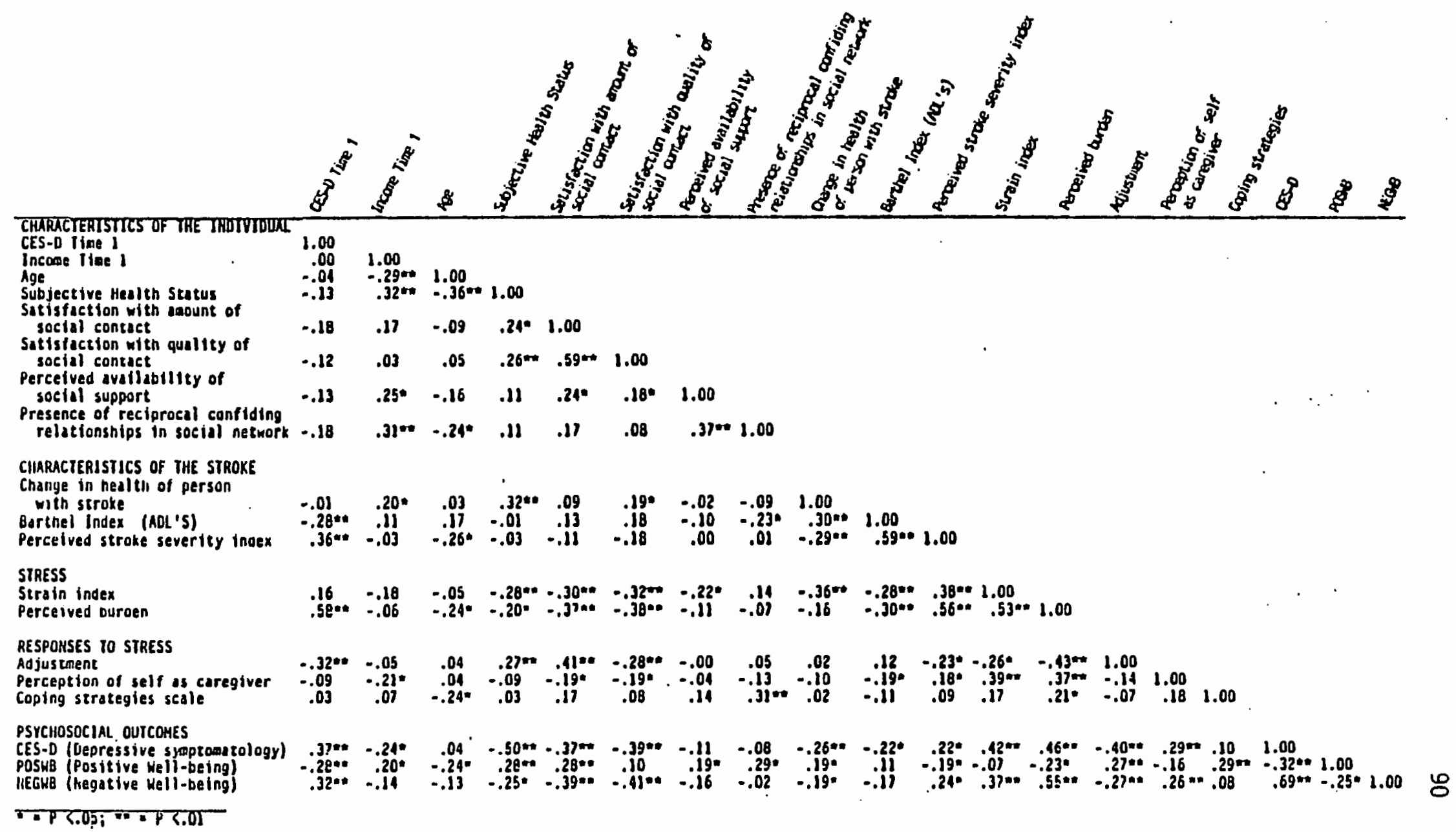


whose economic condition had declined. In addition, caregivers who experienced an improvement in their economic standard of living were significantly less likely to report high levels of perceived burden $(r=-.55, p<.01)$. They were also more likely to have adjusted well to the stroke (stroke $(r=.39, p<.05)$ and less likely to perceive themselves as caregivers $(\underline{r}=-.54, p<.01)$ than caregivers whose economic condition declined between $T 3$ and $T 4$. The findings from this study therefore provide additional support for the large body of literature which finds income to be consistently related to measures of individual well-being (Williams, 1985).

Gender. One-way analysis of variance tests showed no significant gender differences on the CES-D, POSWB or NEGWB measures and no significant differences at the $\mathrm{p}<.05$ level in strain or perceived burden. However, the analysis of variance of gender differences and the strain index approached a level of significance at $p=.07$, indicating that females reported experiencing a slightly higher level of overall strain in caring for the person with stroke than males. The finding of no significant gender differences in perceived burden at T4 is similar to that of Zarit et al. (1986) who found that significant gender differences at $\mathrm{T} 1$ had disappeared by the time of a follow-up interview one year later. However, gender differences on a measure of caregiver stress were found by Horowitz (1985) in her study of sons and daughters as caregivers of elderly parents.

Health status. The subjective health status of the caregiver was significantly correlated with depressive symptomatology, negative well-being, positive well-being, perceived burden, strain, and 
adjustment to the stroke. It is clear that there is an integral relationship between subjective health and both the demands and outcomes of long-term caregiving. Caregivers who perceive their own health as less than optimal at T4 are more likely to report high depressive symptoms, higher negative well-being, lower positive wellbeing, and greater strain and burden than those who feel that their own health is good. However, depression and feelings of burden have also been found to influence a respondent's perception of his or her subjective health status (Williams, 1985; Gallagher et al., 1986).

The objective health status of the caregiver was measured by the total number of chronic illnesses and by the number of chronic illnesses which had occurred after the onset of the stroke. The total number of chronic illnesses was significantly related in a positive direction to depressive symptomatology $(r=.29, p<.01)$ and in a negative direction to positive well-being $(\underline{r}=-.22, p<.05)$. It was not significantly associated with negative well-being, perceived burden, or adjustment to the stroke. As expected, there was a high correlation between total number of chronic illnesses and subjective health status $(\underline{r}=-.55, p<.01)$. In sum, caregivers who had more total chronic illnesses reported significantly higher levels of depressive symptoms and lower levels of both positive well-being and subjective health status than those with fewer chronic illnesses. About sixtyfive percent of the sample reported the onset of at least one chronic illness after the stroke. Additional chronic illnesses were significantly associated with depressive symptoms $(r=.34, p<.01)$ but not with perceived burden or psychological well-being. 
The findings from this study add support to several others which report a high correlation between physical health, chronic illness and depression (Williams, 1985; Westbrook and Viney, 1982; Blazer and Williams, 1980).

Relationship to person with stroke. There was a significant difference between spousal and non-spousal caregivers on the CES-D scores (DF 1,$79 ; F=6.51 ; p=.01$ ), with non-spousal caregivers reporting higher levels of depressive symptomatology than the spousal caregivers. An analysis of variance test was also performed with three groups of caregivers--spouse, adult child, and other. This analysis also resulted in significant differences on the CES-D measure of depressive symptomatology $(D F=2,78, F=3.22, p<.05)$, with adult children scoring significantly higher in depressive symptoms than either spouses or other caregivers. This finding is similar to that of Jones and Vetter (1984) but contrasts with those of Van Winkle (1987) and Benedict et al. (1987) who found no significant differences in depression or morale between spousal and non-spousal caregivers. There were no significant differences between spousal and non-spousal caregivers in the areas of positive or negative well-being, the experience of strain and perceived burden, or in the person with stroke's level of impairment.

Living with versus apart from the person with stroke

The first hypothesis predicted that caregivers who live with the person with stroke will exhibit higher depressive symptoms, higher negative well-being and lower positive well-being than those who live 
apart from the person with stroke. This hypothesis was not supported by the findings in this study. Analyses of variance between caregivers who live with the person with stroke and those who do not indicated no significant differences at the $p<.05$ level between these two groups for the outcome variables of depressive symptoms, positive well-being, or negative well-being, or for the measures of strain or perceived burden. Although some other studies have reported particularly high levels of negative impacts on caregivers who live with the care-recipient (e.g. Soldo and Myllyluoma, 1983; Stephens and Christianson, 1986), significant differences between those who live with the care-recipient and those who do not were not found in this study.

\section{Social Support}

Several hypotheses and research questions were related to the caregiver's social support system. The social support variables included in this study are satisfaction with amount of social support, satisfaction with the quality of social support, perceived availability of support if needed, and the presence of a reciprocal confiding relationship within the support network. These elements of the caregiver's social support system were predicted to have both main effects on depressive symptomatology and psychological well-being, and also to have buffering effects between the condition of high strain or burden and the outcomes of depression and negative well-being.

In presenting the findings related to the caregiver's social support system, correlational analyses are presented first, followed 
by the results of the analyses of variance which were used to test the buffering effects of these variables on the psychosocial outcomes.

Correlations with social support. As can be seen in Table VI, respondents who are satisfied with the amount of social contact they have with others are significantly less likely to experience depressive symptoms, negative well-being, strain, and perceived burden and more likely to report high levels of positive well-being than respondents who are not satisfied with the amount of social contact they have with others. Respondents who are satisfied with the quality of the social contact they have with others are less likely to exhibit depressive symptoms or to report high levels of negative well-being, strain, or perceived burden than those who are less satisfied with the quality of their social contact. These findings are similar to those of Schulz et al. (1987). However, satisfaction with the quality of social contact was not significantly associated with positive wellbeing for this sample.

The perceived availability of social support was significantly associated with positive well-being ( $p<.05)$ but not with negative well-being or with depressive symptoms. Perceived availability of support also was significantly and negatively associated with strain $(p<.01)$. The importance of perceived availability of social support, as opposed to actual support received, is noted by Kessler and McLeod (1985).

The presence of a reciprocal confiding relationship in the caregiver's network was significantly related to positive well-being at the $p<.05$ level. It was not related to negative well-being, 
depressive symptomatology, nor to the experience of strain or perceived burden in this sample.

In sum, the components of the caregiver's social support system which were significantly correlated across strain, perceived burden, depressive symptomatology, and negative well-being were satisfaction with both the amount and quality of social contacts. Satisfaction with the amount of social contact was also significantly correlated with positive well-being. Perceived availability of social support and the presence of a reciprocal confiding relationship in the caregiver's social network were significantly correlated with positive well-being but not with the other outcomes.

The buffering effect of social support. Hypothesis IV (A) predicted that the social support variables would have a buffering effect on depressive symptoms and negative well-being in conditions of high strain and high perceived burden. The hypothesis predicted that caregivers who are satisfied with the amount and quality of their social contacts, who perceive that they have instrumental assistance from their social support system available to them if they need it or who have at least one reciprocal confiding relationship in their social network will be less depressed and have lower levels of negative well-being than those who do not. The hypothesized buffering effects of each of the social support variables on the outcome variables in conditions of high strain and high burden were tested using factorial analyses of variance.

The index of strain was divided into three groups of approximately equal cell size for levels of high strain $(n=26)$, 
moderate strain $(n=30)$, and low strain $(n=25)$. A series of analyses of variance was then performed with the CES-D scores as the dependent variable and with strain and each of the social support variables in turn as the independent variables. The perceived burden scores were also divided into three groups of approximately equal cell size for levels of high, moderate, and low burden. Factorial analyses of variance were conducted to analyze the buffering effects of each of the social support variables on the CES-D scores in conditions of high perceived burden.

In the analysis of the buffering effects of satisfaction with amount of social contact in the condition of high strain, with CES-D scores as the dependent variable, significant main effects were found. Both strain and the caregiver's satisfaction with the amount of social contact were significantly associated with the CES-D scores. However, there were no significant interaction effects. That is, respondents who were very satisfied with the amount of social contact did not have significantly lower depressive symptomatology in conditions of high strain than respondents who were less satisfied with the amount of social contact. Similar results occurred in the analysis of satisfaction with amount of social contact in conditions of high perceived burden with CES-D as the dependent variable: although both perceived burden and satisfaction with the amount of social contact were significantly associated with CES-D by themselves, respondents with high levels of satisfaction with the amount of social contact did 
not have lower levels of depressive symptoms under conditions of high stress than other respondents.

Neither the caregiver's perceived availability of social support nor the presence of a reciprocal confiding relationship in the network had any significant main effects or buffering effects on the CES-D scores. In addition, there were no significant interaction effects between any of the the social support variables and the outcome of negative well-being.

However, significant interaction effects as well as significant main effects were found between satisfaction with quality of social support and CES-D scores in conditions of high strain and also in conditions of high burden. Figure 3 shows the results of an analysis of variance with CES-D scores as the dependent variable by level of strain with satisfaction with quality of social contact (DF=2, $F=3.8$, $p=.03$ ). Figure 4 shows the results of an analysis of variance test with CES-D scores as the dependent variable by level of perceived burden with satisfaction with quality of social contact (DF=2, $F=4.9$, $p=.01$ ). These analyses show that respondents who have high levels of satisfaction with the quality of their social contact maintain relatively low levels of depressive symptoms in conditions of both high strain and high perceived burden. On the other hand, respondents who have low levels of satisfaction with the quality of their social contact show increasing levels of depressive symptomatology with increasing levels of strain and perceived burden. 


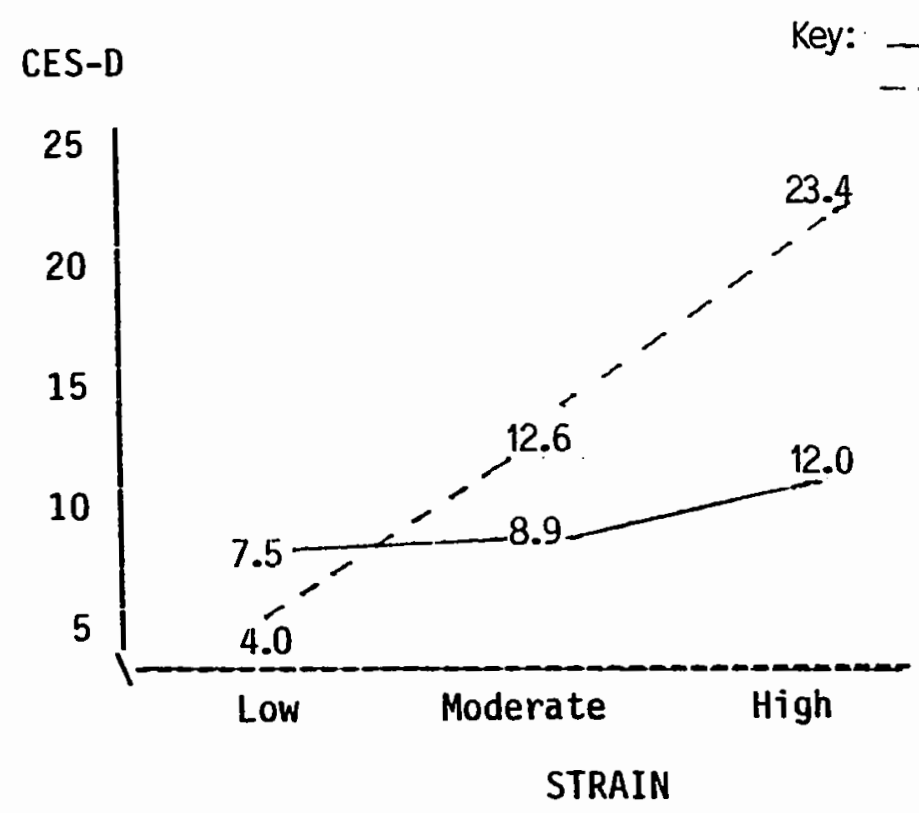

Figure 3. Depression by level of strain with satisfaction with quality of social contact.

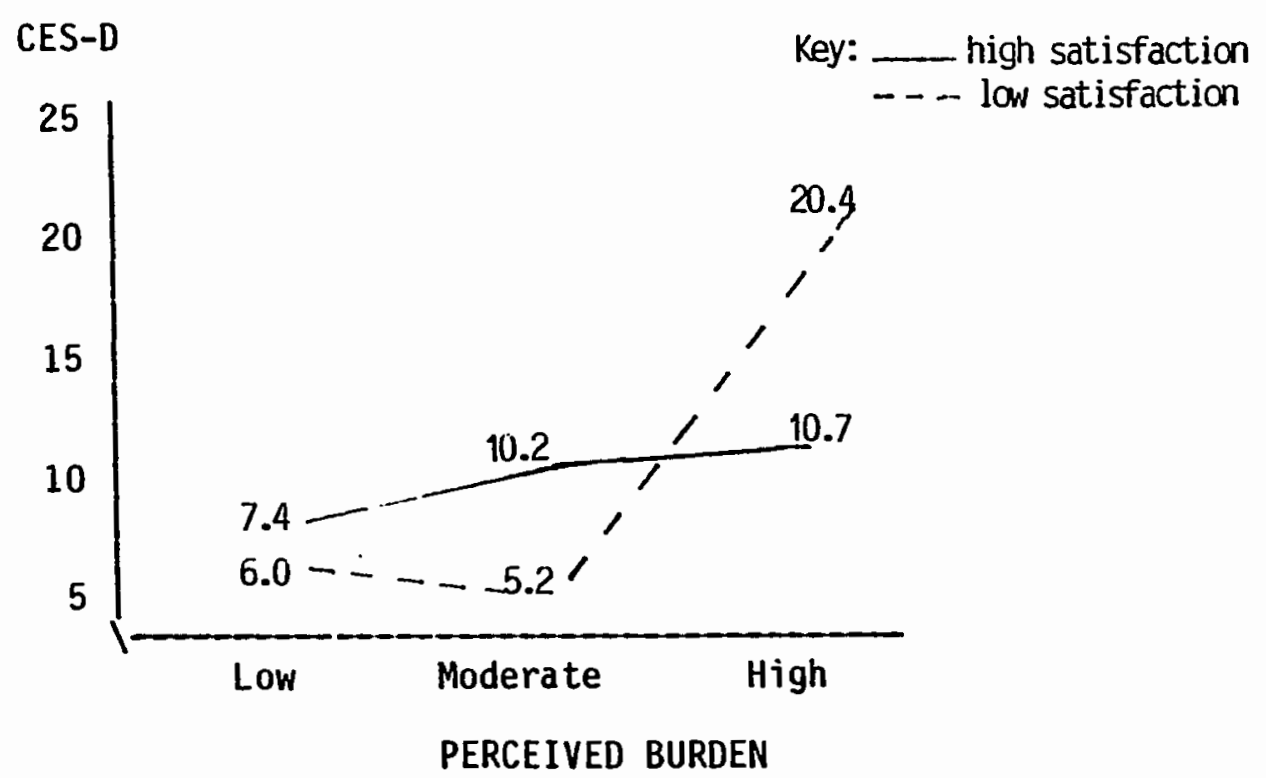

Figure 4. Depression by level of perceived burden with satisfaction with quality of social contact. 


\section{CHARACTERISTICS OF THE STROKE}

One of the research questions of interest in this study concerned the relationship of personality/behavior changes in the person with stroke and the caregiver's experience of burden, depressive symptomatology, and the caregiver's adjustment to the stroke. Table VII shows the correlations of individual items of the measure of personality/behavior changes in the person with stroke with the caregiver's depressive symptomatology (CES-D), perceived burden, and adjustment.

The negative personality/behavior characteristics which were significantly associated with depressive symptoms were 1) angry, short-tempered 2) restless 3) jealous, possessive and 4) forgetful, poor memory. The positive characteristic which was significantly related to depressive symptoms was "pleasant, easy to get along with". The caregivers who reported that the person with stroke was more angry and short-tempered, more restless, and more jealous or possessive since the stroke were significantly more depressed than those who did not report these behavior changes. In addition, caregivers who found that the person with stroke was more pleasant and easy to get along with since the stroke were less depressed than others. A perplexing negative association was found between "forgetful, poor memory" and the caregiver's depressive symptoms, indicating that increases in forgetfulness in the person with stroke were associated with lower depressive symptoms in the caregiver. 
However, increases in forgetfulness were significantly related to higher levels of perceived caregiver burden.

TABLE VII

T4 CORRELATIONS OF INDIVIDUAL PERSONALITY/BEHAVIOR CHANGES

IN THE STROKE PATIENT

WITH CAREGIVER SCORES FOR CES-D, BURDEN AND ADJUSTMENT

\section{CES-D BURDEN ADJUSTMENT}

\section{NEGATIVE}

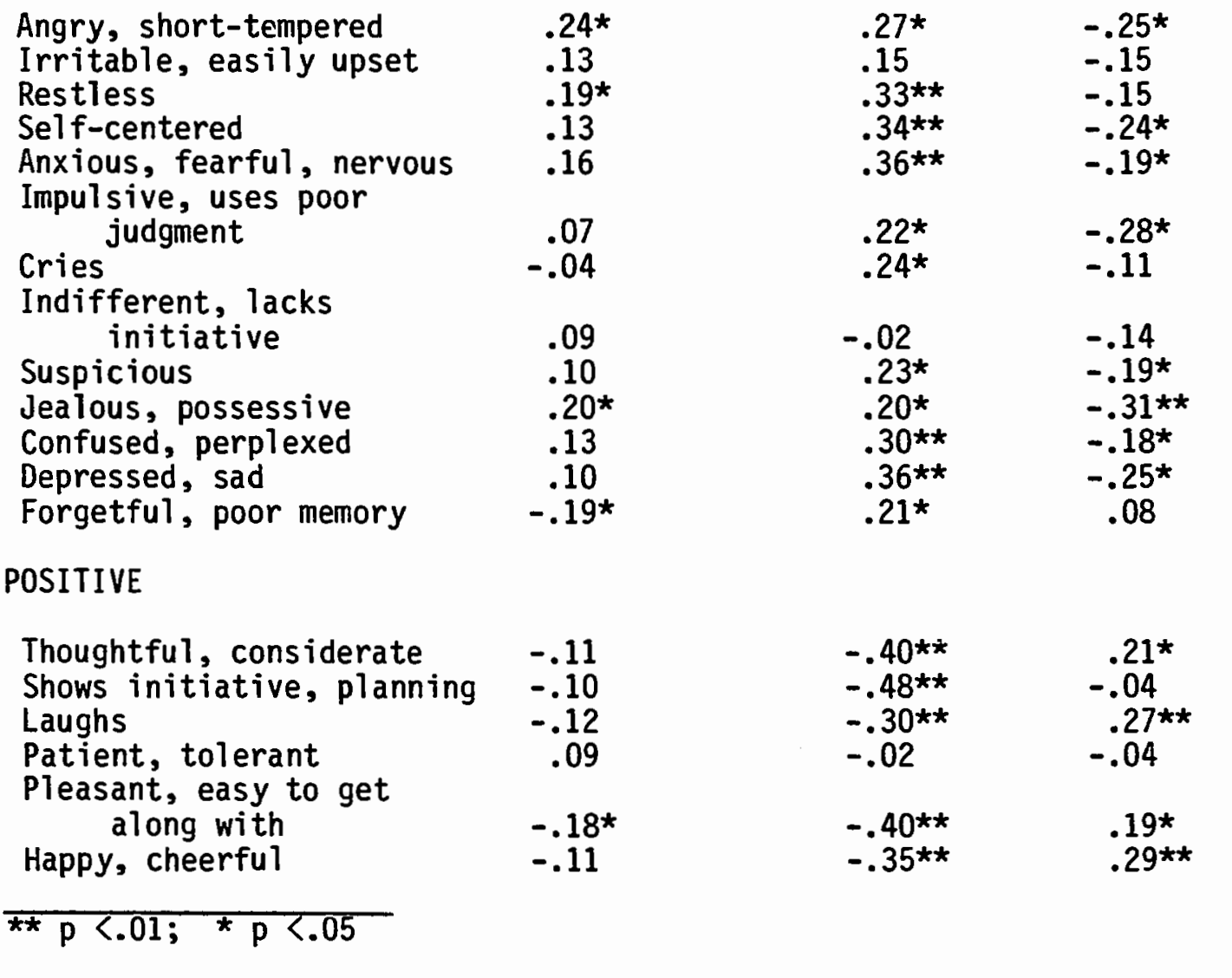

As can be seen in Table VII, many more of the reported stroke-related changes in personality and behavior characteristics of 
the person with stroke were significantly associated with perceived burden and adjustment to the stroke than were associated with depressive symptoms. Caregivers who reported that the person with stroke was more cheerful, more pleasant and easy to get along with, laughed more and was more thoughtful since the stroke were significantly more well adjusted and reported significantly lower levels of perceived burden than caregivers of persons who exhibited decreases in these characteristics.

Caregivers who reported stroke-related increases in the characteristics of anger, self-centeredness, anxiety, impuisiveness, suspicion, jealousy, confusion, and depression in the person with stroke were significantly more burdened and less well adjusted than those who did not report increases in these characteristics. In addition, increased restlessness in the person with stroke, increases in crying, and decreases in initiative and planning were significantly associated with perceived caregiver burden but had no significant relationship to caregiver adjustment to the stroke.

Another question related to the stroke concerned the relationship of caregiving tasks to the psychosocial outcomes and to the experience of burden. Neither the total number of tasks nor the total frequency of task provision were significantly associated with depressive symptoms or with positive or negative well-being. However, both the total number of tasks and the total frequency of tasks were significantly associated with perceived burden $(\underline{r}=.36, p<.01, \underline{r}=.41$, $p<.01$, respectively), with the level of functional capacity of the 
person with stroke ( $r=-.39, p<.01$ and $\underline{r}=-.47, p<.01$, respectively), and with perception of self as a "caregiver" ( $r=.29, p<.01$ for each).

A third research question associated with the stroke asked if there was a significant relationship between the site of lesion and the caregiver outcomes. There were no significant correlations between site of lesion and any of the outcome variables.

Hypothesis II predicted that the long-term severity of the stroke, which includes the amount of assistance needed by the person with stroke, the negative personality/behavior changes which result from the stroke, and the stroke-related communication impairments would be significantly related to the well-being outcomes. Specifically, it was hypothesized that a comprehensive index of stroke severity would be significantly related in a positive direction to perceived stress (strain and perceived burden), depressive symptomatology and negative well-being, and significantly related in a negative direction to positive well-being.

Al1 elements of Hypothesis II were supported by the findings. As can be seen in Table VI, the correlations of the stroke severity index with caregiver strain and perceived burden were significant at the $p<.01$ level, and the correlations with the psychosocial outcomes of depressive symptomatology, positive well-being and negative wellbeing were each significant at the $p<.05$ level. All correlations were in the hypothesized directions. The finding of a significant correlation between the stroke severity index and caregiver burden contrasts with Zarit et. al's (1986) report of no significant correlation between the level of the care-recipient's impairment and 
caregiver burden for caregivers of Alzheimer's patients. In addition, the findings from this study also contrast with those of Wade et al. (1986), who found that although the stroke patient's physical disability (as measured by the Barthel Index) was significantly related to caregiver depression three weeks after the stroke and one year after the stroke, it had no significant relationship to caregiver depression after two years.

\section{PERCEIVED STRESS}

The experience of strain and perceived burden are the variables included in this component of the model. The index of strain is a composite of emotional strain, physical strain, and financial strain. The construction of this index was presented in Chapter IV. The measure of perceived burden was adapted by Rau (1986) from Zarit et al. (1980) for use with caregivers of persons with stroke. Research questions asked about specific items in the caregiver burden scale which might be particularly related to long-term psychosocial outcomes. The correlations of the individual caregiver burden scale items are shown in Table VIII.

Six items were significantly correlated at the $p<.01$ level with both high depressive symptoms (CES-D) and low caregiver adjustment. These were 1) feeling that the person with stroke requests more of the caregiver than necessary, 2) not having enough time to oneself, 3) feeling stressed between trying to give to the person with stroke and other responsibilities, 4) feeling that one's relationship with family 
TABLE VIII

T4 CORRELATIONS OF INDIVIDUAL ITEMS

ON THE CAREGIVER BURDEN SCALE

WITH CAREGIVER SCORES FOR CES-D AND ADJUSTMENT

CES-D ADJUSTMENT

1. I feel I don't do as much for the stroke patient as I could or should

$\begin{array}{ll}.20 \star & -.02{ }^{*} \\ .07 & .00 \\ -.03 & -.15 \\ .32 \star \star & -.31 \star \star \\ .35 * \star & -.48 \star \star\end{array}$

6. I feel resentful of other relatives who could but who don't do things for the stroke patient

$.35^{\star \star}-.15$

7. I feel stressed between trying to give both to the stroke patient and to other responsibilitie s

$.33^{\star *}-.28 * \star$

8. I feel the stroke patient affects my relationships with family/friends in a negative way

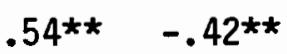

9. I feel useful in my interactions with the stroke patient (high score=never)

$.35 * \star-.06$

10. I feel that my health has suffered because of my involvement with the stroke patient

$.59 * *-.40 * *$

11. I feel that my social life has suffered because of my involvement with the stroke patient

$.32 * \star-.46 \star \star$

12. I feel that the stroke patient doesn't appreciate what I do for him/her as much as I would like

13. I feel that the stroke patient expects me to take care of him/her as if I were the only one he/she could depend on

$.07-.38^{\star \star}$

14. I feel embarrassed over the stroke patient's behavior or condition 
and friends has been affected in a negative way by the person with stroke, 5) feeling that one's health has suffered because of caregiving for the person with stroke, and 6) feeling that one's social life has been negatively affected by the caregiving role. Some studies which have reported individual items of caregiver burden have found similar relationships (Pratt et al., 1985; 1987).

Hypothesis III predicted that both the strain index and perceived burden would be significantly correlated in a positive direction with depressive symptomatology and negative well-being and significantly correlated in a negative direction with positive wellbeing. The Pearson correlation coefficients for these variables are shown in Table VI. The index of strain and the perceived burden scale were both significantly correlated with depressive symptomatology and negative well-being in the predicted directions. Although the perceived burden scale was significantly correlated in a negative direction with positive well-being, the index of strain was not significantly related to positive well-being. The findings related to this hypothesis support the strong relationship between perceived burden and strain with negative psychosocial outcomes for the caregiver. However, although lower perceived burden is significantly associated with positive well-being for the caregiver, lower levels of strain are not significantly related to the caregiver's positive well-being. 
RESPONSES TO STRESS

Three caregiver responses to stress were included in the model for this study. These were 1) use of coping strategies 2) adjustment to the stroke and 3) perception of self as caregiver. Hypothesis IV (B) predicted that the development of coping strategies would serve as a buffer between the condition of high strain and high perceived burden and the outcomes of depressive symptomatology and negative well-being. It was also predicted that coping strategies would interact with strain and perceived burden in predicting positive wellbeing.

The measure of coping strategies was significantly associated with positive well-being but not with depressive symptomatology or negative well-being. It was negatively associated with age, suggesting that younger people were more likely to use coping strategies in response to the stress of caregiving. The positive association between coping strategies and the presence of reciprocal confiding relationships in the network may reflect the specific items on the coping strategies scale which measured the frequency of sharing concerns with relatives or close friends. Coping was also positively associated with the caregiver burden score, signifying that perhaps the respondents who feel more burdened may use more coping strategies than those who feel less burdened.

The hypothesized buffering effects of coping strategies on the depressive symptomatology and negative well-being were not supported by the findings in this study. The lack of significant correlations 
between coping strategies and either depressive symptoms or negative well-being precluded the finding of significant main effects or significant interactions through analysis of variance. The results of analysis of variance tests with coping strategies dichotomized at the median score and positive well-being as the dependent variable showed no significant main or interaction effects of coping on positive wellbeing.

"Adjustment to the stroke" and "perception of self as a 'caregiver'" were the remaining variables in the responses to stress component of the model. A search of the literature revealed no other examinations to date of these particular variables in relation to caregiver outcomes. Two sets of research questions were associated with each of these variables. The first was interest in knowing if the respondents perceive themselves as 'caregivers', and whether or not an association exists between perceiving oneself as a caregiver and the psychosocial outcomes of caregiving. In addition, what association exists between perception of self as a caregiver and the experiences of strain, burden, and adjustment to stroke?

Almost two-thirds of the sample (62\%) considered themselves to be "caregivers". As illustrated in Table VI, perception of self as caregiver was significantly correlated with the outcome of depressive symptomatology but not with either positive or negative well-being. The positive direction of the correlation indicates that respondents who perceived themselves as caregivers were more likely to have significantly higher scores on the measure of depressive symptomatology than those who did not see themselves as caregivers. 
This variable was also significantly associated in a positive direction with the index of stroke severity, strain, and perceived burden. These associations suggest that respondents who considered themselves to be caregivers may have been caring for persons who had more severe long-term negative effects from the stroke than the other care-recipients. These respondents also experienced high levels of strain and felt considerably burdened by the caregiving role. In addition, perception of self as caregiver was significantly associated with lower income and lower levels of satisfaction with the amount and quality of their social contacts.

The second set of research questions associated with responses to stress concerned the role which the caregiver's adjustment plays in the long-term experience of strain, burden, and psychosocial outcomes. Are there differences in the outcomes at $\mathrm{T} 4$ between respondents who feel they have adjusted well to the stroke and those who have not adjusted well?

Table IX presents the correlations of adjustment with the outcomes and the intervening variables of strain and perceived burden. Adjustment to the stroke was significantly correlated in a negative direction with depressive symptomatology and negative well-being. It was correlated significantly in a positive direction with positive well-being. These correlations, all significant at the $p<.01$ level, indicate that high levels of adjustment are significantly related to long-term psychological well-being in the caregiver. Adjustment was 
also significantly related to lower burden, strain, and stroke severity.

It was of further interest to inquire if the caregiver's adjustment to the stroke would act as a buffer between conditions of high perceived stroke severity or high strain and the outcomes of depressive symptomatology and/or well-being. In separate factorial

TABLE IX

T4 CORRELATIONS OF CAREGIVER ADJUSTMENT WITH STROKE VARIABLES, PERCEIVED STRESS, AND OUTCOMES

ADJUSTMENT TO STROKE

STROKE VARIABLES

Barthel index

Perceived stroke severity index

.12

$-.23^{*}$

PERCEIVED STRESS

Strain index

Perceived burden

$-.26 *$

$-.43 \star \star$

OUTCOMES

CES-D

NEGWB

$-.40 \star \star$

POSWB

$-.27 \star \star$ $.27 \star \star$

$\star \star=p<.01$

$\star=p<.05$

analyses of variance, adjustment (high, low) was tested with strain (dichotomized at the median score to produce high and low levels) and again with the index of stroke severity (dichotomized at the median to produce high and low levels) with CES-D scores as the dependent variable in each analysis. Although the results showed significant 
main effects for the individual variables on the CES-D scores there were no interaction effects of adjustment between either the conditions of high strain or high stroke severity and the depressive symp tomatology scores.

\section{RESULTS OF MULTIPLE REGRESSION ANALYSIS}

The final stage of analysis reported in this chapter is the analysis of predictors of long-term depressive symptomatology and psychological well-being through the application of multiple regression analysis. Several hierarchical regressions were performed to examine the relative contributions of predictor variables to T4 outcomes of depressive symptomatology, positive well-being, and negative well-being.

The first procedure in each of the regression analyses was to determine the order of entry of the blocks of variables containing the characteristics of the caregiver versus the characteristics of the stroke. The literature generally supports the greater importance of caregiver variables over variables related to the characteristics of the illness in predicting caregiver outcomes (Zarit et al.,1986; Morycz, 1985). In order to test the relative importance of these two blocks of variables with this particular sample individual regressions were performed with those blocks regressed separately (after the control variables of age and income) on each of the outcome variables. For example, the first regression analysis which was performed with CES-D as the dependent variable included age and income as control variables and the remaining characteristics of the 
caregiver were entered as the next block of independent variables. Then a new regression analysis was performed with CES-D as the dependent variable, age and income as control variables, and the characteristics of the stroke entered as the next block of independent variables. This procedure was performed next with NEGWB as the dependent variable, and aga in with POSWB as the dependent variable. In each case, the characteristics of the caregiver contributed more to the explanation of variance in the dependent variable than did the characteristics of the stroke. Therefore, in each of the hierarchical regression analyses the characteristics of the caregiver were entered after the control variables and were followed by entry of the block of stroke-related variables.

The multiple regression analys is for each outcome (CES-D, NEGWB, and POSWB) tested predictors at T4 without controlling for $\mathrm{Tl}$ levels of depressive symptoms or for $\mathrm{Tl}$ levels of positive or negative wellbeing. This was done in order to examine long-term predictors of each of the outcomes.

The variables were selected for inclusion in the regression analyses on the basis of their significant correlation with each specific outcome variable. Early regression analyses indicated the potential presence of multicollinearity among some of the variables. Separate regression analyses were performed to identify the collinear variables. The analyses showed collinearity between satisfaction with amount of social contact and satisfaction with quality of social contact. Therefore, these two variables were combined into a social support satisfaction index for use in the regression analyses. A 
Cronbach's alpha test for internal consistency of this index resulted in an acceptable alpha $(a=.75)$. High collinearity was also found between the change in health status of the person with stroke and the Barthel index of functional capability. Since the health status measure is a one-item response and the Barthel index is a validated and reliable eleven-item scale which also captures the construct of health status, the Barthel index was chosen as the better predictor variable and the health status measure was dropped from the regression analyses.

Tables $X$ to XII present the results of the regression analyses. The design of the tables follows a specific pattern. Each column of the table (labeled Model $1, \operatorname{Model}_{2}$, etc.) shows the hierarchical regression of variables in the model as they are entered hierarchically in successive blocks. In each table Model 1 includes the control variables, Model 2 includes the control variables plus characteristics of the caregiver, Model 3 includes the block of stroke-related variables as the next entry, Model 4 includes the subsequent entry of the block of perceived stress variables, and Model 5 includes the complete regression with the addition of the block of variables related to responses to stress. The $R^{2}, R^{2}$ change, $F$, and significance of $F$ are reported at the bottom of each column.

The following sections present the results of the regression analyses for each of the outcome variables. 
CES-D as the dependent variable

Table $X$ shows the results of hierarchical regression analysis for CES-D at T4. Forty-six percent of the variance in the CES-D scores at T4 is explained by the variables in the model. The block of variables which adds the most to the explanation of variance in depressive symptoms is that which contains the characteristics of the caregiver. This block of variables explains thirty-one percent of the variance in CES-D after the effects of age and income have been controlled. When objective health status was entered as a control variable there was less than one percent change in the regression equation and no change in the significance level of subjective health as a predictor of depressive symptoms. Because of its lack of impact on the model, objective health was not included in the equation in order to economize on the number of variables in the model.

The block of variables containing significant stroke-related variables explains two percent of the variance in CES-D. Caregiver stress explains five percent in the variance of T4 depressive symptoms, and the caregiver's responses to stress provide the explanation two percent of the variance in CES-D. The analys is from this study therefore adds further support to the growing body of literature which has reported that characteristics of the caregiver are more important in explaining caregiver outcomes than are characteristics of the care-recipient (Zarit et al., 1986; Morycz, 1985; Borgatta and Montgomery, 1987). Of all the characteristics of the caregiver included in the model the respondent's subjective health status is the most significant predictor of depressive symptoms at T4. 
TABLE X

\begin{tabular}{|c|c|c|c|c|c|c|c|c|c|c|}
\hline \multirow{2}{*}{ Dependent Variable: CES-D at Time 4} & \multicolumn{10}{|c|}{ HIERARCHICAL REGRESSION FOR CES-D AT T4 } \\
\hline & B & Beta & B & Beta & B & Beta & B & Beta & B & Beta \\
\hline $\begin{array}{l}\text { CONTROL VARIABLES } \\
\text { Age } \\
\text { Time } 1 \text { Income }\end{array}$ & $\begin{array}{l}-.02 \\
-.13\end{array}$ & $\begin{array}{l}-.03 \\
-.25^{\star}\end{array}$ & $\begin{array}{l}-.11 \\
-.06\end{array}$ & $\begin{array}{l}-.15 \\
-.11\end{array}$ & $\begin{array}{l}-.07 \\
-.05\end{array}$ & $\begin{array}{l}-.10 \\
-.09\end{array}$ & $\begin{array}{l}-.04 \\
-.04\end{array}$ & $\begin{array}{l}-.05 \\
-.07\end{array}$ & $\begin{array}{l}-.05 \\
-.04\end{array}$ & $\begin{array}{l}-.07 \\
-.08\end{array}$ \\
\hline $\begin{array}{l}\text { CHARACTERISTICS OF CAREGIVER } \\
\text { Satisfaction with } \\
\text { social support (index) } \\
\text { Subjective health status }\end{array}$ & & & $\begin{array}{l}-1.53 \\
-3.71\end{array}$ & $\begin{array}{l}-.29 \star \star \\
-.44 \star \star\end{array}$ & $\begin{array}{l}-1.38 \\
-3.68\end{array}$ & $\begin{array}{l}-.27 \star \star \\
-.44 \star \star\end{array}$ & -.86 & $\begin{array}{l}-.17 \\
-.38 \star \star\end{array}$ & $\begin{array}{r}-.69 \\
-3.04\end{array}$ & $\begin{array}{l}-.13 \\
-.36 \star \star\end{array}$ \\
\hline $\begin{array}{l}\text { CHARACTERISTICS OF STROKE CONDUCIVE } \\
\text { TO CAREGIVER STRESS } \\
\text { Barthel index of functional capacit } \\
\text { Perceived severity of stroke index }\end{array}$ & & & & & $\begin{array}{r}-.05 \\
.26\end{array}$ & $\begin{array}{r}-.11 \\
.08\end{array}$ & $\begin{array}{l}-.06 \\
-.25\end{array}$ & $\begin{array}{l}-.12 \\
-.07\end{array}$ & $\begin{array}{l}-.05 \\
-.24\end{array}$ & $\begin{array}{l}-.11 \\
-.07\end{array}$ \\
\hline $\begin{array}{l}\text { CAREGIVER STRESS } \\
\text { Strain index } \\
\text { Perceived burden }\end{array}$ & & & & & & & $\begin{array}{l}.44 \\
.22\end{array}$ & $\begin{array}{l}.10 \\
.24\end{array}$ & $\begin{array}{l}.35 \\
.16\end{array}$ & $\begin{array}{l}.08 \\
.17\end{array}$ \\
\hline $\begin{array}{l}\text { RESPONSE TO STRESS } \\
\text { Adjustment } \\
\text { Perception of self as caregiver }\end{array}$ & & & & & & & & & $\begin{array}{r}-2.66 \\
.88\end{array}$ & $\begin{array}{r}-.14 \\
.09\end{array}$ \\
\hline $\begin{array}{l}R^{2} \\
R^{2} \text { Change }\end{array}$ & & $\begin{array}{l}.06 \\
.06\end{array}$ & & $\begin{array}{l}37 \\
31\end{array}$ & & $\begin{array}{l}.39 \\
.02\end{array}$ & & $\begin{array}{l}.44 \\
.05\end{array}$ & & \\
\hline$=0<01 \% \star=0<05$ & $\begin{array}{l}F= \\
p=\end{array}$ & $\begin{array}{l}2.32 \\
.10\end{array}$ & $\begin{array}{l}F= \\
p=\end{array}$ & $\begin{array}{r}10.4 \\
.00\end{array}$ & & $\begin{array}{l}=7.46 \\
=.00\end{array}$ & $p$ & $\begin{array}{l}6.67 \\
=.00\end{array}$ & $\begin{array}{l}F= \\
p=\end{array}$ & $\begin{array}{l}.63 \\
00\end{array}$ \\
\hline
\end{tabular}


NEGWB as the dependent variable

Table XI illustrates the regression analysis of variables in the model which were significantly correlated with NEGWB at T4 regressed on the NEGWB T4 scores. A total of thirty-seven percent of the variance in negative well-being at $T 4$ is explained by the variables in the model. The characteristics of the caregiver explain twenty-one percent of the variance in T4 NEGWB scores. An additional three percent is explained by the characteristics of the stroke. Caregiver stress variables add another 13 percent to the understanding of variance in T4 negative well-being, but response to stress explains less than an additional one percent of the variance in those scores. The most significant predictor of long-term negative well-being is perceived caregiver burden. An additional significant predictor of long-term negative well-being is the caregiver's satisfaction with the amount and quality of his/her social support. 
TABLE XI

\begin{tabular}{|c|c|c|c|c|c|c|c|c|c|c|}
\hline Dependent Variable: NEGWB at Time 4 & \multicolumn{8}{|c|}{ HIERARCHICAL REGRESSION FOR NEGWB AT T4 } & & $\mathrm{del}_{5}$ \\
\hline $\begin{array}{l}\text { CONTROL VARIABLES } \\
\text { Age } \\
\text { Time } 1 \text { Income } 1\end{array}$ & B & Beta & B & Beta & B & Beta & B & Beta & B & Beta \\
\hline $\begin{array}{l}\text { CHARACTERISTICS OF CAREGIVER } \\
\text { Satisfaction with } \\
\text { social support (index) } \\
\text { Subjective health status }\end{array}$ & & & $\begin{array}{l}-.66 \\
-.36\end{array}$ & $\begin{array}{l}-.40 \star \star \\
-.14\end{array}$ & $\begin{array}{l}-.61 \\
-.33\end{array}$ & $\begin{array}{l}-.38 * * \\
-.12\end{array}$ & $\begin{array}{l}-.36 \\
-.21\end{array}$ & $\begin{array}{l}-.22 \star \\
-.08\end{array}$ & $\begin{array}{l}-.37 \\
-.23\end{array}$ & $\begin{array}{l}-.23^{\star} \\
-.09\end{array}$ \\
\hline $\begin{array}{l}\text { CHARACTERISTICS OF STROKE CONDUCIVE } \\
\text { TO CAREGIVER STRESS } \\
\text { Perceived severity of stroke index }\end{array}$ & & & & & .17 & .16 & -.08 & -.07 & -.07 & -.07 \\
\hline $\begin{array}{l}\text { CAREGIVER STRESS } \\
\text { Strain index } \\
\text { Perceived burden }\end{array}$ & & & & & & & $\begin{array}{l}.08 \\
.12\end{array}$ & $\begin{array}{l}.06 \\
.44 \star \star\end{array}$ & $\begin{array}{l}.08 \\
.13\end{array}$ & .07 \\
\hline $\begin{array}{l}\text { RESPONSE TO STRESS } \\
\text { Adjus tment }\end{array}$ & & & & & & & & & .23 & .04 \\
\hline $\begin{array}{l}R_{2}^{2} \\
R^{2} \text { Change }\end{array}$ & & & $\begin{array}{l}F= \\
p=\end{array}$ & $\begin{array}{l}21 \\
21 \\
10.48 \\
.00\end{array}$ & & $\begin{aligned} & .24 \\
& .03 \\
&= 8.13 \\
&= .00\end{aligned}$ & $\begin{array}{l}F \\
p\end{array}$ & $\begin{array}{l}.37 \\
.13 \\
=8.66 \\
=.00\end{array}$ & $\begin{array}{l}< \\
F= \\
p=\end{array}$ & $\begin{array}{l}37 \\
01 \\
7.16 \\
.00\end{array}$ \\
\hline
\end{tabular}

1. Not entered in the regression due to lack of significant correlation with NEGWB at T4 $\star *=p<.01 ; *=p<.05$ 
POSWB as the dependent variable

Table XII shows the results of multiple regression with positive well-being (POSWB) at T4 as the dependent variable. In the regression of T4 variables on POSWB a total of thirty percent of the variance is explained. After controlling for the effects of age and income on POSWB, the characteristics of the caregiver explain ten percent of the variance in POSWB at T4. The characteristics of the stroke explain another four percent, and caregiver stress explains another one percent. Responses to stress, which include adjustment to stroke and use of coping strategies add a final eight percent to the explanation of variance in positive well-being at T4. The significant predictor of positive well-being for this sample is the development of coping strategies.

HYPOTHESES SUPPORTED AND NOT SUPPORTED BY THE FINDINGS

Table XIII illustrates the extent to which the hypotheses which were presented in Chapter III are supported by the findings in this study. Support was not found for the hypothesis that caregivers who lived with the person with stroke would be more adversely affected over time than those who lived apart from the care-recipient.

Support was found for the hypothesized relationship of the comprehensive stroke severity index to the caregiver's experience of strain and burden as well as to the three outcome measures of psychological well-being. Support was also found for the hypothesized relationship of the experience of strain and burden to the outcome measures. 
TABLE XII

\begin{tabular}{|c|c|c|c|c|c|c|c|c|c|c|}
\hline \multirow{2}{*}{ Dependent Variable: POSWB at Time 4} & \multicolumn{10}{|c|}{ HIERARCHICAL REGRESSION FOR POSWB AT T4 } \\
\hline & B & Beta & $\mathrm{B}$ & Beta & $\mathrm{B}$ & Beta & B & Beta & $\mathrm{B}$ & Beta \\
\hline $\begin{array}{l}\text { CONTROL VARIABLES } \\
\text { Age } \\
\text { Time } 1 \text { Income }\end{array}$ & $\begin{array}{r}-.04 \\
.02\end{array}$ & $\begin{array}{r}-.20 \\
.14\end{array}$ & $\begin{array}{r}-.02 \\
.00\end{array}$ & $\begin{array}{r}-.12 \\
.02\end{array}$ & $\begin{array}{r}-.04 \\
.00\end{array}$ & $\begin{array}{r}-.19 \\
.00\end{array}$ & $\begin{array}{r}-.04 \\
.00\end{array}$ & $\begin{array}{r}-.21 \\
.00\end{array}$ & $\begin{array}{r}-.03 \\
.00\end{array}$ & $\begin{array}{r}-.17 \\
.05\end{array}$ \\
\hline $\begin{array}{l}\text { CHARACTERISTICS OF CAREGIVER } \\
\text { Satisfaction with } \\
\text { social support (index) } \\
\text { Subjective heal th status } \\
\text { Perceived availabil ity of soc. supp } \\
\text { Reciprocal confiding rel. in networ }\end{array}$ & $\begin{array}{l}\text { port } \\
\text { rk }\end{array}$ & & $\begin{array}{l}.18 \\
.37 \\
.05 \\
.28\end{array}$ & $\begin{array}{l}.13 \\
.16 \\
.05 \\
.20\end{array}$ & $\begin{array}{l}.14 \\
.33 \\
.06 \\
.28\end{array}$ & $\begin{array}{l}.10 \\
.14 \\
.05 \\
.14\end{array}$ & $\begin{array}{l}.09 \\
.28 \\
.05 \\
.27\end{array}$ & $\begin{array}{l}.06 \\
.12 \\
.05 \\
.19\end{array}$ & $\begin{array}{r}-.07 \\
.22 \\
.07 \\
.15\end{array}$ & $\begin{array}{r}-.05 \\
.09 \\
.06 \\
.11\end{array}$ \\
\hline $\begin{array}{l}\text { CHARACTERISTICS OF STROKE CONDUCIVE } \\
\text { TO CAREGIVER STRESS } \\
\text { Perceived severity of stroke index }\end{array}$ & & & & & -.21 & $-.22 *$ & -.16 & -.16 & -.13 & -.13 \\
\hline $\begin{array}{l}\text { CAREGIVER STRESS } \\
\text { Perceived burden }\end{array}$ & & & & & & & -.03 & -.12 & -.04 & -.15 \\
\hline $\begin{array}{l}\text { RESPONSE TO STRESS } \\
\text { Adjustment } \\
\text { Coping strategies }\end{array}$ & & & & & & & & & $\begin{array}{r}1.01 \\
.15\end{array}$ & $\begin{array}{l}.20 \\
.26^{\star}\end{array}$ \\
\hline $\begin{array}{l}\mathrm{R}^{2} \\
\mathrm{R}^{2} \text { Change }\end{array}$ & & $\begin{array}{l}.07 \\
.07\end{array}$ & & $\begin{array}{l}17 \\
10\end{array}$ & & $\begin{array}{l}.21 \\
.04\end{array}$ & & $\begin{array}{l}.22 \\
.01\end{array}$ & & $\begin{array}{l}.30 \\
.08\end{array}$ \\
\hline$\star \star=0<01: \quad *=0<.05$ & $\begin{array}{l}F= \\
p=\end{array}$ & $\begin{array}{l}2.99 \\
.05\end{array}$ & $\begin{array}{l}F= \\
p=\end{array}$ & $\begin{array}{r}2.40 \\
.04\end{array}$ & & $\begin{array}{l}=2.69 \\
=.02\end{array}$ & $\begin{array}{l}F \\
p\end{array}$ & $\begin{array}{l}=2.43 \\
=.02\end{array}$ & $\begin{array}{l}F= \\
p=\end{array}$ & $\begin{array}{l}2.82 \\
.01\end{array}$ \\
\hline
\end{tabular}


TABLE XIII

HYPOTHESES SUPPORTED AND NOT SUPPORTED BY THE STUDY

Hypothes is

Yes

No

I. Caregivers who live with the person with stroke will exhibit

* higher depressive symptomatology

* higher negative well-being

* lower positive well-being

than those live apart from the person with stroke.

II. Stroke severity, which includes assistance needed, negative personality/behavior characteristics, and communication impairment, will be significantly associated with caregiver well-being outcomes. Specifically, stroke severity at T4 will be positively associated with

* strain

* burden

* depressive symptomatology

* negative well-being

and negatively associated with positive well-being.

III. Caregiver stress (strain or burden) will be positively related to

* depressive symptomatology

* negative well-being

and negatively related to positive well-being.

IVa. Under conditions of high stress, caregivers who are

* satisfied with the amount of social contact they have with others

* satisfied with the quality of social contact

* have at least one reciprocal confiding relationship in their social networks

* perceive that they have informal support resources available if needed

$x$

$x$

$x$

will have significantly lower depressive symptoms

than other caregivers.

IVb. Under conditions of high stress, caregivers who have used coping strategies to deal with the

stroke will have significantly lower

* depressive symptoms

* negative well-being

and higher positive well-being than other subjects. 
Support was also found for the hypothesis which predicted buffering effects for satisfaction with the quality of social contacts, but not for the hypotheses which predicted buffering effects for the other social support variables or for coping strategies.

\section{CONCLUSION}

The conceptual model presented in Chapter III is well supported by the findings in this study. The block of variables included in the component of characteristics of the caregiver explained almost one third of the variance in depressive symptomatology at T4. The most important caregiver characteristics which were significantly associated with well-being outcomes were income, being an adult child of the person with stroke, satisfaction with the amount and quality of social support, and health status.

The characteristics of the caregiver which were significantly related to perceived stress were age, health status, and satisfaction with social support. Gender differences in relation to strain approached significance at $p=.07$, with females experiencing higher levels of overall strain than males in the caregiving role. A buffering effect was found for satisfaction with the quality of social support on depressive symptomatology in conditions of high strain and high perceived burden. Finally, caregiver age, health status, and satisfaction with social support were significantly related to the behavioral responses to stress.

The characteristics of the stroke which were significantly related to caregiver outcomes were changes in health status of the 
person with stroke and stroke severity. Although functional capacity of the person with stroke was significantly related to the psychosocial outcomes, a more comprehensive index of stroke severity (which included communication impairments and negative personality/behavior changes since the stroke as well as functional impairment) was significantly related to caregiver burden, strain, and adjustment to the stroke.

Both the index of caregiver strain and the caregiver burden scale were significantly associated with the psychosocial outcomes and with the behavioral responses to stress.

The following chapter addresses the final research question of changes over time, and presents the results from analyses of change in the outcome variables and in other selected variables over each point of data collection. 


\section{CHAPTER VII}

\section{CHANGES IN SELECTED VARIABLES BETWEEN T1, T2, T3 AND T4}

\section{INTRODUCTION}

The focus of this study is the investigation of long-term psychosocial outcomes for the caregivers of persons who have experienced a stroke. We have examined descriptive and statistical analyses of the T4 data in Chapters $V$ and VI. The next step in the analysis presented in this dissertation is the examination of changes over time in the psychosocial outcome variables and the experience of perceived caregiver burden. In addition, other selected items have been chosen for descriptive analysis in an attempt to explore several policy and practice implications of the data.

\section{CHANGES IN DEPRESSIVE SYMPTOMATOLOGY, POSITIVE AND NEGATIVE WELL-BEING AND PERCEIVED BURDEN OVER TIME}

Table XIV shows the means and standard deviations of the CES-D, POSWB, NEGWB, and perceived burden scores at each point of data collection. Although there was a decline of one point in CES-D scores between $T 1$ and T4, NEGWB increased very slightly. POSWB, which might have been expected to increase at least modestly after Tl only increased by two-tenths of a point by T4. Perceived burden, which was measured at $T 2, T 3$, and $T 4$ increased by more than one point between $T 2$ and $T 4$. 
TABLE XIV

MEANS OF CES-D, NEGWB, POSWB, AND BURDEN SCORES

FOR T4 SAMPLE OF CURRENT CAREGIVERS OVER TIME

$\begin{array}{llll}\text { TIME } 1 & \text { TIME } 2 & \text { TIME } 3 & \text { TIME } 4\end{array}$

\begin{tabular}{lrrrr}
\hline CES-D (60) & & & & \\
Mean & 12.1 & 12.5 & 11.2 & 11.1 \\
S.D. & 8.7 & 9.3 & 9.0 & 9.3 \\
NEGWB (15) & & & & \\
Mean & 3.7 & 3.5 & 3.9 & 3.9 \\
S.D. & 2.4 & 2.2 & 2.5 & 2.9 \\
POSWB (9) & & & & \\
Mean & 4.7 & 4.9 & 4.6 & 4.9 \\
S.D. & 2.4 & 2.5 & 2.2 & 2.6 \\
BURDEN (56) & & & & \\
Mean &.-- & 16.3 & 16.4 & 17.7 \\
S.D. & $-\cdots .8$ & 10.5 & 10.3 \\
\hline
\end{tabular}

In order to test for potential significant differences between these repeated measures, multiple analysis of variance (MANOVA) tests for repeated measures were performed to identify possible significant differences between each point in time of depressive symptoms, positive well-being, negative well-being or perceived caregiver burden. Given the small differences in the means at each point of data collection, it was not surprising that there were no overall significant differences in any of the repeated measures analyses.

However, since a score of sixteen on the 20-item CES-D is the generally accepted cut-off score for a diagnosis of clinical depression (Williams, 1985; Van Winkle, 1987; Price, 1987) it was also of interest to know whether there were changes in the percentage of the sample who scored at a level of 16 and above on the CES-D measure. 
Table XV illustrates the percentage of the sample who scored at 16 and above at all four points in time.

TABLE XV

PERCENT OF T4 SAMPLE WITH SYMPTOMS INDICATING CLINICAL DEPRESSION LEVELS ON THE CES-D MEASURE OVER TIME

TIME 1

TIME 2

TIME 3

TIME 4

Scores of 16 and above $33 \%$

$27 \%$

$29 \%$

$23 \%$

One third of the respondents in this study were at risk for a diagnosis of clinical depression at approximately the first two months after the onset of the stroke (T1). This percent is higher than is generally found in community samples of older persons but is lower than found in samples of chronically ill persons (Williams, 1985). The percentage of the sample at risk for clinical depression had decreased by six percent at $T 2$ (about six to eight months poststroke), but increased by two percent by the T3 one year follow-up. At T4, slightly less than one fourth of the sample (23\%) reported depressive symptoms which were at levels qualifying them for a diagnosis of clinical depression. Although there was an overall decline of ten percent between $\mathrm{T} 1$ and $\mathrm{T} 4$ in the prevalence of symptoms of clinical depression, almost twice as many caregivers in this study exhibit high depressive symptoms at $T 4$ than is the average rate of depression in community samples (12\%; Williams, 1985).

Further analyses of change in CES-D scores were conducted to identify the patterns among respondents who scored at levels of 16 and 
above over time. Ten percent of the caregivers at T4 qualified for a diagnosis of clinical depression at all four points in time. The largest percentage of "one time only" levels of clinical depression in the T4 sample of caregivers was at T2 (11\%). T1 and T3 each had six percent of the sample exhibiting high depressive symptomatology at just one point in time. Five percent of the sample reported clinical levels of depression for the first time at T4.

\section{OTHER ANALYSES OF CHANGE OVER TIME}

Other variables which have been identified in the literature as being particularly associated with caregiver burden, stress, or depressive symptoms have been chosen for descriptive analyses of changes over time. An exploration of these variables from a change perspective will take advantage of the longitudinal design of the study and will provide relevant information for understanding the policy and practice implications of the study. The variables chosen for descriptive analys is of change over time are:

* the proportion of the sample indicating changes in the specific personality/behavior characteristics of the person with stroke,

* the proportion of the sample indicating high scores on the six items of the perceived burden scale which are most significantly associated with depressive symptoms and caregiver adjustment,

* the proportion of the persons with stroke who are able to drive and provide their own transportation,

* participation in stroke clubs or caregiver support groups. 


\section{CHANGES IN PERSONALITY/BEHAVIOR CHARACTERISTICS} IN THE PERSON WITH STROKE

The measure of changes in stroke patient's personality and/or behavioral characteristics was introduced at $T 2$ and repeated at $T 3$ and T4. As seen in Chapter VI, Table VII, the six individual items on the personality/behavior characteristics scale which were significantly correlated with the outcome of depressive symptomatology at T4 were (1) angry, short-tempered (2) restless (3) jealous, possessive (4) forgetful, poor memory and (5) pleasant, easy to get along with. Twelve items were significantly correlated with the caregiver's adjustment to the stroke. These were (1) angry, short-tempered (2) self-centered (3) anxious, fearful, nervous (4) impulsive, uses poor judgment (5) suspicious (6) jealous, possessive (7) confused, perplexed (8) depressed, sad (9) thoughtful, considerate (10) laughs (11) pleasant, easy to get along with, and (12) happy, cheerful. Four additional items on the scale were significantly associated with perceived burden. These were (1) restless, (2) impulsive, (3) forgetfulness, poor memory, and (4) shows initiative, planning.

Table XVI shows the percent of the T4 caregivers who indicated that the person with stroke exhibited increases in negative personality/behavior characteristics or decreases in positive characteristics in the person with stroke at T2, T3 and T4. A11 of these characteristics were measured as changes since the stroke occurred rather than as changes since the previous interview. Although many behavioral characteristics are endorsed by similar numbers of respondents at each point in time, certain characteristics 
TABLE XVI

PERCENT OF T4 SAMPLE INDICATING NEGATIVE CHANGES IN PERSONALITY/BEHAVIOR CHARACTERISTICS SINCE THE STROKE IN THE PERSON WITH STROKE OVER TIME

\section{$\begin{array}{lll}\text { TIME } 2 & \text { TIME } 3 & \text { TIME } 4\end{array}$}

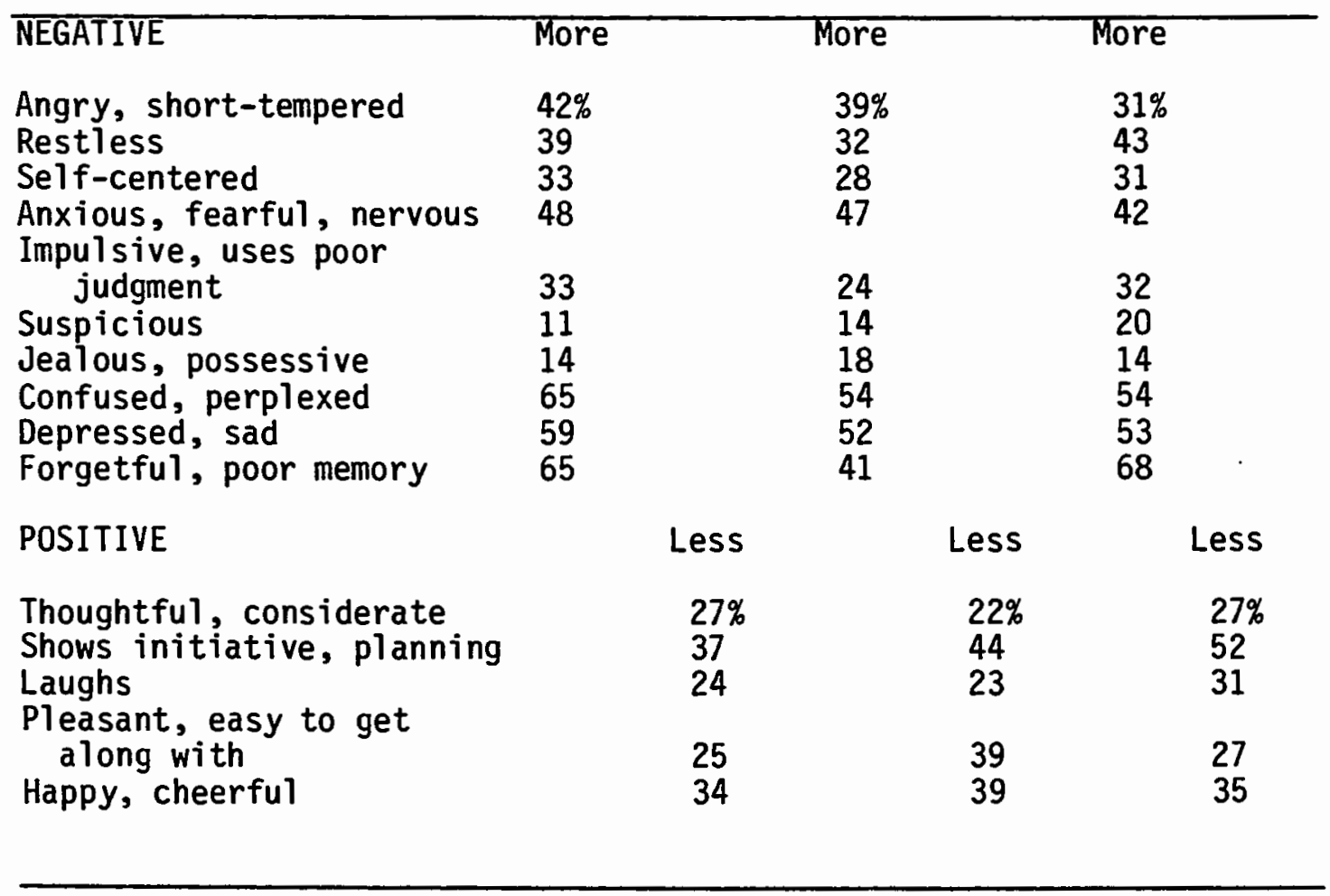

stand out as having gradually improved over time and others as having declined.

The most consistent improvement appears to be in the characteristics 'angry, short-tempered' and 'confused, perplexed', with fewer respondents indicating increases in these characteristics at each of the interviews. The improvement in 'confusion', with nine percent fewer respondents endorsing increases in this behavior, occurred between $\mathrm{T} 2$ and $\mathrm{T} 3$, and remained stable between $\mathrm{T} 3$ and $\mathrm{T} 4$. Six percent fewer of the respondents at T4 reported that the person 
with stroke showed increases in 'depressed, sad', or 'anxious, fearful, nervous' characteristics than had reported increases in those characteristics at at $\mathrm{T} 2$.

'Suspicious' was a negative characteristic significantly associated with caregiver adjustment and perceived burden. Between T2 and T4 the number of caregivers who reported increases in this behavior by the person with stroke almost doubled from eleven percent to twenty percent. Similarly, a decline in 'initiative, planning' in the persons with stroke also continued over time. Thirty-seven percent of the caregivers noted decreases in this characteristic at T2, but by T4 over half (52\%) of the caregivers reported declines in the initiative and planning capabilities of the persons with stroke.

Four characteristics seemed to be improving between $T 2$ and $T 3$ but had returned to T2 prevalence levels at T4. These were 'forgetful, poor memory', 'restless', 'impulsive, uses poor judgment' and 'thoughtful, considerate'. Forgetfulness in the person with stroke was endorsed by twenty-percent fewer caregivers at T3 than either T2 or T4, indicating that forgetfulness is not a condition which generally improves in a group of stroke patients over time. Similarly, increases in restlessness and impulsiveness on the part of the person with stroke were reported by seven to nine percent fewer caregivers at T3. However, more caregivers reported increases in those characteristics at T4. Although there were some increases at T3 in the numbers of caregivers who reported declines in the stroke patient's thoughtfulness, cheerfulness, and pleasantness, the 
percentage of respondents reporting declines in these characteristics remained relatively stable between $\mathrm{T} 2$ and $\mathrm{T} 4$.

\section{CHANGES IN SELECTED ITEMS OF PERCEIVED BURDEN}

The correlational relationships between the individual items on the perceived burden scale with CES-D and caregiver adjustment were presented in Chapter VI. Six items which were significantly correlated with both CES-D and adjustment at T4 have been chosen for further examination over time. These items have been selected because of their potential for being changed by caregiver intervention programs which are "psychoeducational" in design (Gallagher et al., 1986; Pratt et a1., 1987). They will presented through a descriptive analys is of the percent of respondents in the T4 sample who reported experiencing these items "often" or "almost always" in their role of caregiving for a person with stroke. As seen in Chapter VI, Table VIII, all of these items are correlated with both the CES-D scores and adjustment to the stroke at the $p<.01$ level. The six items are:

* "I feel the stroke patient makes requests over and above what he/she needs."

* "Because of my involvement with the stroke patient I don't have enough time to myself."

* "I feel stressed between trying to give to the stroke patient as well as to other responsibilities".

* "I feel the stroke patient currently affects my relationships with my family and friends in a negative way.

* "I feel that my social life has suffered because of my involvement with the stroke patient."

* "I feel that my health has suffered because of my involvement with the stroke patient." 
Table XVII shows the changes in these six items over the three points of time at which the data were gathered. Few changes occurred in the percent of T4 caregivers reporting that they 'often' or 'almost always' did not have enough time for themselves due to involvement with the stroke patient. Almost one fourth of the sample expressed a high degree of loss of personal time at each point of data collection.

TABLE XVII

PERCENT OF T4 SAMPLE INDICATING EXPERIENCE OF BURDEN

AS 'OFTEN' OR 'ALMOST ALWAYS' ON SELECTED ITEMS

OF THE CAREGIVER BURDEN SCALE OVER TIME

TIME 2 TIME 3 TIME 4

I feel the stroke patient makes requests of me that are above what he/she needs.

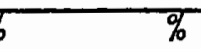

$\%$

Because of my involvement with the stroke patient I don't have enough time to myself.

I feel stressed trying to give to the stroke patient as well as to other responsibilities.

I feel the stroke patient currently affects my relationships with family and friends in negative ways.

I feel that my health has suffered because of my involvement with the stroke patient.

I feel that my social life has suffered because of my involvement with the stroke patient. 
In a similar fashion, there was little overall change in the percentage of respondents who felt that the person with stroke consistently made requests of the caregiver which were not really necessary. However, the percent of the sample endorsing this item increased by two percent between $\mathrm{T} 2$ and $\mathrm{T} 4$ whereas the percent of the sample who indicated consistent lack of personal time decreased by two percent between $\mathrm{T} 2$ and $\mathrm{T} 4$.

The consistent experience of stress between providing care for the person with stroke and other responsibilities decreased somewhat over time, with four percent fewer respondents indicating this condition at T4 than at T2. Among the six selected items from the perceived burden scale this is the only one which shows improvement between $T 3$ and $T 4$, with six percent fewer respondents indicating high levels of stress from competing responsibilities at T4 than they did at the one year post-stroke interview.

The number of caregivers who felt that the ir health had suffered because of their caregiving for the person with stroke doubled between T3 and T4 although it had declined somewhat between T2 and T3. At T4 ten percent of the entire sample felt that their health had been directly affected in a negative way by caregiving for the person with stroke.

The remaining two items from the perceived burden scale are the perceived effects which caregiving for the person with stroke has had on the caregiver's relationships with family and friends and on his/her social life. Both of these items were endorsed by increasing numbers of respondents at each point in time. At T4 over one fifth of 
the caregivers felt that their involvement with the person with stroke has hampered their social life. This figure represents a seven percent increase over T2. Fourteen percent of the caregivers feel that their involvement with the person with stroke currently affects their family and friendship relationships in negative ways at $\mathrm{T} 4$. This figure is almost double the amount of caregivers who regularly perceived negative effects of caregiving on their social life six to eight months after the stroke.

The examination of these selected items helps in understanding the long-term outcomes of caregiving on the primary caregivers of persons who have experienced a stroke. The results of these descriptive analyses indicate that as a whole caregiver stress and burden do not improve over time, nor do the stroke-related personality/behavior changes which have occurred for the person with stroke.

CHANGES IN THE MODE OF TRANSPORTATION FOR THE PERSON WITH STROKE

The continued ability to drive a car has often been anecdotally noted by older persons and their family members as a major sign of freedom and independence. The loss of ability to drive generally curtails a person's activity, social interaction, and sense of control over one's life. Since transportation has been acknowledged in the literature as a major task of caregivers, and a task which often creates problems for older caregivers (Unks, 1983), it was of interest to look at change in this variable over time. Providing transportation for the care-recipient is a primary task for caregivers, requiring 
large amounts of actual time as well as scheduling and planning. It is, however, one of the few ongoing tasks which is able to be shared by other family members as well as by friends and neighbors.

Table XVIII shows the stroke patient's ability to provide his/her own transportation by automobile at each time of data collection. Prior to the stroke (retroactively gathered at T1) over two thirds of the persons with stroke were able to drive and provide their own transportation. At T2 only $23 \%$ were able to do so. Because there were missing data for about 11 percent of the sample at T3 the percentages shown for the persons with stroke able to provide their own transportation (25\%) and the percentages of persons with stroke who are assisted in transportation needs by friends and relatives (50\%) are potentially under-represented by the T3 numbers on this table. There is an increase of at least two percent in the numbers of care-recipients able to provide their own transportation at T3. An additional two percent of the persons with stroke were able to drive at T4. However, fewer than half $(38 \%)$ of the persons with stroke who could drive before the stroke had recovered that capability by T4. Among those who indicated that the person with stroke was once again independently providing his own transportation were persons who had arranged for their car to be adapted with special controls or who had changed from driving a car to driving a motorized scooter. Over half $(57 \%)$ of the caregivers in this study were the major providers of transportation for the person with stroke at T4. 
TABLE XVIII

PERCENT OF T4 SAMPLE INDICATING STROKE PATIENTS' ABILITY

TO PROVIDE OWN TRANSPORTATION OVER TIME

Before Stroke TIME 2 TIME $3^{\mathrm{a}}$ TIME 4

Person with stroke able to provide own transportation

Friends and relatives (including caregiver) assist with transportation
$73 \%$

$16 \%$
$23 \%$

$25 \%$

$27 \%$

$a=$ about $11 \%$ of the T3 sample has missing data for this variable.

$b=$ because of a slight difference in the measurement of the transportation variable at T4 this figure represents the minimum percent of friends/relatives who provide assistance with transportation for the person with stroke at T4.

UTILIZATION OF STROKE CLUBS OR CAREGIVER SUPPORT GROUPS OVER TIME

The final variable of interest in the change over time analysis is the utilization of stroke clubs or caregiver support groups over the course of the study. As with transportation, there was a slight difference in the measurement of this variable between the original study and the T4 follow-up. However, comparisons are possible which may help in understanding the caregiver's attitudes and experiences with such programs.

Very few of the caregivers in this study attended stroke clubs or support groups. At T2 and T3 respondents were asked if they had attended any support groups or stroke clubs, and if those had been helpful. At T4 respondents were asked how often they had attended caregiver support groups or stroke clubs. The choices were sometimes, 
often or never. Ten percent of the T4 sample had attended a stroke club at least once by the time of the T2 interview. Of those, half indicated that the stroke club had been very heipful and the other half indicated that it had not been very helpfur. At T3 only $7 \%$ had attended a stroke club meeting since T2. About three-quarters of that small group had found the stroke club very helpful and the others had found it not helpful. Many of the respondents did not know if any stroke clubs were available in their geographical area. Others expressed no interest in any type of support group. Some of the respondents who had attended a stroke club meeting had found it to be very depressing. Most stroke clubs included the persons with stroke as well as the caregivers, and some of the caregivers in this sample found it not helpful to be with stroke patients who were "complaining all the time about how bad it is to have a stroke." Further correlational analysis of attendance at stroke club meetings indicated that attendance at a stroke club meeting was significantly correlated in a positive direction with perceived burden at both $\mathrm{T} 2$ and $\mathrm{T} 3(\underline{r}=.28$ $p<.01$ and $\underline{r}=.27 p<.01$ respectively). The respondent's mention of the degree of helpfulness of the stroke club was not significantly associated with either depressive symptoms or perceived burden at 12 or T3.

Twelve percent of the sample had participated in some type of caregiver support group by T4. Of those, about three fourths (71\%) attended "often" and the others attended "sometimes". An additional $4 \%$ of the sample had attended some type of caregiver training program. The caregiver training programs were described as being part of the 
immediate post-stroke therapy for the person with stroke, with the caregiver training specifically focused on how to assist the stroke patient with tasks such as bathing, dressing and exercise. Even at T4 attendance at caregiver support groups and caregiver training courses were both significantly correlated in a positive direction with depressive symptomatology ( $\underline{r}=.22, p<.05$ and $\underline{r}=.22 p<.05$ respectively).

\section{CONCLUSION}

This analysis of change over time in selected aspects of the caregiver's experience supports findings from other studies that caregiving is a role which has long-term negative aspects. Time itself does not necessarily make things better.

Especially interesting are the changes over time in the depression scores. We have seen a relatively consistent mean score at all four points in time, yet ten percent fewer caregivers reported levels qualifying them for diagnosis of clinical depression at T4 than at T1. Because there is a prognosis for recovery from stroke, and there was evidence of improved health in the persons with stroke in this sample between $T 3$ and $T 4$, it is reasonable to expect that some of the caregivers will report lower depressive symptoms over time. However, the reduction in numbers of respondents who report high levels of depression, accompanied by a stable average score over time, indicates that some of the respondents are becoming increasingly more depressed over time. In addition, the consistent finding of significant relationships between attendance at stroke clubs or caregiver support groups with depressive symptoms and/or perceived 
burden at T2, T3 and T4 indicates the critical need to explore these relationships and more closely examine their association with caregiver well-being.

The loss of ability to drive, with its associated dependence on the primary caregiver and other persons, also appears to be relatively consistent over time. The resulting long-term dependence on others could be related to the negative behaviors which in turn are associated with negative outcomes for the caregiver.

Further discussion of the findings of this study, with recommendations for policy and practice considerations will be presented in Chapter VIII. 


\section{CHAPTER VIII}

\section{DISCUSSION AND IMPLICATIONS}

\section{INTRODUCTION}

This study provides significant information about the longterm experience of caregivers of persons with stroke, and adds to the body of literature now available. In addition, the findings have generated some directions for future research and have identified areas to be addressed in the design and planning of caregiver programs.

This chapter is divided into four sections: 1) discussion of the findings in relation to the research questions which were raised and the hypotheses which were postulated in Chapter III, 2) discussion of the limitations of the study and cautions in interpreting the findings, 3) suggestions for further research and 4) presentation of the policy and practice implications of the study.

\section{DISCUSSION OF THE FINDINGS IN RELATION TO THE RESEARCH QUESTIONS}

The first research question queried about the differences which might occur in the outcome measures of depressive symptomatology and psychological well-being between those respondents who were still in the support role for the person with stroke at T4 and those for whom the person with stroke had died. Some of the literature has 
indicated that the caregivers who are best off at follow-up interviews are those for whom the care-recipient is no longer living (Borgatta and Montgomery, 1987). Unfortunately, the findings of this study provide inconclusive evidence about this issue. Although there were no significant differences on any of the three outcome measures between caregivers who were stil1 the primary support person and those for whom the person with stroke had died, there was a significant correlation between length of time since the death of the person with stroke and the measure of depressive symptoms. The respondents who had experienced the death of the person with stroke within several months of the T4 interview could be expected to be experiencing grief and its accompanying depressive symptoms. Therefore, the finding of no difference between current and former caregivers in this study is interpreted cautiously as being due to the confounding of the grief process among the more recently bereaved respondents. It is possible that over time they would be significantly less depressed than the respondents who remain in a caregiving role.

The second area of interest was in the tasks and assistance which caregivers actually provide for the person with stroke, and what type of assistance they themselves receive in providing this help. The findings of this study support previous findings that the caregiver role falls predominantly to one family member (Horowitz, 1985; Gibson, 1984; Stone, 1987) and that little regular assistance is provided by other helpers. Personal care tasks (bathing and dressing), supervision, and telephone check-up were provided by approximately twenty-percent of the sample. Assistance with bill- 
paying and correspondence, transportation, shopping, household chores, meal preparation, and regular visiting are provided by nearly half of the respondents. Most of the caregiver tasks were generated by the onset of the stroke. Caregivers who were wives were most likely to have been doing regular meal preparation, bill-paying, and household chores before the stroke. Fewer than half of the respondents who report that they prepare meals for the person with stroke were doing that task prior to the stroke onset. None of the caregivers had provided bathing, dressing, help with ambulation, or telephone checkup prior to the stroke.

This study shows that transportation is a significant long-term need for persons who have had a stroke. Only thirty-eight percent of the persons with stroke who were able to drive prior to the stroke had recovered their ability to drive a car several years later. For stroke patients who live alone, and for the older couples whose only driver was the person with stroke, the loss of independence which driving provided could be expected to have negative impacts on both the person with stroke and the caregiver. Almost one-fifth of the respondents in Unks' (1983) study of elderly wives of stroke patients reported that transportation was the most troublesome task they had to face as caregivers. This finding has implications for community-based transportation policy and planning.

Respite care is an area which relates to the tasks and responsibility of caregiving, even though it is a support service for the caregiver instead of something done by the caregiver for the person with stroke. Although thirty-five percent of the caregivers 
indicated that the person with stroke was unable to be left unattended, relatively few had respite time provided by family members at least several times a month. None of the respondents used the respite services of formal agencies. Fifty-two percent of the caregivers "sometimes" took time just for themselves (though not necessarily away from the home) and twelve percent said they "never" were able to take time for themselves. One reason given for not having someone come in to the home for caregiver respite was that the person with stroke objected to having other people come to stay with him/her. Other reasons were lack of financial resources to pay for someone to come, and that family and friends "have their own lives."

Unlike the findings of some other studies (e.g., Zarit et al., 1986: Cicirelli, 1983), the results from this study showed significant correlations between the amount and frequency of caregiver tasks and both perceived burden and depressive symptomatology. In addition, the amount of help provided for the person with stroke by the caregiver was significantly associated with the severity of the stroke. These relationships suggest that negative caregiver outcomes for this sample are grounded in objective caregiver responsibilities rather than (or as much as) in predominantly subjective responses to the caregiving role. One might conclude that these findings would support a recommendation for affordable and easily accessible community-based home health and transportation services as ways to minimize the negative effects of providing care for a functionally disabled family member. However, the results of the study also show that few such 
services are actually used by the respondents, perhaps because of the high cost of such services if they are used over a long period of time.

A related finding is that financial strain was not very high for this sample of caregivers. Although this finding is similar to that of other studies (e.g., Brody, 1985; Stephens and Christianson, 1986) and at first glance is interpreted as good news, it is very possible that financial strain is low because most caregivers do not purchase support services. It is important to note that stroke is an acute illness for which treatment, rehabilitation, and home health services are covered by Medicare and other medical insurance policies within the first six months or so of the stroke onset (Kaufman and Becker, 1986). Many of the respondents in this study learned their strokerelated caregiving skills during this period of time in order to provide those services on their own when the professional services were no longer covered by insurance. If caregivers were to purchase enough professional services to lower the levels of strain, burden, and depression which are related to the caregiving role, the reported levels of financial strain would probably be very much higher.

The third area of query in this study concerned identifying the correlates and predictors of the outcomes of depressive symptomatology, positive well-being, and negative well-being. As we have seen in Chapter VI, the characteristics of the caregiver, especially as they relate to subjective health, the presence of social support and a sense of satisfaction with their support systems have more weight in predicting caregiver outcomes than do the 
characteristics of the person with stroke. The lack of significant gender differences in strain or burden for this sample may be due to the passage of time, as has been noted by Zarit et al. (1986). Females in this study reported higher levels of strain than males at a significance level of $p=.07$ which is indicative of slightly higher levels than might be attributed to chance. Zarit et al. (1986) found that gender differences in strain and burden had disappeared at a twoyear follow-up interview. The authors postulated that husbands were able to use instrumental task-focused approaches to the caregiving role sooner than wives, who needed to deal with the emotional aspects of losing a spouse to Alzheimer's disease before they could assume an instrumental approach to providing care.

Although the characteristics of the caregiver contribute more to negative long-term outcomes than characteristics of the stroke, it is important to note that the severity of the stroke as characterized by need for assistance, negative personality/behavior characteristics, and communication impaiments was significantly related to caregiver strain, perceived burden, depressive symptomatology, and negative well-being. These significant relationships are in contrast to Wade et al. (1986) who found that none of the stroke-related variables were related to caregiver depression two years after the stroke. However, Wade et al. (1986) measured only functional capacity of the person with stroke rather than including other neurological indicators of stroke severity. The importance of including the neuro-psychological effects of stroke and communication impaiments in measures of longterm stroke severity is one of the important findings of this study. 
Pratt et al. (1987) found that the individual items of burden which had the highest mean scores for caregivers of both communitydwelling and institutionalized elders were those which assessed the perception that the relative was dependent on the caregiver, the perception that the elder feels that only the caregiver can provide care, fear of what the future holds for the relative, and stress between caregiving and other responsibilities. On the other hand, compared to caregivers for institutionalized patients, caregivers for community-dwelling elders gave significantly higher ratings to items that assessed lack of time for self, negative impact of caregiving on their social life and on relationships with friends, and lack of privacy. The findings of this dissertation study support those of Pratt et al. (1987) that having time for oneself and time to nurture one's own personal relationships is very important to the long-term psychological well-being of family caregivers.

Some of the aspects of burden which are highly correlated with negative outcomes such as not having time for oneself or one's own social life because of involvement with the person with stroke may be readily amenable to change through education and training in behavior modification and coping techniques (Gallagher et al., 1987). The learning of these skills would be useful at any time past the onset of stroke, as evidenced by Unks' (1983) finding that the respondents in his study expressed greater need for psychological information, support, and a rest from caregiving after the first year than they felt they needed early after the stroke onset. 
The caregiver's adjustment to the stroke is an important factor in both depressive symptomatology and positive well-being at T4. The caregiver's perception that he/she has adjusted "very well" to the stroke is associated with lower depressive symptoms and higher positive well-being. The question about adjustment to the stroke was stated as, "Overall do you feel that you have adjusted to the stroke?" with categories of "no/not quite " to "very well". Verbatim responses were recorded to the follow-up question "what are some of the things that have made adjustment difficult?" or "what has helped you to adjust?" Content analysis of these responses did not elicit any pattern to either adjustment or lack of adjustment. One respondent responded, "How do you adjust? I'm surviving." Another said he adjusted because of "My make-up. I'm able to cope with things." A third said, "I've adjusted because there's nobody to do it but me. You have to adjust to your confinement. You have to rest. You have to adjust to the things you need to do because you can't afford help." A sister of the person with stroke said "I love her. If you love someone you overlook a lot". And a spouse, whose husband had had the stroke almost four years prior to the T4 interview said, "I just can't accept it. I feel as though I should have better control of my emotions. I can't accept seeing him as he is. The (day-care center) people have helped me to get him to do more for himself. But it's hard to watch him struggle." Another respondent attributed her adjustment to the fact that the stroke patient was "determined to be independent and didn't give in to the stroke". 
Several respondents attributed their adjustment to having had a career in nursing or having had experience with stroke because another family member had survived a stroke. Some other respondents mentioned that the speech therapist had been helpful in their adjustment to the stroke. Speech therapy was the one component of rehabilitation programs that lasted beyond the first six months after the stroke. Some of the caregivers at T4 mentioned that speech therapy was still being continued for the person with stroke. The long-term relationship of the speech therapist with both the stroke patient and the caregiver, plus the consistent encouragement and help with communication which the speech therapist provides for both persons may be key elements in adjustment for caregivers of stroke patients who have serious speech impairments.

An important recommendation from this study for further examination is that the construct of adjustment to stroke by the caregiver be more clearly defined and tested. Adjustment may be understood in two distinctly different ways which are not clearly defined in a general question about adjustment. For example, adjustment may be understood as an acceptance of the limitations of a situation or of impairments. In contrast it may also be understood quite differently as the ability to actively seek resources to overcome limitations or impairments. "Lack of adjustment" may serve as a motivator for seeking assistance and support. The development of a reliable and valid scale which would adequately tap the construct of adjustment would be a useful contribution to the planning of caregiver education and intervention programs. 
A fourth set of research questions addressed by the study asked, "Do the primary support persons see themselves as 'caregivers'?" If so, what effect does this perception have on the outcomes of depressive symptomatology or psychological well-being? What relationship does it have to the experience of strain, burden, and adjustment to the stroke?

Sixty-two percent of the respondents in the sample perceived themselves to be caregivers. This may be partially due to increased awareness of their support role during the course of the study's four interviews, and to the increased visibility of caregiver programs in the community. However, almost 40 percent of the same sample did not view themselves as caregivers, so it is hard to ascertain the effect of having been in the study on the responses to this question.

The caregiver's perception that he/she was a caregiver for the person with stroke was significantly associated with depressive symptomatology but not with negative or positive well-being. Perception of self as a 'caregiver' was also significantly associated with higher levels of perceived stroke severity, so that respondents who saw themselves as 'caregivers' were likely to be providing more support for the person with stroke than those who did not. Although only twelve percent of the sample indicated that they had attended caregiver support groups or training courses, these coping strategies were also highly correlated (not surprisingly) with perception of self as caregiver. It is puzzling that attendance at stroke clubs or caregiver support and training sessions was significantly correlated in a positive direction with depressive symptomatology and perceived 
burden at all points of data collection after the initial interview. Perhaps caregivers who are depressed and burdened are those who are most likely to seek support and encouragement from caregiver programs. If so, this finding has several implications for caregiver interventions and educational programs. An evaluation component, using valid and reliable scales.for the outcome measures, should be included in every type of program. Data about effectiveness in reducing burden, depressive symptoms, and other negative outcomes for the caregiver are crucial for developing an adequate array of useful programs. In addition, "Caregiver Effectiveness" or "Coping with Caregiving" classes could provide beneficial skills as well as support. Such courses have been developed by Gallagher et al. (in press) following the model of "Coping with Depression" classes which were originally developed by Lewinsohn, Antonuccio, Steinmetz and Teri (1986). Gallagher et al. (in press) report significant decreases in level of depression and increases in morale for respondents in the psychoeducational program as compared to a waiting list condition (some of whom were also members of a caregiver support group). Kahan et al. (1985) report similar decreases in burden in family caregivers of Alzheimer's patients who have attend such a "Coping with" course.

The second issue raised by the finding that perception of oneself as a caregiver is associated with negative mental health outcomes relates to policy. Should families be expected to provide care for family members who are in need of long-term support services for chronic disabling conditions even if there are high emotional or physical costs involved in providing that care? The emphasis on the 
family as a major provider of long-term care for the disabled and frail elderly is high, and most caregiver studies report with a certain degree of pride the findings that families have not abandoned their older relatives but in fact are caring for them more than ever before (Shanas, 1979a; 1979b; Cantor, 1983; Brody, 1985; Stone, 1987). However, Borgatta and Montgomery (1987) postulate that caregiving by the informal system may be due to the lack of other options rather than to personal choice. Spouses have the least choice in alternatives for care because the elderly do not have the right to public assistance unless they are in financial need. A spouse, therefore, must share in the poverty necessary to obtain publicly-funded services. Adult children, on the other hand, may pay for assistance, let the parent pay for assistance, perform the helping tasks, or allow the parent's income to diminish to the point of eligibility for services. They have more choices than spouses, and they do not have to share in the poverty necessary to obtain services if spending-down the income is the only alternative. Borgatta and Montgomery (1987) suggest that the evidence that adult children who are primary caregivers tend to seek and use more formal services than do spouses reflects their greater choices and the ability to use the parent's resources to pay for services at no cost to themselves. Spousal caregivers, on the other hand, must consider the expense of services in relation to the financial resources of the marital dyad. Borgatta and Montgomery (1987) indicate that the additional evidence that middle-class children tend to provide more financial assistance and working-class children provide more direct service presents another 
reason to believe that when alternatives are available direct caregiving is not the option that is most often chosen. Their ultimate conclusion, which is discussed in more detail later in this chapter, is that families assume the role of the caregiver as a consequence of values imposed by public policy rather than from their own personal values.

\section{DISCUSSION OF FINDINGS IN RELATION TO THE HYPOTHESES}

We have seen in Chapter VI that the first hypothesis, that caregivers who live with the person with stroke will exhibit more depressive symptoms, higher negative well-being, and lower positive well-being than those who do not was not supported by the findings. The lack of support for this hypothesis may have been due to several factors. The first is that the distribution of caregivers living with versus apart from the person with stroke was not balanced between spouses, adult children, and other caregivers. Whereas all of the spousal caregivers were living with the person with stroke, eight percent of the non-spousal caregivers were caring for stroke patients who were placed in long-term care facilities. Studies which have compared the well-being of caregivers who have placed a relative in a long-term care facility and those who have not indicate that negative outcomes remain high for the caregiver even after placement (Pratt et al., 1987; Zarit et al., 1986). In addition, Cicirelli's (1981) construct of 'filial anxiety' which states that adult children become anxious, concerned, and protective when a parent becomes dependent, 
may be operating to increase the levels of negative outcomes for adult children who live apart from the parent who has suffered a stroke.

A third possible factor in the finding of no difference between the two groups is that several of the spousal caregivers who had been caring for a severely impaired person with stroke at home did not participate at T4 either because the stroke patient had died within the past month or because they were too heavily involved in the care. One wife was not able to participate when called because she was taking her husband to the hospital and asked to be called back in a few weeks. She was heavily stressed again when called the second time, and requested a call back after the Christmas holidays. When contacted in January, she said that her husband had died a few days after Christmas. Another caregiver who was caring for a severely disabled husband had a stroke herself between the telephone interview and the time she was able to complete and return the self-report packet. The data from her entire interview were omitted from the study because the missing self-report packet included outcome measures as well as the measures of perceived burden, the Barthel Index, and the measure of stroke-related personality/behavior changes. Therefore, the inability of the heavily stressed spouses who were living with severely impaired stroke patients to participate at T4 may have also contributed to the finding of no difference between the caregivers who lived with versus apart from the person with stroke at T4.

The second set of hypotheses, which postulated that an index of stroke severity comprised of behavioral changes, assistance needed, 
and communication impaiments would be significantly related to the well-being outcomes, and that high levels of stroke severity would result in high stress, high depressive symptomatology, high negative well-being and low positive well-being were all supported by the findings. The extent of stroke severity is not necessarily measured only by functional capability of being able to adequately manage the activities of daily living (ADLs). The finding that caregiver adjustment to the stroke is significantly related to the measure of perceived stroke severity but not to the Barthel Index measure of functional capacity provides support for the suggestion that a comprehensive measure of long-term stroke severity should include residual negative personality/behavior characteristics and communication impaiments as well as physical disability (Tompkins et al., 1988).

The third hypothesis proposed that stroke-related caregiver stress would have main effects on caregiver depression, negative wellbeing, and positive well-being. This hypothesis was supported by the findings of this study. Both strain. and perceived burden were significantly correlated with the outcomes, and perceived burden itself was a significant predictor of negative well-being at T4.

It is clear from several studies that the relationship between the severity of the care-recipient's impairments and the experience of burden by the caregiver is very complex (Zarit et al., 1986; Morycz, 1985; Pratt et al., 1987). For the caregivers of persons who have had a stroke, the complexity of this relationship may well relate to the negative personality/behavior changes discussed above. Zarit et al. 
(1986) suggest that although the patient's impairment may have been the initial cause of perceived stress, the intensity of the burden which was felt by the caregiver depended on how well the caregiver coped with specific problems and what resources were available, especially for assistance with tasks and occasional relief from caregiving.

The final set of hypotheses postulated that certain social support variables and coping variables would have buffering effects between stress and both depressive symptoms and negative wel1-being. The only significant buffering effect was found for satisfaction with the quality of the caregiver's social contacts. Caregivers who were very satisfied with the quality of their social contacts had significantly fewer depressive symptoms in conditions of high strain and high perceived burden than those who were less satisfied with the quality of their social support system. No hypothesized buffering effects were found for either satisfaction with amount of social contact, presence of reciprocal confiding relationships, perceived availability of social support, or coping strategies.

The importance of satisfaction with the quality of social support as a significant buffer which lessens caregiver depression in situations of high stress and burden points to the need to develop intervention programs or information dissemination which increases awareness of family members and friends of caregiver stress and concerns. New educational programs are now being offered by hospitals to teach community volunteers the skills for making supportive and helpful hospital visits; perhaps similar community-based programs 
could be developed for people whose close friends or family members have primary caregiving responsibilities for an impaired adult. Pratt et al. (1987) found that family caregivers do not always know how to mobilize their support systems for their best advantage. Therefore, they too suggest developing programs which would involve the support persons of the primary caregiver or would teach the caregiver skills to use in mobilizing support for themselves in their caregiving tasks.

The study presented in this dissertation has tested unidirectional relationships between the variables in the model. Because the variables measuring adjustment, coping, and perception of self as caregiver were added at $T 4$, a potential aspect of the model not tested in this study are the effects over time of the interaction between these responses to the caregiving role and strain, burden, and even the personality/behavior characteristics of the person with stroke. An important next step in stroke patient/caregiver research would be to test the effect of caregiver behaviors on the stroke severity and stress measures. It is likely that a feed-back loop could exist, especially if the caregiver were to learn specific communication or coping skills which might either improve the stroke patient's behaviors or the experience of stress and burden for the caregiver. A recent study which has investigated the effects of stroke on both the person with stroke and the caregiver (Stephens et a1., 1987) has found that stroke patients' experience of negative interactions with their caregivers is a significant factor in lower morale and greater psychiatric symptoms in the stroke patient. These negative interactions were most likely to be identified in the area of unwanted 
assistance with basic ADLs (specifically dressing and grooming). The interactions between the stroke patient and the caregiver over time could be tested by this model and provide even more specific data to enhance our understanding of the long-term impacts of stroke on both persons.

\section{LIMITATIONS OF THIS STUDY}

The fact that the sample was not generated by random selection, and that respondents of lower socio-economic status did not remain in the study over time, limit the generalizability of the findings to other populations. However, since the findings provide evidence of the relationship of lower income with higher levels of burden and depressive symptoms, it is reasonable to think that respondents of lower socio-economic status will have even greater negative impacts from providing care for a person with stroke than have the respondents in this study. Since many caregiver intervention and education programs are hospital-based it is recommended that special efforts be made by hospital discharge planners to connect persons of lower socioeconomic status with the caregiver outreach programs.

One of the strengths of this study is the method of sample selection. Unlike some studies of the effects of caregiving which have drawn samples from caregiver support groups or from selfidentification of caregivers (e.g., Pratt et al., 1987; Zarit et a1., 1986; Stone, 1987), the caregivers were named by the person with stroke him/herself as being a primary support person. This selection process may tend to offset the non-random selection process and enable 
us to interpret the findings as generalizable to populations of persons who are providing care and support for a functionally disabled person but do not particularly identify themselves as "caregivers".

\section{AREAS FOR FUTURE RESEARCH}

The findings of this study suggest a need for future research in several areas. The first area relates to bio-medical research on stroke itself. Basic research on the causes and prevention of stroke must continue to have high priority in medical research. Mortality rates from stroke have declined largely through treatment of hypertension and the technological capability for preventive surgical procedures (Ratcheson and Selman, 1987) but morbidity rates and disability remain high. The long-term disabilities which a stroke leaves in its wake have been clearly seen in this and other studies (Silliman et al., 1988; Stephens et al., 1987; Jones and Vetter, 1984). Persons with stroke often require long-term periods of nurture, support and caregiving which may seriously and negatively affect the caregivers. Twenty-eight percent of the stroke patients in this study had at least one additional stroke between $T 1$ and T4. Thirty percent of the respondents said that worry about another stroke was their major stroke-related concern at T4. As several explained, their real fear was that the next stroke would be "even more devastating", and that neither the family nor the person with stroke would be able to cope any longer.

A second stroke-related need for research lies in extending the research on rehabilitation for stroke patients. Especially 
important is research to test the value of intermittent rehabilitation geared to teaching and practicing self-management skills, adaptation skills, behavior modification, and socialization. Such a program was developed by Pacific University in Forest Grove, Oregon (Tangeman, Banaitis, Williams \& Wingfield, 1987) for stroke patients who had experienced their strokes at least one year before entry into the program. Analysis of the results showed significant improvements in activities of daily living functions and in interest and pleasure in socialization. One regular activity of the 5-week intensive program at the Stroke Management $\mathrm{Clinic}$ was having lunch at a restaurant. Quite a few of the stroke patient participants had not done so since the stroke, and they were able to overcome their feelings of uneasiness and embarrassment in a social setting. This finding relates to Hyman's (1971) concept of stroke patient's residual sense of "stigma" even when severe functional disability is not apparent.

Although the Stroke Management Clinic did not include a program for caregivers, at least two of the respondents in the T4 study mentioned that their spouses had attended the Pacific University's Stroke Management $\mathrm{Cl}$ inic five-week program. These respondents said "it was the best thing that happened for both him and for me." The fact that these respondents experienced beneficial effects from the stroke patient's participation suggests that future research should examine the bi-directional effects which occur with caregiver or stroke patient interventions over time.

In addition, the suggestion made by Tompkins et al. (1988) that called for extension and further validity and reliability testing of 
the personality/behavior characteristics scale which was used throughout this study is supported by the T4 findings. This scale, which was developed by Rau (1986) for use in this study at T2, has been an important indicator of some of the lasting effects of stroke which are likely to have significant long-term impacts on the mental health of the caregiver.

The second set of areas identified for research by the findings from this study relate to the planning and implementation of caregiver programs. It has been suggested earlier that an evaluation component be part of every caregiver intervention program. The evaluation should be designed as a pre-test/post-test study. Although the ideal would be to have a control group, it might not be possible for both ethical reasons (withholding helpful information from a caregiver in distress would not be acceptable research procedure), economic reasons, and the lack of training by program staff to carry out a relatively sophisticated evaluation. However, it seems possible that a valid and reliable program evaluation instrument could be developed by experienced caregiver researchers and used nationally by a variety of caregiver programs to test the effectiveness of programs in decreasing caregiver distress. Such an evaluation instrument would be most helpful if it were geared to measuring outcomes in relation to different caregiver groups such as caregivers of the neurologically impaired, caregivers of persons with Alzheimer's disease, or caregivers of frail elderly. The heterogeneity of caregiver measurements and programs is an area which has been recognized as a 
problem (Gallagher et al., in press) but has not yet been developed in the literature.

Another recommendation for further research based on the findings from this study is that longitudinal studies be designed which tap the recursive nature of caregiver/care-recipient behaviors and outcomes. Such studies might be very much like a replication of the first three waves of this study but with caregiver adjustment, coping, and perception of self as caregiver included from the beginning.

In sum, it is important to recognize that burden and general distress may stay high or even become worse over a period of several years for the primary caregivers of persons who have had one or more strokes. That finding was also supported by Unks (1983) who noted that the wives who had been caregivers for their stroke victim husbands the longest (four to five years) had the lowest morale scores in the entire sample. Longitudinal research which would tap the dynamic effects of the interactions between the caregiver and the person with stroke, perhaps in the context of an intervention program, would be beneficial for further understanding of how to improve the long-term outcomes for caregivers of persons with stroke and other neurological impairments.

\section{IMPLICATIONS OF THE STUDY}

As mentioned in an earlier section of this chapter, several major issues emerge from the findings in this study. The most major one concerns the need for a national health policy which acknowledges 
the need for affordable and accessible services to caregivers for persons of all ages whose capacity for self-care is restricted. Although efforts are being made to consider ways to fund the provision of such services in the community, the approach to such considerations seems to be more from a crisis-oriented incremental position rather than from a clear set of goals and purposes. Increased longevity, combined with increased numbers of persons who have survived but are chronically disabled by strokes, or heart disease, has created a new population of persons in need of community-based support services (Hughes, 1985). Decision-makers in the policy arena cannot simply look at ways to save money on the care of severely impaired persons who need constant nursing care. They must begin to consider ways to meet the needs of this new population. There must be specific goals and objectives which address the issues of quality of life for the chronically disabled and their caregivers, and which have a purpose of providing equitable access to affordable and comprehensive services.

Borgatta and Montgomery (1987) suggest that most policy analysis in this area has been to conceptualize the problem as one of achieving a balance of responsibility between the family and the state. They argue, however, that policy analysts should look beyond the existing structures and the personal value systems on which current policies are based. Instead of the current basis of assuming that the family should be the primary provider of chronic care, they suggest that policy should be based on the question "How does the state assume responsibility for the care of elderly persons who are unable to care for themselves?" and not the question of how the state can "support" 
the family in its caregiving role without spending too much money or without substituting paid services for those which had previously been supplied for free.

Several studies which have compared health care policy in several different countries (Gibson, 1984; USGPO, 1984; Kane and Kane, 1985) note that the expansion of programs to meet new needs is directly associated with the wealth and financial resources of the country. Kane and Kane (1985) suggest that the United States could benefit from the experience of Canada, in which several of the largest provinces have made nursing home and home care benefits available without means-testing and with relatively little consumer costsharing. Kane and Kane (1985) argue that if strategies such as case management are developed to allocate resources, encourage appropriate quality, and control costs in programs with high natural demand, universal access to services for persons with chronic disabilities may not only be cost effective but may actually result in holding down the costs of such programs.

A major policy concern about providing services to the elderly which families have already been providing has been that provision of supportive services by the formal system would replace the family as the provider of care for the functionally disabled. However, studies have shown that this has not happened, and a general conclusion of the literature is that formal agency support appears to be an extension of, rather than a substitute for, services provided by the family. Formal help frequently appears to fill service gaps, rather than 
substitute for services, especially in cases where older persons require intensive and frequent assistance (Gibson, 1984).

Expansion of in-home services has been seen by some researchers to enhance and strengthen the capabilities of families to care for their older members as opposed to undermining family support (Gibson, 1984; Stone, 1987; Stephens and Christianson, 1987). However, expansion of in-home services, and the development of guidelines which allow access to those services at reasonable cost, has its own important policy implication which cannot be ignored. People who are caring and qualified must be willing to provide those services and fill the jobs created by increased demand. Oregon, a state which has supported in-home and community-based services for Medicaid clients since 1981, is now experiencing a lack of workers to fill the homeservice jobs in the health-care arena (Nonnenkamp, personal communication). Homemaker and home health aides who work for agencies such as Visiting Nurses' Association are paid lon hourly wages and are not provided with healthcare insurance or other employment benefits. Concomitant with developing a public policy which increases demand for these services will be a policy to provide screening, training, and adequate wages for the people who will be needed to provide them.

The second major issue which arises from the findings in this study regards caregiver intervention programs. It is clear that inclusion of the caregiver in the treatment plan of a chronically ill or disabled person is important. In addition, it seems that models of caregiver intervention and education programs could be developed which would be tested for their results in decreasing depression, burden, 
and negative well-being. Unless public policy is developed which removes the primary responsibility of caregiving from the family, caregivers are likely to to be involved in long-term support of more than one elderly relative. Interventions which could teach "stress management" skills or "coping" skills might well serve to protect the caregiver from being drained by the initial caregiving experience.

\section{SUMMARY AND CONCLUSION}

Throughout this study on the caregivers of persons who have experienced a stroke we have seen that in general the experience of caregiving is a stressful one with some long-term negative impacts on the caregiver. The fact that family members do supply personal care services to functionally impaired relatives for very long periods of time is a generally new phenomenon in our society. The consequences of a public policy which does not provide access to supportive services may prove counter-productive over the next few decades as families' resources to continue caregiving are diminished. Recommendations which arise from this study include 1) the development of a national policy which provides for direct access to affordable community-based personal care and support services to persons whose capability for self-care is restricted, and 2) the development of programs for family members and their own support persons which will teach effective skills in coping, communication, and stress-management. 


\section{REFERENCES}

Ahlsio, R., Britton, M., Murray, V., Theorel1, T. (1984). Disablement and quality of life after stroke. Stroke, 15, 886-890.

Antonucci, T. C. (1985). Personal characteristics, social support, and social behavior. In R. H. Binstock \& E. Shanas (Eds.) Handbook of aging and the social sciences. Second edition. New York: Van Nostrand Reinhold.

Arling G., \& McAuley, W.J. (1983). The feasibility of public payments for family caregiving. The Gerontologist, $23(3), 300-306$.

Artes,R. and Hoops, R. (1976). Problems of aphasic and non-aphasic stroke patients as identified and evaluated by patients' wives. In Y. Lebrun and R. Hoops (Eds.), Zeitlinger B.V.

Baum, H. M. (1982). Stroke prevalence: An analysis of data from the 1977 National Health Interview Survey. Public Health Reports, 97, 24-30.

Baum, H. M., \& Robins,M. (1981). The National Survey of Stroke: Survival and prevalence. Stroke, 12 (Part 2, Suppl.1), I-59-I68.

Belcher, S. A., Clowers, M. R., \& Cabanayan, A. C. (1978). Independent living rehabilitation needs of post-discharge stroke persons: A pilot study. Archives of Physical Medicine and Rehabilitation, 59, 404-409.

Benedict, A., Lovett, S., Gallagher, D. (1986). Morale and burden in adult child and spousal caregivers. Paper presented at the 39th Annual Convention of the Gerontological Society of America, Chicago.

Berkman, P. (1971). Measurement of mental health in a general population survey. American Journal of Epidemiology, 94, 105111.

Berman, S., Delaney, N., Gallagher, D., Atkins, P., Graeber, M. P. (1987). Respite care: A partnership between a veterans administration nursing home and families to care for frail elders at home. The Gerontologist, 27(5), 581-584.

Berman, W. E., \& Turk, D. C. (1981). Adaptation to divorce: Problems and coping strategies. Journal of Marriage and the Family, Feb., 179-189. 
Binder, L. M. (1983). Emotional problems after stroke. Stroke, 18, 1721.

Blazer, D., \& Williams, C. (1980). Epidemiology of dysphoria and depression in an elderly population. American Journal of Psychiatry, 137, 439-444.

Borgatta, E. F., \& Montgomery, R. J. V.(1987). Aging policy and societal values. In E. F. Borgatta and R. J. V. Montgomery (Eds.), , ritical issues in aging policy. Beverly Hills: Sage.

Bradburn, N. W. (1969). The structure of psychological well-being. Chicago: Aldine.

Brocklehurst, J.C., Morris, P., Andrews, K., Richards, B., Laycock, P. (1981). Social effects of stroke. Social Science and Medicine, $15 \mathrm{~A}, 35-39$.

Brody, E.M. (1981). 'Women in the middle' and family help to older people. The Gerontologist, 21 (5), 471-479.

Brody, E.M. (1985). Parent care as a normative family stress. The Gerontologist, 25(1), 19-29.

Brody, E.M., Schoonover, C.B. (1986). Patterns of parent care when adult daughters work and when they do not. The Gerontologist, $\underline{26}(4), 372-381$.

Burgess, A. W., \& Holmstrom, L. L. (1979). Adaptive strategies and recovery from rape. American Journal of Psychiatry, 136, 127882.

Cantor, M.H. (1979). Neighbors and friends: An overlooked resource in the informal support system. Research on Aging, 1, 434-463.

Cantor, M. H. (1980). The informal support system: Its relevance in the lives of the elderly. In E. F. Borgatta and N. G. McCluskey (Ed), Aging and society: Current research and policy perspectives(pp. 131-144). Beverly Hitls: Sage.

Cantor, M. H. (1983). Strain among caregivers: A study of experience in the United States. The Gerontologist, 23(6), 597-604.

Caplan, L. R. (1987). A general therapeutic perspective on stroke treatment. In R.E. Dunkle \& J.W. Schmidley (Eds.), Stroke in the elderly. New York: Springer.

Cicirelli, V. G. (1983). Adult children and their elderly parents. In T. H. Brubaker (Ed.), Family relationships in later Life. Beverly Hills: Sage Publications. 
Cicirelli, V. G. (1981a). Helping elderly parents: The role of adult children. Boston: Auburn House.

Clark, N. M. \& Rakowski, W. (1983). Family caregivers of older adults: Improving helping skills. The Gerontologist, 23(6), 637-642. Cohen, C. (1978). Three-year fol Tow-up study of stroke patients at the Medical College of Virginia. Southern Medical Journal, 71, 930-932.

Cohen, L. H., Towbes, L. C., Flocco, R. (1988). Effects of induced mood on self-reported life events and perceived and received social support. Journal of Personality and Social Psychology, 55 $(4), 669-674$.

Comstock, G. W., \& Helsing, K. J. (1976). Symptoms of depression in two communities. Psychological Medicine, 6 , 551-563.

Coppel, D. B., Burton, C. B., Becker, J. \& Fiore, J. (1985). Relationships of cognitions associated with coping reactions to depression in spousal caregivers of Alzheimer's disease patients. Cognitive Therapy and Research, 9, 253-266.

Coughlan, A.K., and Humphrey, M. (1982). Presenile stroke: Long-term outcomes for patients and their families. Rheumatology and Rehabilitation, 21, 115-122.

Crossman, L., London, C., Barrie, C. (1981). 01der women caring for disabled spouses: A model for supportive services. The Gerontologist, 21, 464-470.

DeJong, G., and Branch, L.G. (1982). Predicting the stroke patient's ability to live independently. Stroke, 13, 648-655.

Diener, E. (1984). Subjective well-being. Psychological Bulletin, 95, 542-575.

Dyken, M.L. (1987). Symptoms, epidemiology, and risk factors. In R.E. Dunkle \& J.W. Schmidley (Eds.). Stroke in the elderly. New York" Springer.

Enright, R.E. and Friss, L. (1987). Employed caregivers of brainimpaired adults: An assessment of the duat role. Finat report submitted to the Gerontological Society of America.

Fengler, A. P., \& Goodrich, N. (1979). Wives of elderly disabled men: The hidden patients. The Gerontologist, 19(2), 175-183.

Fiore, J., Becker, J., \& Coppel, D. (1983). Social network interactions: A buffer or a stress? American Journal of Community Psychology, 11, 423-39. 
Fitting, M., Rabins, P., Lucas, M. J., Eastham, J. (1986). Caregivers for dementia patients: A comparison of husbands and wives. The Gerontologist, 26 (3), 248-252.

Folkman, S. \& Lazarus, R. (1988). Coping as a mediator of emotion. Journal of Personality and Social Psychology, 54(3), 455-475.

Gallagher, D., \& Lovett, S (1987, November). Psychoeducational programs for reduction of stress in family caregivers. Paper presented at the annual meeting of the Gerontological Society of America, Chicago, IL.

Gallagher, D., Rose, J., Lovett, S., Silven, D. (1986, November). Prevalence, correlates and treatment of clinical depression in family caregivers. Paper presented at the annual meeting of the Gerontological Society of America, Chicago, IL.

Gallagher, D., Wrabetz, A., Lovett, S., Del Maestro, S., Rose, J. (in press). Depression and other negative affects in family caregivers. In E. Light \& B. Lebowitz (Eds.) Alzheimer's disease treatment and family stress: Directions for research. Washington, D. C.: U. S. Government Printing Office.

George, L. K. (1980). Role transitions in later life. Monterey, California: Brooks/Cole.

George, L. K. (1987). Easing caregiver burden: The role of informal and formal supports. In R. A. Ward and S. S. Tobin (Eds.), Heatth in aging: Sociological issues and policy directions. New York: Springer.

George, L. K., Gwyther, L. P. (1986). Caregiver well-being: A multidimensional examination of family caregivers of demented adults. The Gerontologist, $26(3), 253-259$.

Gibson, M. J., Family support patterns, policies and programs. In C. Nusberg (Ed.), Innovative aging programs abroad. Westport: Greenwood Press.

Gore, S. (1978). The effect of social support in moderating the health consequences of unemployment. Journal of Health and Social Behavior, 19, 157-165.

Gresham, G. E., Fitzpatrick, T. E., Wolf, P. A., McNamara, P. M., Kannel, W. B., \& Dawber, T. R. (1975). Residual disability in survivors of stroke - the Framingham study. The New England Journal of Medicine, 293, 954-956. 
Henley, S., Pettit, S., Todd-Pokropek, A., \& Tupper, A. (1985). Who goes home? Predictive factors in stroke recovery. Journal of Neurology, 33, 345-350. Holbrook, M. (1982). Stroke: Social and emotionatoutcome. Journal of the Royal College of Physicians of London. 12, 87-100.

Hooyman, N., Gonyea, J., \& Montgomery, R. (1985). The impact of inhome services termination on family caregivers. The Gerontologist, 25(2), 141-145.

Horowitz, A. (1985). Sons and daughters as caregivers to older parents: Differences in role performance and consequences. The Gerontologist, 25(6), 612-617.

House, J. S. (1974). Occupational stress and coronary heart disease: A review and theoretical integration. Journal of Health and Social Behavior.

House, J. S. (1981). Work stress and social support. Reading, MA: Addison-Wesley.

Hughes, S. L. (1985). Apples and oranges? A review of evaluations of community-based long-term care. Health Services Research, $20(4), 461-488$.

Hyman, M. D. (1971). The stigma of stroke. Geriatrics, 26, 132-141.

Jones, D.A., Vetter, N.J. (1984). A survey of those who care for the elderiy at home: Their problems and their needs. Social Science and Medicine, $19(5), 511-514$.

Johnson, C.L. (1983). Dyadic family relations and social support. The Gerontologist, 23, 377-383.

Johnson, C. L., \& Catalano, D. J. (1983). A longitudinal study of family supports to impaired elderly. The Gerontologist, $23(6)$, 612-618.

Kahan, J., Kemp, B., Staples, F. \& Brummel-Smith, K. (1985). Decreasing the burden in families caring for a relative with a dementing illness. Journal of the American Geriatrics Society, $\underline{33}, 664-670$.

Kane, R. L. \& Kane, R. A. (1985). A will and a way: What the United States can learn from Canada about caring for the elderly. New York: Columbia University Press.

Kaufman, S. (1988). Stroke rehabilitation and the negotiation of identity. In S. Reinharz \& G. Rowles (Eds.), Qualitative Gerontology. New York: Springer. 
Kaufman, S., Becker, G. (1986). Stroke: Health care on the periphery. Social Science and Medicine, 22(9), 983-989.

Kessler R.C., \& McLeod, J. (1985). Social support and psychological distress in community surveys. In $S$. Cohen \& L. Syme (Eds.) Social Support and Health. New York: Academic Press.

Kessler, R.C., Price, R.H., Wortman, C.B. (1985). Social factors in psychopathology: Stress, social support, and coping processes. In $M$. R. Rosenzweig and $L$. Porter (Eds.), Annual Review of Psychology. 36, 531-572.

Kinsella, G.J., and Duffy, F.D. (1980). Attitudes towards disability expressed by spouses of stroke patients. Scandinavian Journal of Rehabilitation Medicine, 12, 73-76.

Labi, M.L.C., Phillips, T.F., and Gresham, G.E. (1980). Psychosocial disability in physically restored long-term stroke survivors. Archives of Physical Medicine and Rehabilitation, 61, 561-565.

Lowy, L. (1983). Social policies and programs for the elderly as mechanisms of prevention. Aging and Prevention, 7-21.

Lewinsohn, P. M., Antonuccio, D. 0., Steinmetz, J., \& Teri, L. (1984) The coping with depression course: A psycho-educational intervention for unipolar dep ression. Eugene, OR: Castatia Publishing Co.

Lezak, M. (1978a). Living with the characterologically altered brain injured patient. Journal of Clinical Psychiatry, 39, 592-598.

Lezak, M. D. (1978b). Subtle sequelae of brain damage. American Journal of Physical Medicine, 57, 9-15.

Mahoney, F. I., \& Barthel, D. W. (1965). Functional evaluation: The Barthel Index. Maryland State Medical Journal, 14, 61-65.

McCubbin, H., Larsen, A., \& 01 son, D. (1981). F-COPES (Family crisisoriented personal evaluation scales) (measuring instrument). St. Paul, Minn: Family Social Science, University of Minnesota.

Montgomery, R.J.V., Stull, D.E., Borgatta, E.F. (1985). Measurement and the analys is of burden. Research on Aging, $7(1)$, 137-152.

Moroney, R. M. (1976). The family and the state: Considerations for social policy. London and New York: Longman Group.

Morycz, R. K. (1985). Caregiving strain and the desire to institutionalize family members with ATzheimer's disease: Possible predictors and model development. Research on Aging, $\underline{7}(3), 329-361$. 
National Center for Health Statistics. (1987). Health Statistics on 01 der Persons. Series 3, No. 25. Washington: Department of Human Services Publication (PHS) 87-1409.

Noh, S., Wood, D., \& Turner, R. (1984). Depression among the physically disabled: Somatic and psychological contributions. Paper presented at the American Public Health Association Meeting, Anahe im, CA.

Norusis, M. J. (1986). SPSS/PC+ for the IBM PC/XT/AT. Chicago: SPSS Inc.

Okun, M., Stock, W., Haring, M., \& Witter, R. (1984). Health and subjective well-being: A meta-analysis. International Journal of Aging and Human Development, 19 , 111-132.

Pagel, M. D., Erdly, W. W., \& Becker, J. (1987). Social networks; We get with (and in spite of) a little help from our friends. Journal of Personality and Social Psychology, 53(4), 793-804.

Pearl in, L.I., Lieberman, M.A., Menaghan, E.G., Mullan, J.T. (1981). The stress process. Journal of Health and Social Behavior, 22, 337-356.

Poulshock, S.W., Deimling, G.T. (1984). Families caring for elders in residence: Issues in the measurement of burden. Journal of Gerontology, 39 (2), 230-239.

Pratt, C., Schmall, V.L., Wright, S., Cleland, M. (1985). Burden and coping strategies of caregivers to Alzheimer's patients. Family Relations, 34, 27-33.

Pratt, C., Wright, S., Schmall, V. (1987). Burden, coping and health status: A comparison of family caregivers to community dwelling and institutionalized Alzheimer's patients. Journal of Gerontological Social Work, 99-112.

Price, T. R. (1987). Depression and stroke. In R. Dunkle \& J. W. Schmidley (Eds.). Stroke in the elderly. New York: Springer.

Radl off, L. S. (1977). The CES-D Scale: A self-report depression scale for research in the general population. Appl ied Psychological Measurement, $1,385-401$.

Ratcheson, R. A., \& Selman, W. R. (1987). Surgical therapy for stroke. In R. E. Dunkle \& J. W. Schmidley (Eds.), Stroke in the elderly. New York: Springer. 
Rau, M.T. (1986). Elderly stroke victims and their partners: A longitudinal study of social support and well-being changes associated with a disabling stroke. (Doctoral dissertation, Portland State University (1986.) Dissertation Abstracts International, $47,2274$.

Schulz, R., Tompkins, C. A., Wood, D., \& Decker, S. (1987). The social psychology of caregiving: Physical and psychological costs of providing support to the disabled. Journal of Applied Social Psychology, 17(4), 401-428.

Schulz, R., Tompkins, C. A., Rau, M. T.(1988). A longitudinal study of the psychosocial impact of stroke on primary support persons. Psychology and Aging, $\underline{3}(2), 131-141$.

Schulz, R. and Rau, M. T. (1984). Elderly stroke victims: The impact of a disabling stroke on the elderly and their social networks. Final Report submitted to the AARP-Andrus Foundation.

Szulc, T. (1988). How we can help ourselves age with dignity. Parade Magazine, May 29, 4-7.

Shanas, E. (1979a). Social myth as hypothesis: The case of the family relations of old people. The Gerontologist, 19, 3-9.

Shanas, E. (1979b). The family as a social support system in old age. The Gerontologist, 19, 169-174.

Silliman, R. A., Earp, J. L., Fletcher, R. H., Wagner, E. H. (1987). Stroke: The perspective of family caregivers. Journal of Applied Gerontology, $6(4), 363-371$.

Silliman, R.A., Fletcher, R.H., Earp, J.L., Wagner, E.H. (1986). Families of elderly stroke patients: Effects of home care. Journal of the American Geriatric Society, 34(9), 643-648.

Silver, R. L., \& Wortman, C. B. (1980). Coping with undesirable life events. in J Garber \& M. E. P. Seligman (Eds.), Human helplessness: Theory and applications (pp. 279-375). New York: Academic Press.

Silverstone, B. \& Horowitz, A. (1987). Issues of social support: The family and home care. In R.E. Dunkle \& J.W. Schmidley (Eds.), Stroke in the elderly. New York: Springer.

Simons, R.L., West, G.E. (1985). Life changes, coping resources, and health among the elderly. International Journal of Aging and Human Development, $20(3), 173-179$.

Soldo, B.J., \& Myllyluoma, J. (1983). Caregivers who live with dependent elderly. The Gerontologist, $23,(6), 605-611$. 
Springer, D., \& Brubaker, T. H. (1984). Family caregivers and dependent elderly: Minimizing stress and maximizing independence. Beverly HiTls: Sage.

SPSSX Users Guide, 2nd Edition. 1986. New York:McGraw Hill, Inc.

Stein, R. W., Hier, D. R., and Caplan, L. R. (1985). Cognitive and behavioral deficits after right hemisphere stroke. Stroke, 20, $1-5$.

Stephens, M. A. P., Kinney, J. M., Norris, V. K., Ritchie, S. W. (1987). Social networks as assets and liabilities in recovery from stroke by geriatric patients. Psychology and Aging, 2(2), 125-129.

Stephens, S.A., \& Christianson, J.B. (1987). Informal care of the elderly. Lexington, Massachusetts: D.C. Heath and Co.

Stoller, E.P. (1985). Elder-caregiver relationships in shared households. Research on Aging, $7,(2), 175-193$.

Stone, R. (1987). Exploding the myths: Caregiving in America. A study by the Subcommittee on Human Services of the Select Committee on Aging, House of Representatives. Committee Publication No. 99611. Washington: U. S. Government Printing Office.

Stone, R., Cafferata, G., \& Sangl, J. (1987). Caregivers of the frail elderly: A national profile. The Gerontologist, 27(5), 616-626.

Tangeman, P., Banaitis, D., Williams, A., \& Wingfield, D. (1987). Physical and psychosocial rehabilitation potential of chronic stroke patients. Paper presented at the 40th annual meeting of the Gerontological Society of America, Washington, D.C.

Thoits, P. A. (1983). Dimensions of life events that influence psychological distress: An evaluation and synthesis of the literature. In H. B. Kaplan (Ed.), Psychosocial stress: Trends in theory and research (pp. 33-103). New York: Academic Press.

Thoits, P. A. (1985). Social support and psychological well-being: Theoretical possibilities. In I. Sarason and B. Sarason (Eds.), Social support: Theory, research, and applications. Boston: Martinus Nijhoff.

Thompson, L.W., Gallagher, D., \& Breckenridge, J.S. (1987). Comparative effectiveness of psychotherapies for depressed elders. Journal of Consulting and Clinical Psychology, 55(3), 385-390.

Tompkins, C. A., Schulz, R., Rau, M. T. (1988). Post-stroke depression in primary support persons: Predicting those at risk. Journal of Consulting and Clinical Psychology, 56(4), 502-508. 
Unks, R. P. (1983). The relative influence of social, physical, and psychological factors on the morale and life satisfaction of elderly wives of stroke patients: A descriptive exploratory study. (Doctoral dissertation, University of Washington, 1983). Dissertation Abstracts International, 44, 2585.

Vachon, M. L. S., Rogers, J., Lyall, W. A. L., Lancee, W. J., Sheldon, A. R., \& Freeman, S. J. J. (1982a). Predictors and correlates of adaptation to conjugal bereavement. American Journal of Psychiatry, 139, 998-1002.

Vachon, M. L. S., Sheldon, A. R., Lancee, W. J., Lyall, W. A. L., Rogers, J., \& Freeman, S. J. J. (1982). Correlates of enduring stress patterns following bereavement: Social network, life situation and personality. Psychological Medicine, 12, 783-788.

VanWinkle, N. W. (1987). Depressive symptomatology of spouses and children caring for a relative with dementia. Paper presented at the 40th annual scientific meeting of th Gerontological Society of America, Washington, D. C.

Veroff, J., Douvan, E., Kulka, R. (1981). The inner American. New York: Basic Books.

Videka-Sherman, L. (1982). Coping with the death of a child: A study over time. American Journal of Orthopsychiatry, 52 (4), 688-698.

Wade, D. T., Legh-Smith, J., \& Hewer, R. L. (1986). Effects of living with and looking after survivors of stroke. British Medical Journal, 293, 16 August, 418-420.

Weinfeld, F.D. (Ed.) (1981). The national survey of stroke. Stroke, 12 (Part 2, Supplement1).

Williams, A.K. (1985). Physical illness and depression: Changes over time in middle aged and elderly persons. (Doctoral Dissertation, Portland State University (1985). Dissertation Abstracts International, $46,2585$.

Whitney, F. W. (1987). Using physical and neuropsychological assessment in the nursing care of the acute stroke patient. In R.E. Dunkle \& J.W. Schmidley (Eds.), Stroke in the elderly. New York: Springer.

Zarit, S.H., Reever, K.E., and Bach-Peterson, J. (1980). Relatives of the impaired elderly: Correlates of feelings of burden. The Gerontologist, 20, 649-655.

Zarit, S.H., Todd, P.A., Zarit, J.A. (1986). Subjective burden of husbands and wives as caregivers: A longitudinal study. The Gerontologist, 26(3), 260-266. 
APPENDIX A

RECRUITMENT LETTER 


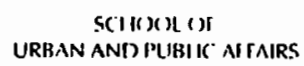

INSIITUTE ONACINC;

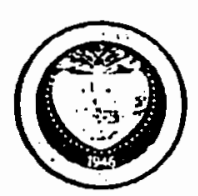

P(JRILANI)

SIAIE

UNIVERSIIY

P.(). BOX -51

P(ORIL ANI), OREC ION

9:20:

$503 / 224.4 \div 30$

June 1987

Dear Friend,

About two years ago you participated in the Stroke Study conducted by the Institute on Aging at Portland State University. The study provided valuable information about the effects of stroke on the lives of stroke patients and their families during the first year following an initial stroke.

The long term effects of stroke, and adjustment to them, are often just as problematic as the early effects. Not much is known about living with the effects of stroke over a longer period of time. In order to find out more about this from people who have already shared their initial experience and response to stroke, we are conducting a follow-up interview by telephone during the summer.

The telephone interview will be about a half hour long. and will be done with just the family member or friend who participated in the first study (the support person] rather than with both the support person and the person who had the stroke. The purpose of this follow-up interview is to learn about the specific needs and concerns of those who provide care and support for people who have had strokes.

I hope you will be willing to participate. I will call you within the next few weeks to answer any questions you may have about the study and to set a convenient time for the interview. All information is confidential and will never be identified with you by name. Of course you have the right to refuse to participate.

If you have any questions, please call me at the Institute on Aging, Portland State University. The phone number is 229-3952. You may also talk with Dr. Nancy Chapman. Acting Director of the Institute on Aging, about the study.

I look forward to talking with you.

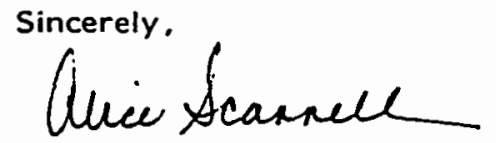

Alice Scannell 
APPENDIX B

INFORMED CONSENT

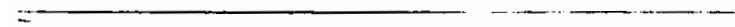




\title{
STROKE PROJECT
}

\author{
PORTLAND STATE UNIVERSITY INSTITUTE ON AFING
}

\author{
Informed Consent Form \\ (Primary Informant)
}

I have been asked to particlpate in the study, "Soclal Network and Hell-Being Changes Caused by a Disabling Stroke", which is being conducted by Richard Schulz, Ph. D., Principal Investigator, and Marie T. Rau, Profect Director, of the Institute on AgIng at Portland State University.

The purpose of the study is to learn more about the social support systems of persons who have had a stroke and their familles, how the nature and amount of support may change over time, and hoir these changes in social support are related to the sense of wellbeing experienced by stroke victims and their families. It is hoped that this research will help us to learn more about the psycho-social aspects of stroke, and what role an individual's social support system plays in recovery from a stroke.

I $w 111$ be asked to respond three times to a questionnaire about those persons and types of social support which are important in my IIfe. There will also be some questions regarding oy current health and life situation as I view It. I understand that I will be interviewed in person or by tejephone, and that these interviews will take place in my home or in the hospital if inperson. I will be reimbursed for mileage costs which might result from the completionrof these interviews. The first interview will take place 3 to six weeks after my stroke. The second interview will be held 6 months after the first interview, and the third interview will take place 6 months after the second interview. Each interview should take about an hour of my time.

I. also understand that my who has suffered the stroke may be asked to respond to some of the same, or similar questions. I agree to allow persons conducting this research to obtain certain information regarding my from his/her physlcian, theraplsts, and/or frommedical records, with his/her physician's approval. I understand that at all times our right to privacy regarding any of the study information will be strictly safeguarded and protected. Nelther I nor my will be personally identified on any of the questionnalreforms, in any of the data analysis, or in any published reports of the study.

I have been assured that participation in this study should involve little risk or discomfort. I may discontinue my participation in the study at any time if $I$ find any part of the procedure to be uncomfortable or objectionable. I understand that elther Dr Schulz or Mrs. Rau (Institute on Aging, phone: 229-3952) w11l be happy to answer any questions I may have about the profect. 
Informed Consent (P. I.) 2 .

I understand that I am free to refuse to participate or to withdraw from participation in this study at any time.

I have carefully read or had read to me and understand the foregoing. I hereby voluntarily consent to my participation $n$ the experimental procedures as described above.

Name of Subject

Signature of Subject Date

Name of Witness

Signature of Witness Date

Name of Person obtaining Signatures 
APPENDIX C

TIME 4 INTERVIEW 
Stroke Study Time 4 Telephone Interview

THE PSYCHOSOCIAL IMPACTS OF CAREGIVING

ON THE PRIMARY SUPPORT PERSONS OF STROKE VICTIMS

Dyad I.D.\#

Interviewer

Date

Time begin

Time end

IF STROKE VICTIM IS DECEASED, ENTER DATE OF DEATH:

(EDITOR: calculate \# of months between T3 interview and date of SV's death $199=$ na) Also calculate \# of months between SV's death and T4

interview

$$
\text { (99 = na) }
$$

Thank you very much for taking the time to talk with me. As you know, this interview will take about 30 minutes, so if you need to take a break or get a cup of coffee, please let me know. All of the information you share is confidential, and your answers will never be ident if ied with you by name.

Some of the questions in the interview will ask about changes since we talked with you last time. Our last interview with you was on

INTERVIEWER NOTE: SKIP ALL QUESTIONS MARKED WITH AN * IF STROKE VICTIM IS DECEASED.

The first set of questions are for general background information.

1. What is your age now?

*2. What is 's (sv's) age now? 
Stroke Study Time 4 Telephone Interview

3. Has there been a change in your marital status since the last interview?

$$
\begin{array}{ll}
0=\text { no } & 5=\text { divorced } \\
1=\text { living as married } & 6=\text { living alone } \\
2=\text { married } & 8=\text { refused } / \mathrm{dk} \\
3=\text { widowed } & 9=\mathrm{mi} \\
4=\text { separated } &
\end{array}
$$

4. Have there been any changes in your employment since the last interview?

$$
0=\text { no }(\text { CO TO Q. \#7) } 1 \text { = yes }
$$

5. Was your employment change triggered by the stroke?

$$
\begin{array}{ll}
0=\text { no }(\mathrm{CO} \text { To Q. } 7) & 1=\text { yes } \\
& 9=\mathrm{na} / \mathrm{mi}
\end{array}
$$

6. Why did you change employment? (ASK AS OPENENDED--INIERVIEWER CODE).

$1=$ Needed to have more time to care for the stroke vict im

$2=$ Needed to replace a regular income which was lost due to the stroke

$3=$ Needed to have more money for medical expenses

4 = other

$$
9=\mathbf{n a} / \mathrm{mi}
$$

7. Has your standard of living (economically) changed since the last interview?

$$
0=\text { no }(C O \text { To } Q \text {. \#9) } 1=\text { yes } \quad 8=\mathrm{dk} / \mathrm{ref} \quad 9=\mathrm{na} / \mathrm{mi}
$$

8. Is it now better or worse?

$$
0=\text { worse } \quad 1=\text { better } \quad 8=\mathrm{dk} / \mathrm{ref} \quad 9=\mathrm{na} / \mathrm{mi}
$$

9. Have you changed your residence since the last interview?

$$
0=\text { no }(\operatorname{CO} \text { To } Q \text {. \#12) } 1 \text { = yes }
$$

10. Was your move triggered by the stroke?

$$
0=\text { no } \quad 1=\text { yes } \quad 9=\mathrm{na} / \mathrm{mi}
$$


Stroke Study Time 4 Telephone Interview

11. Why did you move? (Ask as openended quest ion; interviewer circle as many as apply. RECORD VERBATIM ANSWER FOR "other".)

$$
\begin{aligned}
& 1=\text { we needed a smaller place, easier to take care of } \\
& 2=\text { we needed a less expensive place } \\
& 3 \text { = stroke victim needed a place which was easier to use and get around in } \\
& 4=\text { we wanted/needed more services } \\
& 5=\text { other }
\end{aligned}
$$

$8=\mathrm{refused} / \mathrm{dk}$

$9=\mathrm{na} / \mathrm{mi}$

HEALTH STATUS

Now I am going to ask a few questions about your heal th:

12. Do you have any chronic health problems such as diabetes, heart disease, high blood pressure, arthritis, or any other condition?

LIST:

Total Number Health Probs.

13. Have any of these come about since the stroke?

Total number since stroke $9=$ na

14. Have you been hospitalized in the last year?

$$
0=\text { no }(\text { GO To } Q \text {. } \$ 17) \quad 1=\text { yes }
$$

15. How many times?

$$
9=n a
$$


Stroke Study Time 4 Telephone Interview

16. Why were you hospital ized? (RECORD VERRATIM RESPONSE; EDITOR CODE)

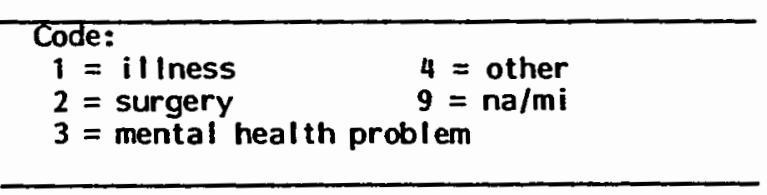

17. About how many times have you visited the doctor in the past year, other than for regular checkups?

\# visits for medical problems

visits for mental heal th problems

18. How would you rate your overall health at the present time? Would you say it is (READ CHOICES)

$$
\begin{array}{ll}
1=\text { excel lent } & 5=\text { poor } \\
2=\text { very good } & 8=\text { refused } / \mathrm{dk} \\
3=\text { good } & 9=\mathrm{mi} / \mathrm{na} \\
4=\text { fair } &
\end{array}
$$

19. Is your heal th now better, about the same, or worse since the last interview?
1 = better
2 = same
$8=\mathrm{refused} / \mathrm{dk}$
$3=$ worse
$9=\mathrm{mi} / \mathrm{na}$ 
Stroke Study Time 4 Telephone Interview

STROKE--PERCEPTIONS AND FEELINCS

(IF SV IS DECEASED, CO TO Q. $\$ 35$ )

*Now I would like to ask some questions about things related to the stroke.

's (SV's) health and other

^20. 's (SV's) residence: (Interviewer code. Ask directly only if not already known].

$1=1$ ives alone in own home/apartment

$2=$ lives with primary support person

$3=$ with another family member or companion

4 = foster home or licensed care facility+ ( $+=$ if yes, ask Q. 21)

$5=$ is in hospital

$6=$ other (specify)

$9=\mathrm{na} / \mathrm{mi}$

*21. Ask only if $S V$ is in a foster home or licensed care facility: What were the circumstances that led to 's move to a foster home (or I icensed care facility?) RECORD VERBATIM RESPONSE; EDITOR COOE)

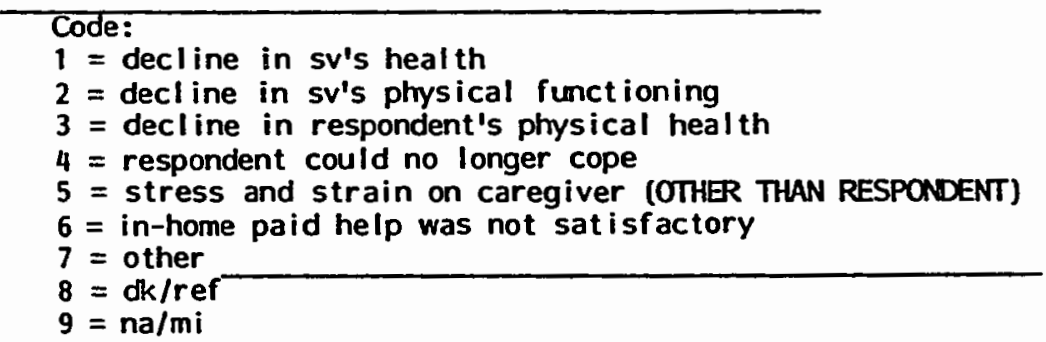

*22. Has had any other strokes since the first one?

$0=$ no (CO TO Q. 25$) \quad 1=$ yes

How many?

Number of additional strokes $\overline{9}=$ na

*23. How many $t$ imes was hospitalized for those strokes? Number of hospitalizations 
Stroke Study Time 4 Telephone Interview

*24. What kind of physical or functional changes occurred with the additional strokes? (RECORD VERBATIM RESPONSE; EDITOR CODE)

Code:
$1=$ decreased functional capacity
$2=$ speech af fected
$3=$ negat ive personal ity/behavior changes
$4=$ positive personal i ty/behavior changes
$5=$ other
$9=$ na/mi

*25. At this time, are you concerned about the possibility of another stroke?

$$
0=\text { no }(C O \text { To Q. \#27) } 1 \text { yes } 8=\mathrm{ref} / \mathrm{ok} \quad 9=\mathrm{na} / \mathrm{mi}
$$

*26. Are you (READ CHOICES)

$$
\begin{aligned}
& 1=\text { a little concerned } \\
& 2=\text { moderately concerned } \\
& 3=\text { very concerned } \\
& 9=\mathrm{na} / \mathrm{mi}
\end{aligned}
$$

*27. What other chronic illnesses such as diabetes, heart

disease, high blood pressure, arthritis, or other health problems does (SV) have at this time?

Total number SV heal th problems

*28. Has been hospitalized in the past year?

$$
0=\text { no }(\text { GO TO Q. } \$ 31) \quad 1=\text { yes }
$$

*29. How many $t$ imes?

$$
\overline{9}=\mathrm{na}
$$


Stroke Study Time 4 Telephone Interview

*30. Why was hospitalized? (RECOFO VERBATIM RESPONSE; EDITOR COOE)

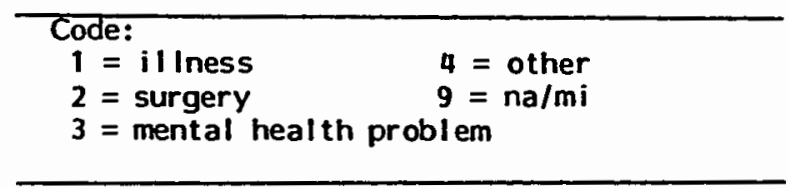

*31. In your view, is 's health now better, about the same, or worse since the last interview?
1 = better
2 = same
$8=\mathrm{refused} / \mathrm{dk}$
$3=$ worse
$9=\mathrm{mi} / \mathrm{na}$

*32. In your view, in terms of needing help. how severe are heal th problems now? Would you say they are (READ CHOICES)

's physical

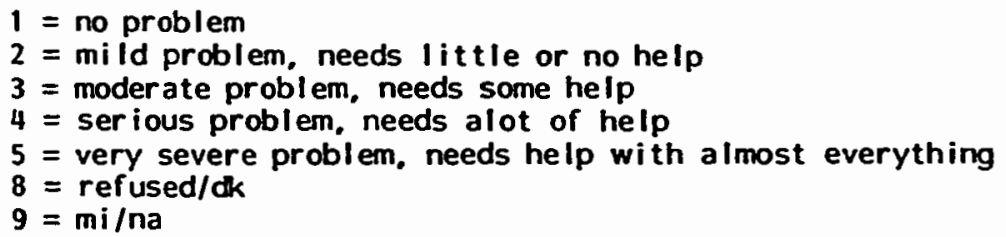

*33. The next 2 questions are about 's ability to

communicate with you personally and with others. Tell me which of the following statements best describes 's communication with you: (READ CHOICES)

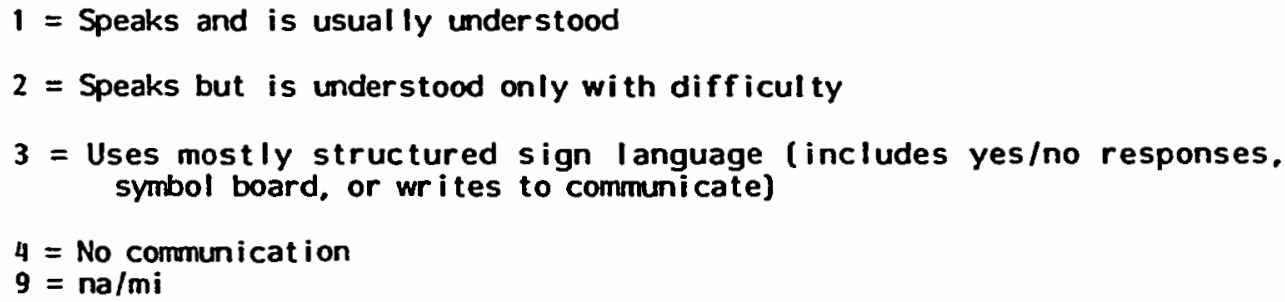

*34. How about 's communication with other people?

(CODE AS IN Q. \#33) 
Stroke Study Time 4 Telephone Interview

\section{CAREGIVER ROLE}

Now l'd like to ask some questions about your role as a support person for someone who had a stroke.

IF STROKE VICTIM IS DECEASED, ASK QUESTIONS 35-48 IN THE FOLLOWING MANNER: "WHEN YOU WERE LAST CARING FOR MEMBERS HELPED YOU CARE FOR

DID YOU......". "ABOUT HOW MANY FAMILY ON A REQLAR BASIS", etc.

35. I have a $l$ ist of different kinds of assistance which people who have had strokes may need. As I read each item, I'd like you to tell me if is (was) able to do this for him/herself, if you do (did) it and if someone else (such as an agency, other family member. friend, or private arrangement) he lps (helped) with that task. As we do each one, please also tell me how of ten each of you do (did) these things.
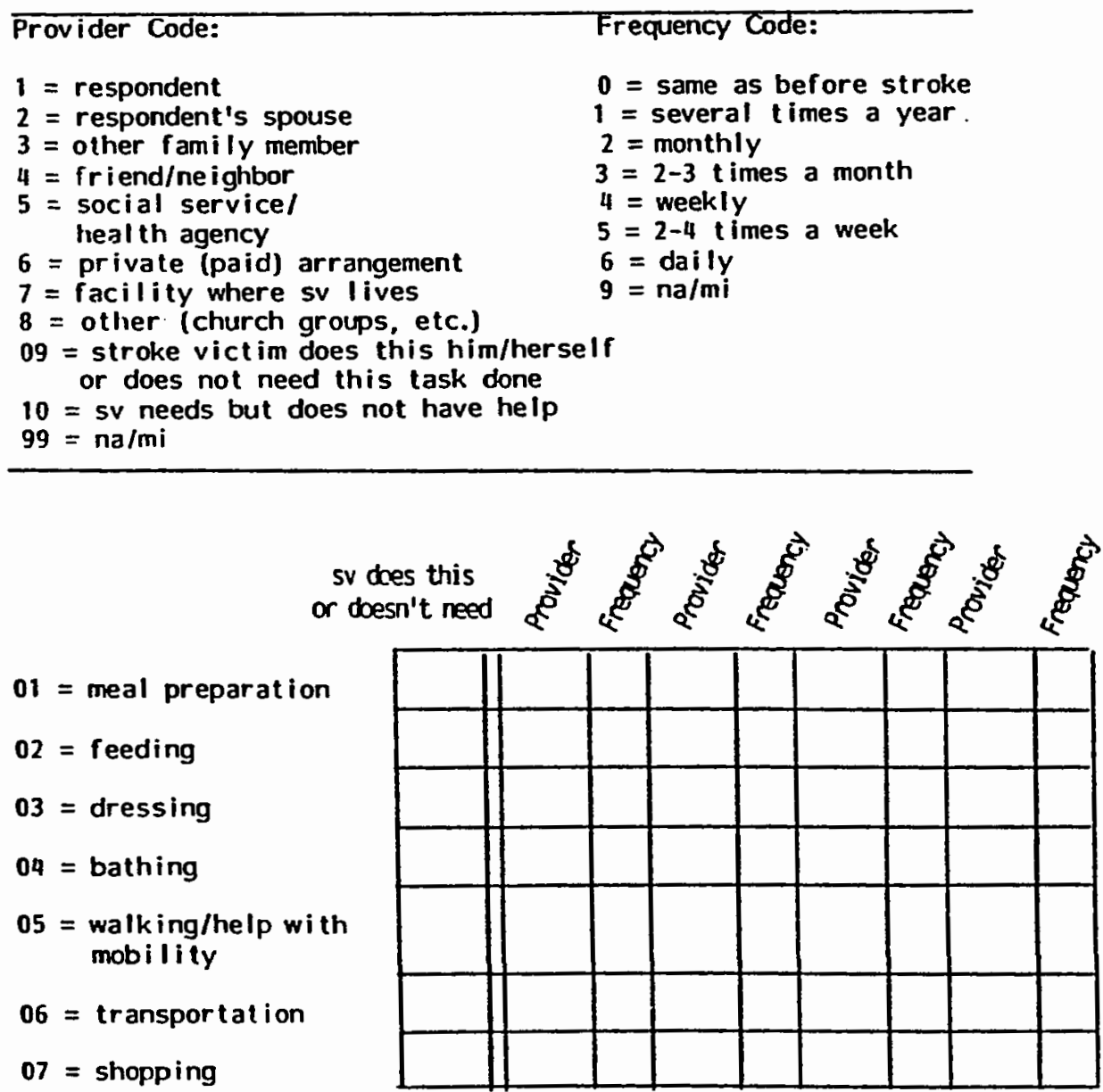
Stroke Study Time 4 Telephone Interview
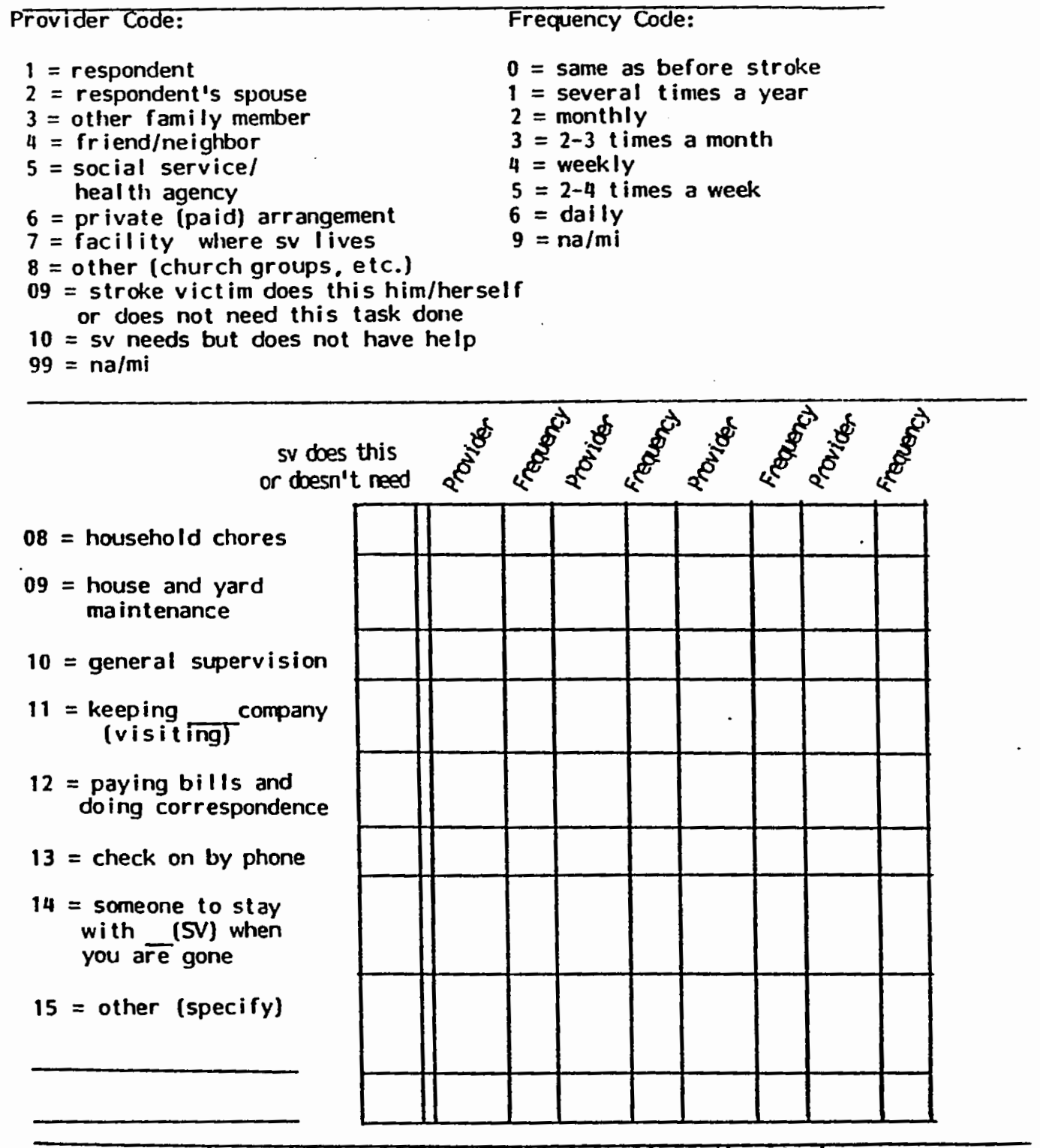

Editor Compute: Total tasks needing assistance since the stroke

Total tasks done by respondent (provider 11)

Total tasks done by informal system (provider $2,3,4,6.7$ )

Total tasks done by formal service system (provider (5) 
Stroke Study Time 4 Telephone Interview

(INTERVIEWER NOTE: IF SV HAS BEEN DECEASED FOR MORE THAN ONE YEAR, SKIP QUESTIONS 36-40)

36. About how many family members have helped in the past year

on a regular basis?

$$
9=\text { na }
$$

37. About how many friends and neighbors have regularly helped you do things for in the past year? $9=n a$

38. About how many social service or health agencies have helped you in the past year? 9= na

39. In the past year, did you seek information about services or benefits for or make arrangements for benef its. services, or ass istance on s behalf? (Probe: such as veterans benefits, social and health services, transportation, etc.)

$$
\begin{aligned}
& 0=\text { no ( } \mathrm{GO} \text { To Q. \# 4i) } 8=\mathrm{dk} / \mathrm{ref} \\
& 1=\text { yes } \quad 9=\mathrm{na} / \mathrm{mi}
\end{aligned}
$$

40. About how of ten in the past year have you sought information or made arrangements for services or benef $i$ ts on behalf of

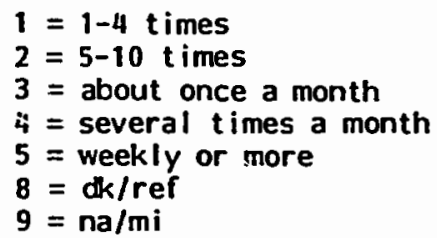

41. On a scale from one to five, with one being not very difficult and five being very difficult, how hard would you say it is (was) overall for you to get helpful inf ormation or to arrange for benefits, services, or assistance after the stroke? 2 (not very difficult)

4 5 (very difficult) 
Stroke Study Time 4 Telephone Interview

42. What are (were) some of the difficulties? (RECORD VERBATIM RESPONSE: EDITOR CODE)

Code:
$00=$ none/no response/don't know
$01=$ didn't know what quest ions to ask
$02=i t$ takes awhile to know what services are available
$03=5 v$ is critical of paid care providers--they won't stay
$04=5 v$ is critical of paid care providers--insists I find someone else
$05=5 v$ refuses help from outsiders
$06=$ services are too expensive to have on a regular basis
$07=$ there are waiting lists for the services I need/ too much red tape
$08=0$ other
$99=$ na/mi


Stroke Study Time 4 Telephone Interview

PERCEIVED STRESS

43. Sometimes people who help others worry about getting sufficient help or assistance for them. On a scale from one to five, with one being not at all and five being quite alot, how much would you say you worry about get $t$ ing suf $\mathrm{f}$ cient help for

$\begin{array}{lllll}1 & 2 & 3 & 4 & { }^{5} \\ \text { (not at all) } & & & & \text { (quite alot) }\end{array}$

44. I'm going to mention several areas in which people who help others sometimes experience stress or strain. On a scale of 1 to 5 , with 1 being little or no strain and 5 being a great deal of strain, how much physical strain do (did) you experience overall in caring for TERMS OF NOW RATHER THAN BEFORE PLACENENT) ? IIF SV IS IN LTC FACILITY. ASK IN
1
2
3
4
5

45. Again. on the scale of 1 to 5. how much emotional strain do (did) you experience?
1
2
3
4
5

46. How much financial strain do (did) you feel (on the scale of 1 to 5 )?

4

5

*47. About how many times in an average week is your sleep interrupted because you have to get up to take care of ?

t imes per week

$0=$ less than once a week
$8=\mathrm{dk} / \mathrm{ref}$
$9=\mathrm{na} / \mathrm{mi}$ 
Stroke Study Time 4 Telephone Interview

48. People respond to new roles in many different ways. I'd I ike you to indicate how much the following statements describe the things you have done

(did) to adjust to the role of supporting af ter the stroke. As I read

the items, think about yourself and tell me how of ten you cope in these ways. The choices are somet imes, of ten, or not at all.

Code:

$0=$ not at all $8=d k /$ ref

$1=$ sometimes, a little $\quad 9=\mathrm{na} / \mathrm{mi}$

2 = often, alot

(Interviewer write appropriate code for each response.)

When I have faced problems or difficulties I have responded by:

a. sharing my difficulties with relatives

b. seeking support and encouragement from friends

c. attending church services

d. seeking information and advice from persons in other families who have faced the same or similar problems

e. seeking advice from relatives

f. participating in church activities

9. seeking assistance from community agencies and programs designed to help families in our situation

h. sharing concerns with close friends

i. seeking advice from a minister

j. making sure $\mid$ have some time to do things just for myself

k. joining a caregiver support group

1. having faith in Cod

m. taking a caregiver training course

n. seeking advice from our physician

o. using relaxation techniques or exercise 
Stroke Study Time 4 Telephone Interview

49. Are there other ways in which you have coped that were not on the list? (1f SV is severely impaired, probe: such as temporarily placing in a nursing home so you can get a rest, vacation, etc.? Ask as openended question. RECORD VERATIM RESPONSE FOR "OTHER".

$$
0=\text { no }
$$

1 = ask family to take sv for periods of time

$2=$ place sv in nursing home for short periods so 1 can go away/get rest

$3=$ other

*50. In general, how well do you and CHOICES)

(sv) get along with each other? (READ

$$
\begin{aligned}
& 3=\text { very well } \\
& 2=\text { fairly well } \\
& 1=\text { not too well }
\end{aligned}
$$

*51. Are you concerned that you might not be able to care for in the future?

$$
0=\text { no }(\text { CO TOQ. } \$ 53) \quad 1 \text { = yes }
$$

^52. Are you (READ CHOICES)

$$
\begin{array}{ll}
1=\text { a little concerned } & \\
2=\text { moderately concerned } & 8=\mathrm{refused} / \mathrm{dk} \\
3=\text { very concerned } & 9=\mathrm{na} / \mathrm{mi}
\end{array}
$$

*53. Do you consider___ (sv) as being dependent on you now for care or support because of the stroke?

$$
0=\text { no } \quad 1=\text { yes } \quad 8=\mathrm{ok} / \mathrm{ref} \quad 9=\mathrm{na} / \mathrm{mi}
$$

*54. Do you think of yourself as a caregiver for (RECORD VERBATIM COMMENTS]

$$
0=\text { no } \quad 1=\text { somet imes but not always } 2=\text { yes } \quad 9=\mathrm{na} / \mathrm{mi}
$$


Stroke Study Time 4 Telephone Interview

SOCIAL SUPPORT

You may remember that in the previous interviews we asked you to name the friends, neighbors, or relatives who provided help or support to you after the stroke. I'd like you to think about the people who have been supportive to you during the past year--either in terms of moral support, helpful tasks. transportation or of fering assistance. I will ask only a few questions about them now.

55. Thinking about the last year, when you wanted to talk with someone about personal matters, with whom did you talk? Please give me just the ir first name and their relationship to you. They might include friends, neighbors, your spouse, relatives, or others whom you know.

Relationship code:

1 = spouse

2 = child

$3=$ sibling

4 = parent/parent-in-I aw

$5=$ other relative
$6=$ neighbor
$7=$ work associate
8 = friend
0 = other

Name

Relat.

Total

56. Are there people who would come to you to talk about something they are worried about, or about a personal matter? Again, these can be relatives. friends, your spouse, or others. Name

Relat.

Total

Total reciprocal (named both $55 / 56$ ) 
Stroke Study Time 4 Telephone Interview

\begin{tabular}{ll}
\hline Relationship code: & \\
$1=$ spouse & $6=$ neighbor \\
$2=$ child & $7=$ work associate \\
$3=$ sibling & $8=$ friend \\
$4=$ parent/parent - in-law & $0=$ other \\
$5=$ other relative & \\
\hline
\end{tabular}

57. Are there people you would ask to assist you with transportation, chores, cooking, or who would provide occasional time off for you if you needed such help?

Name Relat.

58. Do you provide this type of help for others? For whom do you provide help? Name

Relat.

Total *

Total

Total \# reciprocal (named both $57 / 58$ )

59. Are there people who are dependent on you for care or emotional support? (Probe--such as children, grandchildren, or elderly relatives?) (INTERVIEWER ASK ACE FOR EACH PERSON NAMED; INCLUE SV IF DEPENDENT)
Name
Relat. Age

Total under 18

Total $* 19-65$

Total $: 66-79$

Total 80 plus 
Stroke Study Time 4 Telephone Interview

60. In general, how sat isfied are you with the amount of social contact you have with others at this time?

$$
\begin{array}{ll}
1=\text { very dissat isfied } & 5=\text { very sat isfied } \\
2=\text { somewhat dissat isfied } & 8=\text { refused } \\
3=\text { neutral } & 9=0 \mathrm{k} / \mathrm{mi} / \mathrm{na} \\
4=\text { somewhat satisfied } &
\end{array}
$$

61. In general, how satisfied are you with the quality or closeness of the social contact you have with others at this $t$ ime?

$$
\begin{array}{ll}
1=\text { very dissat isfied } & 5=\text { very sat isfied } \\
2=\text { somewhat dissat isf ied } & 8=\text { refused } \\
3=\text { neutral } & 9=0 \mathrm{k} / \mathrm{mi} / \mathrm{na} \\
4=\text { somewhat sat isfied } &
\end{array}
$$

62. Have there been any good or bad events in your Iife in the past year? Events such as family celebrations or crises. loss of friends or family through death or moving away, vacations or other important events? (Ask as openended quest ion; circle all that apply. RECORD VERBATIM RESPONSE FOR 'OTHER'.

$00=$ no

01 = death or inst itut ionalization of spouse

$02=$ loss of close friend through death, institutionalization, or moving away

03 = divorce/ pending divorce

$04=$ death or loss of immediate family member

$05=$ loss of paid service

$06=$ own heal th problems

$07=$ health problems of spouse or immediate family member

08 = moving

$09=$ vacat ion

$10=$ family celebrations

11 = heal th improvement

12 = marriage (self)

$13=$ other (specify):

Total \# good events

Total bad events 
Stroke Study Time 4 Telephone Interview

*63. What is your greatest worry or concern at this time related to the stroke? (Ask as openended; RECORD VERBATIM ANSWERS. Editor code: use right marg in to I ist all that apply.)

\section{EDITOR CODE:}

$00=$ don't know, nothing, no response

$01=$ another stroke

$02=$ respondent's own health and well-being as result of burden/responsibility for $\mathbf{S V}$

03 = expectations for sv's recovery

$04=S V$ independence, mobility, ability to care for self

$05=$ SV physical well-being, health problems

$06=5 V$ mental health (depression, etc.)

$07=\mathrm{SV}$ commmicat ion problems

$08=S V$ quality of I ife

09 = $\mathrm{f}$ inancial concerns

$10=$ help with repairs, etc.

11 = other

12 = quality of care if sv needs institutional placement

$99=$ na

*64. Do you have other worries or concerns related to the stroke?

(IF YES, RECORD VERBATIM RESPONSE AND CODE AS IN Q. \#63.) 
Stroke Study Time 4 Telephone Interview

65. In general, what obstacles or difficulties have you found in providing help and support for Editor Code.) since the stroke? (RECORD VERBATIM RESPONSE. Probe.

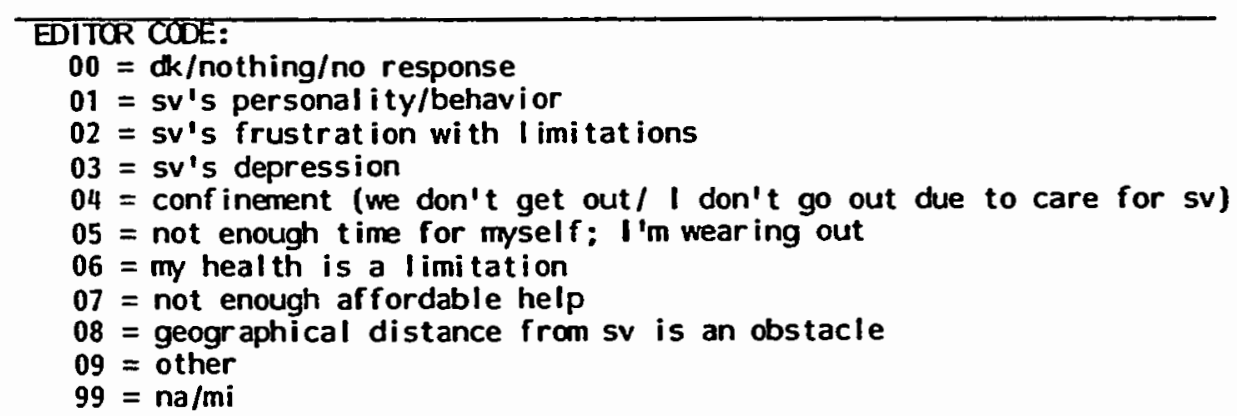

*66. Overall, do you feel that you have adjusted to the stroke? (CIRCIE APPROPRIATE CATECORY; PROBE FOR QARIFICATION AND RECORD VERBTIM REPONSE. FOR EXAMPLE, IF "NO" ANSWER, ASK WHAT ARE SOME THINCS THAT HAVE MADE ADJUSTMENT DIFFICULT? IF "YES" ANSWER, ASK WHAT HAS HELPED YOU TO ADJUST?)

$0=$ no/not quite $1=$ somewhat $2=$ pretty well $3=$ very well

Reasons: 
Stroke Study Time 4 Telephone Interview

*67. Do you feel that (SV) has adjusted to it?

(Foll low same procedure as $\$ 66$ ).

$0=$ no/not quite $1=$ somewhat 2 = pretty well $3=$ very well

68. Have you found anything positive and good for you in this experience with 's stroke? RECORD VERBATIM RESPONSE.

69. What advice would you give to someone whose spouse, parent, relative, or friend has just had a stroke?

70. Is there anything else you would like to add that would be helpful to me in understanding the impact of the stroke(s) on your I ife and well-being?

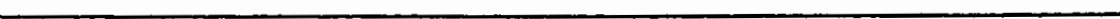


Stroke Study Time 4 Telephone Interview

Thank you very much for your time and you willingness to share your experience. The information you have given is extremely helpful.

There are some other questions which you filled out yourself in the previous interviews. Since people have found them are easier to do themselves than to answer over the phone, I'II send them in the mail for you to fill out and return to me. l'll send a stamped, self-addressed envelope for the ir return, and would appreciate your doing them as soon as possible after you receive them. Thanks again for participating in this study. Cood-bye.

TIME INTERVIEW ENDS (write on front page aiso).

71. Respondent sounded:

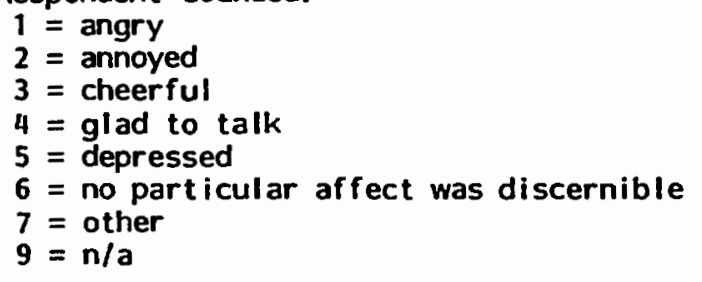

72. Additional comments about the interview:

73. OUTCOME MEASURES: LOT, CES-D. IPWB, *BARTHEL INDEX, *CBS. *PBC (* OAIT IF SV IS DECEASED)

1 = Outcomes completed by self-report.

2 = Outcome measures clarified over phone.

3 = Outcome measures needed to be done over phone. (INDICATE REASON) 
The following packet has some questions about how people feel at different times. There are no right or wrong answers. It is extremely important that every question be answered. Please choose from the available response the answer that best applies to you. Your prompt return of the completed questionnaires in the enclosed envelope is most appreciated. 
I.D. No.

\section{IPWB}

Instructions: Here is a list that describes some of the ways people feel at different times. How often do you feel each of these ways?

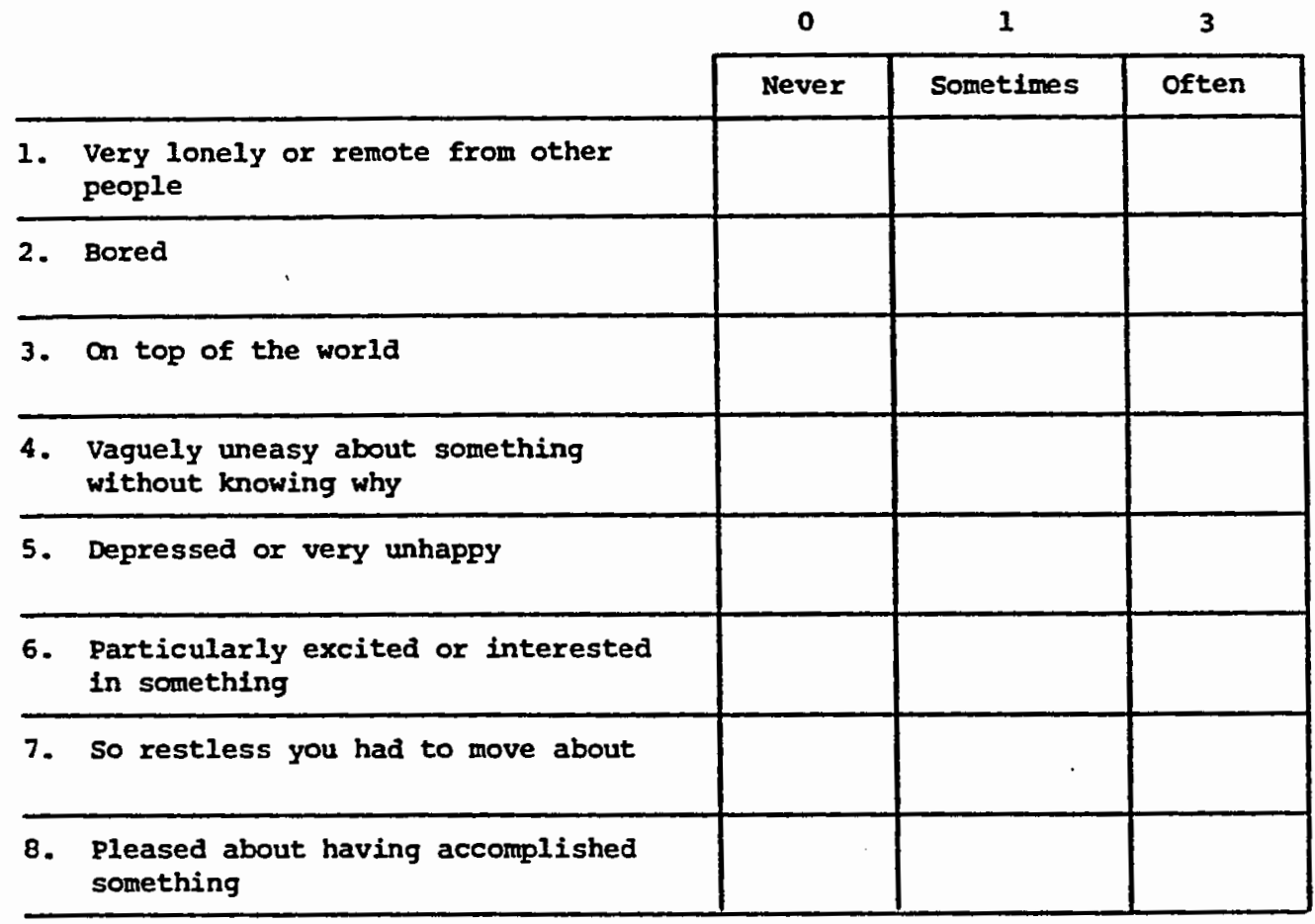


CES-D1

Here 1 a 11st of itews of ways you wight have felt or behaved. Please indicate how often you felt this way during the past week by circling the appropriate response next to each 1 tem.

IN THE PAST WEER, HOW MANY DAYS DID THIS BAPPEN TO YOU?

1. I was bothered by things that usually don't bother me.

2. I did not feel like eating, my appetite vas poor.

3. I felt that I could not shake off the blues even with help from my friends and family.

4. I felt that I was just as good as other people.

5. I had trouble keeping my wind on what $I$ was doing.

6. I felt depressed.

7. I felt that everything I did was an effort.

8. I felt hopeful about the future.

9. I thought my life had been a failure.

10. I felt fearful.

\begin{tabular}{l} 
Rarely \\
or none \\
of the \\
time \\
(Less \\
than 1 \\
day) \\
\hline
\end{tabular}

0

0

0

\begin{tabular}{l} 
Some or \\
a little \\
of the \\
time \\
(1-2 \\
days) \\
\hline
\end{tabular}

1

1

Occastion-

ally or a

moderate

omount

of time

(3-4

days)

2

2

2

0

1

2

0

1

2

0

0

1

1

2

2

0

0

0

1

2
Most or

of

(5-?

days)
3

3

3

3

3

3

3

3 


\section{CES-D2}

11. My sleep was restless.

\begin{tabular}{l} 
Rarely \\
or none \\
of the \\
time \\
(Less \\
than 1 \\
day) \\
\hline
\end{tabular}

\begin{tabular}{l} 
Some or \\
a litele \\
of the \\
time \\
$(1-2$ \\
days) \\
\hline
\end{tabular}

Occasional1y or a

goderate

amount

of time

(3-4

days)

$$
\text { o }
$$

1

1

12. I was happy.

o

13. I talked less than usual.

o

\section{1}

Most or all of the time ( $5-7$

14. I felt lonely.

\section{1} days)

15. People were unfriendly.

16. I enjoyed 11fe.

17. I had crylng spells.

18. I felt sad.

19. I felt that people disliked we.

2

2

2

2

3

3

3

3

2

2

2

2

2

2 "going." 
Please ansuer the rollowing questions about yourselr by c1reling the appropriate aumber after eacb atatemeat. For eacb of tbe statements, indicate the extent of jour agreement by using tbe folloulag scale:

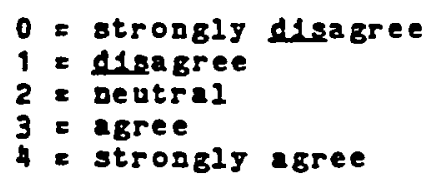


INSTRUCIONS: Please indicete the level of acsivity of the stroike patient in your family by circling the eppropriate number next to each activity.

Barthel Index (Naryland Disability Index)

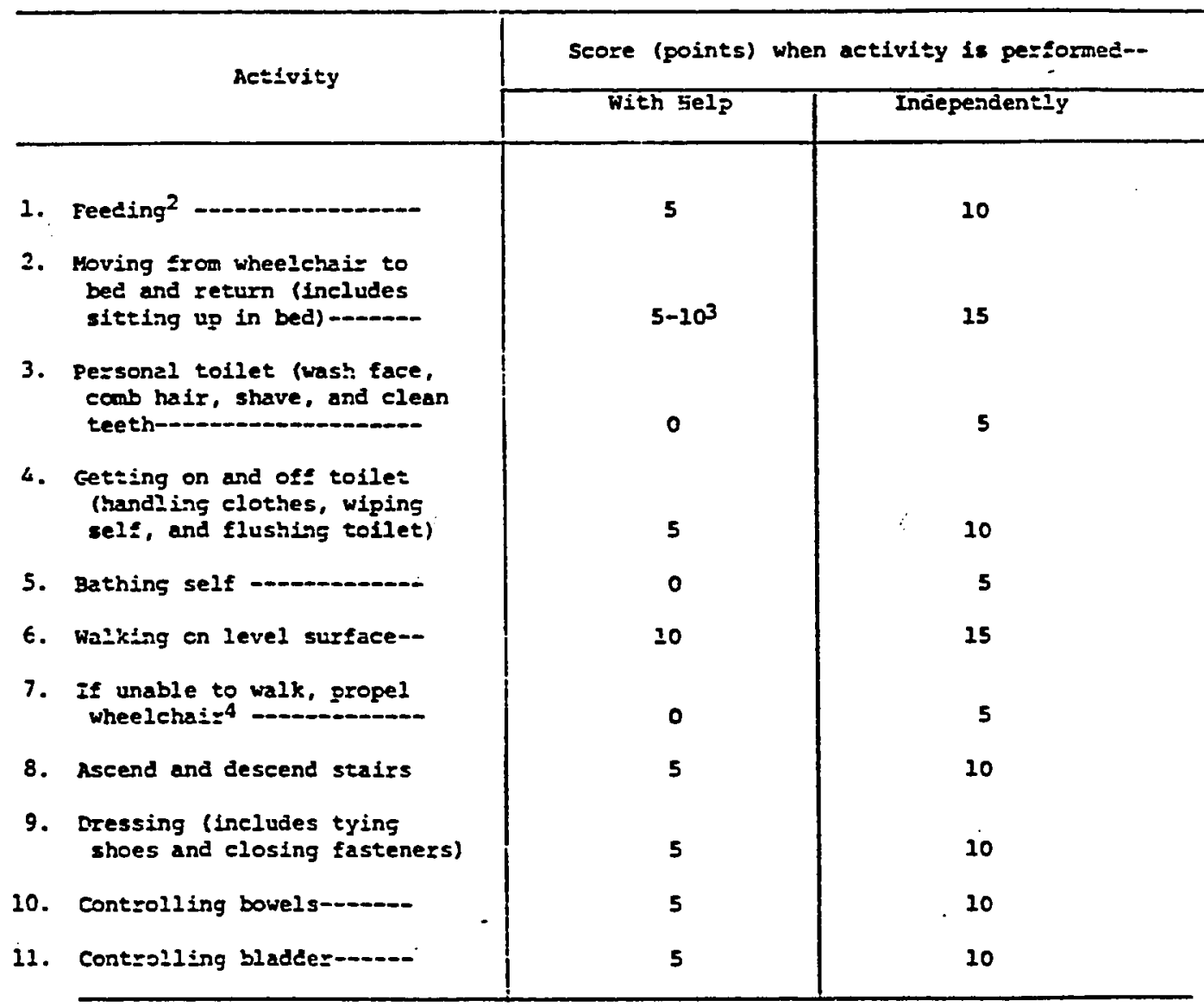

1

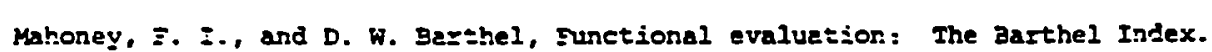
ㄹ. State Mecicel Journal. 14, 6i-65 (1965).

2 If food must be cut ug, the jatient is scored as neecing help.

3

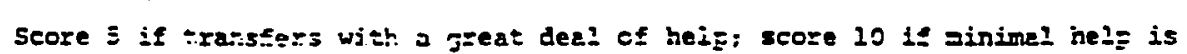
needed.

4 score orly is lineble to walk. 
PBC

Some families and friends have observed personality and behavior changes in a person following a stroke. While other people see the stroke patient as very much the same in personality and behavior as they were before. Please Indicate whether (the stroke patient) behaves or feels like this less than before the stroke. about the same as before the stroke, or more since the stroke. If the stroke patient was never like this before the stroke, the answer would be "about the same".

1. thoughtful (considerate)

2. angry (short-tempered)

3. irritable (easily upset)

4. restless

5. self-centered

6. anxious (fearful, nervous)

7. shows initiative (planning)

8. impulsive (uses poor judgment)

9. laughs

10. cries

11. patient (tolerant)

12. indifferent (lacks initiative)

13. pleasant, easy to get along with

14. suspicious

15. jealous (possessive)

16. confused (perplexed)

17. depressed (sad)

18. happy (cheerful)

19. forgetful (poor memory)

\section{Less}

About the same

More

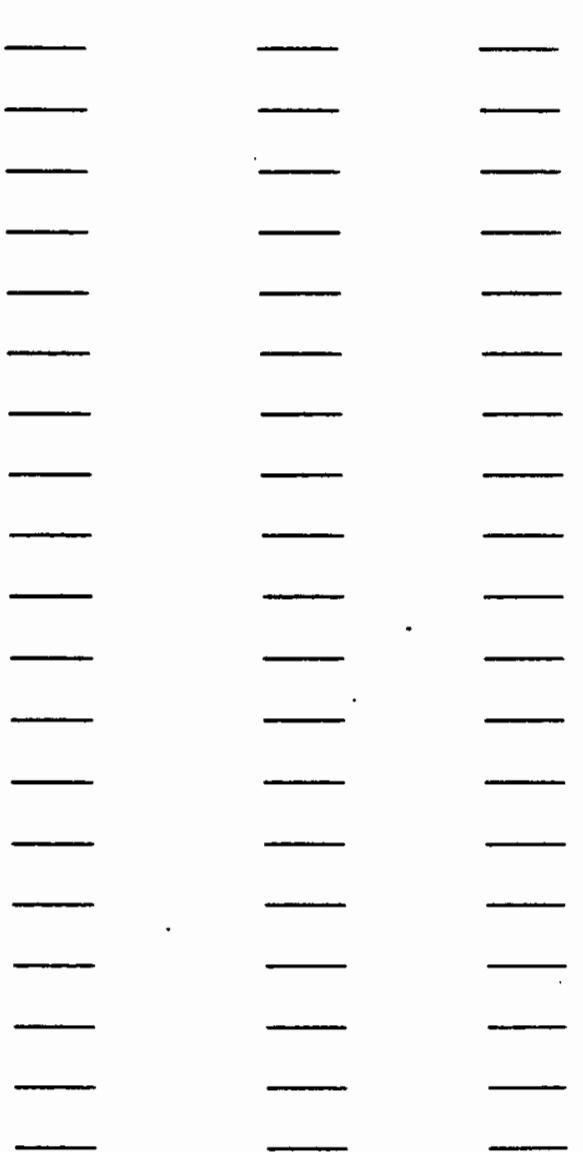


CBS

Here is a list of statements that describe how people sometimes feel when caring for someone who has had a stroke. After reading each statement, put an " $x$ " by the most appropriate word that describes your feelings, from "never to "almost always".

1. I feel that I don't do as much for the stroke patient as I could or should.
Never
Rarely
Sometimes
Often
Aimost always

2. I feel the stroke patient is dependent.

__ Never Rarely ___ Sometimes___ Often ___ Almost always

3. I feel that I am contributing to the well-being of the stroke patient.
Never

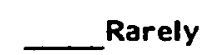
Sometimes
Often
Almost always

4. I feel that the stroke patient makes requests of me that are over and above what he/she needs.

_ Never __ Rarely ___ Sometimes ___ Often __ Almost always

5. Because of my involvement with the stroke patient, I don't have enough time to myself.
Never
Rarely
Sometimes
Often
Almost always

6. I feel resentful of other relatives who could but who do not do things with or for the stroke patient.
Never
Rarely
Sometimes
Often
Almost always

7. I feel stressed between trying to give to the stroke patient as well as to other family responsibilities. job, etc.
Never
Rarely
Sometimes
Often
Almost always

8. I feel that the stroke patient currently affects my relationships with other family and friends in a negative way.
Never
Rarely
Sometimes
Often
Almost always 
9. I feel useful in my interactions with the stroke patient.

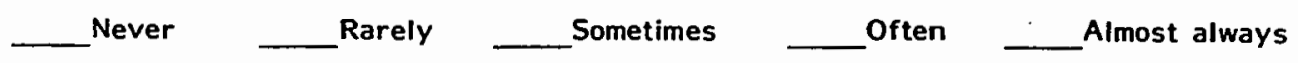

10. I feel that my health has suffered because of my involvenent with the stroke patient.
Never
Rarely
Sometimes
Often
Almost always

11. I feel that my social life has suffered because of my involvement with the stroke patient.

_ Never ___ Rarely ___ Sometimes___ Often __ Almost always

12. I feel that the stroke patient doesn't appreciate what I do for him/her as much as I would like.

Never ___ Rarely ___ Sometimes____ Often ___ Almost always

13. I feel that the stroke patient seems to expect me to take care of him/her as if I were the only one he/she could depend on.

Never Rarely___ Sometimes___ Often Almost always

14. I feel embarrassed over the stroke patient's behavior or condition.

Never ___ Rarely___ Sometimes___ Often __ Almost always 